\title{
BOUNDED LITTLEWOOD IDENTITIES
}

\author{
ERIC M. RAINS AND S. OLE WARNAAR
}

\begin{abstract}
We describe a method, based on the theory of MacdonaldKoornwinder polynomials, for proving bounded Littlewood identities. Our approach provides an alternative to Macdonald's partial fraction technique and results in the first examples of bounded Littlewood identities for Macdonald polynomials. These identities, which take the form of decomposition formulas for Macdonald polynomials of type $(R, S)$ in terms Macdonald polynomials of type A, are $q, t$-analogues of known branching formulas for characters of the symplectic, orthogonal and special orthogonal groups, important in the theory of plane partitions.

As applications of our results we obtain combinatorial formulas for characters of affine Lie algebras, Rogers-Ramanujan identities for such algebras complementing recent results of Griffin et al., and transformation formulas for Kaneko-Macdonald-type hypergeometric series.
\end{abstract}

Keywords: Macdonald-Koornwinder polynomials, Hall-Littlewood polynomials, virtual Koornwinder integrals, characters formulas, Rogers-Ramanujan identities.

\section{Contents}

1. Introduction

2. Macdonald-Koornwinder theory

2.1. Partitions

2.2. Generalised $q$-shifted factorials

2.3. Rogers-Szegö polynomials

2.4. Plethystic notation

2.5. Macdonald polynomials

2.6. Koornwinder polynomials

2.7. Macdonald-Koornwinder polynomials

3. Virtual Koornwinder integrals

4. Bounded Littlewood identities

4.1. Statement of results

4.2. Proofs of Theorems 4.14 .8

5. Applications

2010 Mathematics Subject Classification. 05E05, 05E10, 17B67, 33D67.

Work supported by the National Science Foundation (grant number DMS-1001645) and the Australian Research Council. 
5.1. Character identities for affine Lie algebras 54

5.2. Rogers-Ramanujan identities 63

5.3. Kaneko-Macdonald hypergeometric series

6. Open problems $\quad 80$

Appendix A. The Weyl-Kac formula 83

References 87

\section{INTRODUCTION}

In his 1950 text on group characters [59] D. E. Littlewood presented three identities for Schur functions which can be viewed as reciprocals of the Weyl denominator formulas for the classical groups $\mathrm{B}_{n}, \mathrm{C}_{n}$ and $\mathrm{D}_{n}$. The $\mathrm{B}_{n}$ casewhich earlier appeared in an exercise by Schur [86] —is given by [59, Eq. $(11.9 ; 6)]$

$$
\sum_{\lambda} s_{\lambda}(x)=\prod_{i=1}^{n} \frac{1}{1-x_{i}} \prod_{1 \leqslant i<j \leqslant n} \frac{1}{1-x_{i} x_{j}},
$$

where $s_{\lambda}(x)=s_{\lambda}\left(x_{1}, \ldots, x_{n}\right)$ is a Schur function indexed by the partition $\lambda$. Almost 30 years later, Macdonald [63] proved the following bounded analogue of (1.1)

$$
\sum_{\substack{\lambda \\ \lambda_{1} \leqslant m}} s_{\lambda}(x)=\frac{\operatorname{det}_{1 \leqslant i, j \leqslant n}\left(x_{i}^{m+2 n-j}-x_{i}^{j-1}\right)}{\prod_{i=1}^{n}\left(x_{i}-1\right) \prod_{1 \leqslant i<j \leqslant n}\left(x_{i}-x_{j}\right)\left(x_{i} x_{j}-1\right)},
$$

for $m$ a nonnegative integer, and observed that it implied MacMahon's famous conjecture for the generating function of symmetric plane partitions. By reading off the 'sequence of diagonal slices' - an idea of Okounkov and Reshetikhin [74] more recent than [63] - it immediately follows that the generating function for symmetric plane partitions $\pi$ contained in a box $\mathrm{B}(n, n, m)$ of size $n \times n \times m$ is given by

$$
\sum_{\substack{\lambda \\ \lambda_{1} \leqslant m}} s_{\lambda}\left(q, q^{3}, \ldots, q^{2 n-1}\right) .
$$

Hence MacMahon's formula [68] (see [46] for a recent survey)

$$
\sum_{\pi \in \mathrm{B}(n, n, m)} q^{|\pi|}=\prod_{i=1}^{n} \frac{1-q^{m+2 i-1}}{1-q^{2 i-1}} \prod_{1 \leqslant i<j \leqslant n} \frac{1-q^{2(m+i+j-1)}}{1-q^{2(i+j-1)}}
$$

should follow from the evaluation of the determinant in (1.2) when $x_{i}=q^{2 i-1}$ for $1 \leqslant i \leqslant n$. Since this determinant is essentially a character of the irreducible $\mathrm{SO}(2 n+1, \mathbb{C})$-module of highest weight $m \Lambda_{n}$, the required determinant evaluation corresponds to the $q$-dimension of this module, and follows from the 
Weyl character formula. To prove (1.2) - a $\mathrm{SO}(2 n+1, \mathbb{C})$ to $\mathrm{GL}(n, \mathbb{C})$ branching formula-Macdonald developed a partial fraction method, resulting in a more general $t$-analogue for Hall-Littlewood polynomials.

Since the work of Littlewood and Macdonald many additional Littlewood identities have been discovered and applied to problems in combinatorics, representation theory and $q$-series. Examples include the enumeration of plane partitions and related combinatorial objects such as tableaux, tilings and alternating-sign matrices [7, 8, 11, 12, 19, 20, 29, 30, 44, 75, 76, 88, the computation of characters and branching rules for classical groups and affine Lie algebras $[5,35,41,42,45,72$ and the proof of Rogers-Ramanujan and elliptic hypergeometric series identities [31, 34, 37, 47, 79, 88, 96]. Surprisingly, despite the interest in Littlewood identities, $q$, t-analogues of (1.2) and other bounded Littlewood identities for Schur and Hall-Littlewood polynomials have remained elusive. In this paper we present an approach to Littlewood identities based on the theory of Macdonald-Koornwinder polynomials. As a result we obtain the missing $q, t$-analogues, including the following generalisation of Macdonald's $(1.2) 1$.

Theorem 1.1. For $x=\left(x_{1}, \ldots, x_{n}\right)$ and $m$ a nonnegative integer,

$$
\begin{array}{r}
\sum_{\substack{\lambda \\
\lambda_{1} \leqslant m}} P_{\lambda}(x ; q, t) \prod_{\substack{s \in \lambda \\
l^{\prime}(s) \text { even }}} \frac{1-q^{m-a^{\prime}(s)} t^{l^{\prime}(s)}}{1-q^{m-a^{\prime}(s)-1} t^{l^{\prime}(s)+1}} \prod_{\substack{s \in \lambda \\
l(s) \text { even }}} \frac{1-q^{a(s)} t^{l(s)+1}}{1-q^{a(s)+1} t^{l(s)}} \\
=\left(x_{1} \cdots x_{n}\right)^{\frac{m}{2}} P_{\left(\frac{m}{2}\right)^{n}}^{\left(\mathrm{B}_{n}, \mathrm{~B}_{n}\right)}(x ; q, t, t) .
\end{array}
$$

On the left, $a(s), l(s), a^{\prime}(s), l^{\prime}(s)$ are the arm-length, leg-length, arm-colength and leg-colength of the square $s \in \lambda$ and $P_{\lambda}(x ; q, t)$ is a Macdonald polynomial of type A. The (Laurent) polynomial $P_{\left(\frac{m}{2}\right)^{n}}^{\left(\mathrm{B}_{n}, \mathrm{~B}_{n}\right)}(x ; q, t, t)$ on the right is a Macdonald polynomial attached to the pair of root systems $\left(\mathrm{B}_{n}, \mathrm{~B}_{n}\right)$ indexed by the rectangular partition or 'half-partition' $(m / 2, \ldots, m / 2)$.

Our method also leads to alternative proofs as well as further examples of Littlewood identities for the characters of irreducible highest weight modules of affine Lie algebras, first discovered in [5]. In particular our methods shows that such characters arise by taking suitable limits of Hall-Littlewood polynomials of type $R$. For example, for the twisted affine Lie algebra $\mathrm{A}_{2 n}^{(2)}$ we may claim the following result in terms of modified Hall-Littlewood polynomials $P_{\lambda}^{\prime}(x ; t)$ and the large- $r$ limit of the Hall-Littlewood polynomial $P_{\left(\frac{m}{2}\right)^{n}}^{\left(\mathrm{B}_{r}\right)}\left(x ; t, t_{2}\right)$.

\footnotetext{
${ }^{1}$ An alternative generalisation in terms of $P_{\left(\frac{m}{2}\right)^{n}}^{\left(\mathrm{B}_{n}, \mathrm{C}_{n}\right)}$ is given in Theorem 4.6 .
} 
Theorem 1.2. Let $\alpha_{0}, \ldots, \alpha_{n}$ and $\Lambda_{0}, \ldots, \Lambda_{n}$ be the simple roots and fundamental weights of $\mathrm{A}_{2 n}^{(2)}$, and $\delta=2 \alpha_{0}+\cdots+2 \alpha_{n-1}+\alpha_{n}$ the null root. Set

$$
t=\mathrm{e}^{-\delta} \quad \text { and } \quad x_{i}=\mathrm{e}^{-\alpha_{i}-\cdots-\alpha_{n-1}-\alpha_{n} / 2},
$$

and let $\operatorname{ch} V(\Lambda)$ denote the character of the integrable highest-weight module $V(\Lambda)$ of highest weight $\Lambda$. Then, for $m$ a positive integer,

$$
\begin{aligned}
\mathrm{e}^{-m \Lambda_{0}} \operatorname{ch} V\left(m \Lambda_{0}\right) & =\lim _{\substack{N \rightarrow \infty\\
}} t^{\frac{1}{2} m n N^{2}} P_{\left(\frac{m}{2}\right)^{2 n N}}^{\left(\mathrm{B}_{2 n N}\right)}\left(t^{1 / 2} X ; t, 0\right) \\
& =\sum_{\substack{\lambda \\
\lambda_{1} \leqslant m}} t^{|\lambda| / 2} P_{\lambda}^{\prime}\left(x_{1}^{ \pm}, \ldots, x_{n}^{ \pm} ; t\right),
\end{aligned}
$$

where $X=X_{N}(x ; t)$ is the alphabet

$$
X=\left(x_{1}^{ \pm}, t x_{1}^{ \pm}, \ldots, t^{N-1} x_{1}^{ \pm}, \ldots \ldots, x_{n}^{ \pm}, t x_{n}^{ \pm}, \ldots, t^{N-1} x_{n}^{ \pm}\right)
$$

and $\left(\ldots, a x_{i}^{ \pm}, \ldots\right):=\left(\ldots, a x_{i}, a x_{i}^{-1}, \ldots\right)$.

As shown by Griffin et al. [31, character formulas such as (1.4) imply Rogers-Ramanujan identities through specialisation. Following their approach, we obtain several new examples of Rogers-Ramanujan identities labelled by affine Lie algebras. For example, from (1.4) we obtain the following new identity, where $P_{\lambda}(x ; t)$ is a Hall-Littlewood polynomial, $\theta(x ; q)$ a modified theta function and $(q ; q)_{\infty}$ a $q$-shifted factorial.

Theorem $1.3\left(\mathrm{~A}_{2 n}^{(2)}\right.$ Rogers-Ramanujan identity). For $m, n$ positive integers let $\kappa=m+2 n+1$. Then

$$
\begin{aligned}
& \sum_{\substack{\lambda \\
\lambda_{1} \leqslant m}} q^{|\lambda| / 2} P_{\lambda}\left(1, q, q^{2}, \ldots ; q^{2 n}\right) \\
& \quad=\frac{\left(q^{\kappa} ; q^{\kappa}\right)_{\infty}^{n-1}\left(q^{\kappa / 2} ; q^{\kappa / 2}\right)_{\infty}}{(q ; q)_{\infty}^{n-1}\left(q^{1 / 2} ; q^{1 / 2}\right)_{\infty}} \prod_{i=1}^{n} \theta\left(q^{i} ; q^{\kappa / 2}\right) \prod_{1 \leqslant i<j \leqslant n} \theta\left(q^{j-i} ; q^{\kappa}\right) \theta\left(q^{i+j} ; q^{\kappa}\right) .
\end{aligned}
$$

As a final application we show that the bounded Littlewood identities for Macdonald polynomials imply transformation formulas for multiple basic hypergeometric series of Kaneko-Macdonald type.

The remainder of this paper is organised as follows. In the next section we review some standard material from Macdonald-Koornwinder theory. This includes a discussion of ordinary (or type A) Macdonald polynomials, Koornwinder polynomials and their lifted and virtual analogues, generalised Macdonald polynomials of type $(R, S)$, Hall-Littlewood polynomials and Rogers-Szegö polynomials. 
In Section 3 we consider two functionals on the ring of symmetric function known as virtual Koornwinder integrals. We review a number of earlier results for virtual Koornwinder integrals and prove several new integral evaluations.

In Section 4 we present our approach to bounded Littlewood identities. The key idea is to show that each bounded Littlewood identity is equivalent to the closed-form evaluation of a virtual Koornwinder integral. We apply our method to prove several new bounded Littlewood identities, including Theorem 1.1 and a $q, t$-analogue of the well-known Désarménien-Proctor-Stembridge determinant, see Theorem 4.1 below.

Section 5 contains applications of the results of Section 4 . First we show how bounded Littlewood identities give rise to combinatorial formulas for characters of affine Lie algebras, such as Theorem 1.2. This provides an alternative to the recent approach of Bartlett and the second author based on the $\mathrm{C}_{n}$ Bailey lemma [5]. As a second application we follow recent ideas of Griffin et al. 31 and apply the combinatorial character identities to prove new Rogers-Ramanujan identities for the affine Lie algebras $\mathrm{B}_{n}^{(1)}, \mathrm{A}_{2 n-1}^{(2)}, \mathrm{A}_{2 n}^{(2)}$ and $\mathrm{D}_{n+1}^{(2)}$. The Rogers-Ramanujan identity for $\mathrm{B}_{n}^{(1)}$, given in Theorem 5.12 and Remark 5.13, generalise Bressoud's even modulus analogues of the celebrated Andrews-Gordon identities. Finally, by appropriately specialising bounded Littlewood identities for Macdonald polynomials, we prove new transformation formulas for multivariable basic hypergeometric series of Kaneko-Macdonaldtype [39,67].

We conclude with an appendix containing some technical lemmas pertaining to the Weyl-Kac formula.

Acknowledgements. We thank Michael Schlosser, Hjalmar Rosengren and Jasper Stokman for helpful discussions on hypergeometric function, Macdonald identities and Macdonald-Koornwinder polynomials. We thank Richard Stanley for pointing out the paper [86] by Schur.

\section{MACDOnALD-KoORNWINDER THEORY}

\subsection{Partitions.}

A partition $\lambda=\left(\lambda_{1}, \lambda_{2}, \ldots\right)$ is a weakly decreasing sequence of nonnegative integers such that only finitely-many $\lambda_{i}$ are positive. The positive $\lambda_{i}$ are called the parts of $\lambda$, and the number of parts, denoted $l(\lambda)$, is called the length of $\lambda$. As is customary, we often ignore the tail of zeros of a partition. If $|\lambda|:=\sum_{i} \lambda_{i}=n$ we say that $\lambda$ is a partition of $n$. The unique partition of 0 is denoted by 0 . As usual we identify a partition with its Young diagrama collection of left-aligned rows of squares such that the $i$ th row contains $\lambda_{i}$ squares. For example, the partition $(5,3,3,1)$ corresponds to 


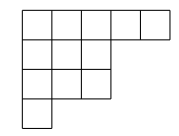

The conjugate partition $\lambda^{\prime}$ is obtained from $\lambda$ by reflection in the main diagonal, so that the parts of $\lambda^{\prime}$ are given by the columns of $\lambda$. Given a partition $\lambda$, the multiplicity $m_{i}(\lambda)=\lambda_{i}^{\prime}-\lambda_{i+1}^{\prime}$ counts the number of parts of size $i$. If $\lambda$ is a rectangular partition consisting of $m$ rows and $n$ columns we write $\lambda=m^{n}$. If $\lambda$ is a partition of length at most $n$ we also write $\lambda+m^{n}$ for $\left(m+\lambda_{1}, \ldots, m+\lambda_{n}\right)$. Finally, the number of even/odd parts of $\lambda$ will be denoted by even $(\lambda) / \operatorname{odd}(\lambda)$.

For two partitions $\lambda, \mu$ we write $\mu \subset \lambda$ if $\mu$ is contained in $\lambda$, i.e., if $\mu_{i} \leqslant \lambda_{i}$ for all $i \geqslant 1$. In this case the set-theoretic difference between $\lambda$ and $\mu$ is called a skew shape. To avoid a notational clash with partition complementation to be defined shortly, we write this difference as $\lambda / \mu$ instead of the the more common $\lambda-\mu$. For example, the skew shape $(5,3,3,1) /(3,3,1)$ is given by

A skew shape $\lambda / \mu$ containing at most one square in each column, as in the above example, is referred to as a horizontal strip. Analogously, a vertical strip is a skew diagram with at most one square in each row. If $\lambda \subset m^{n}$ we write the complement of $\lambda$ with respect to $m^{n}$ as $m^{n}-\lambda$, that is, $m^{n}-\lambda=$ $\left(m-\lambda_{n}, \ldots, m-\lambda_{2}, m-\lambda_{1}\right)$. For example, the complement of $(3,2)$ with respect to $\left(4^{3}\right)$ is $(4,2,1)$.

The dominance order on the set of partitions is defined as follows: $\lambda \geqslant \mu$ if $\lambda_{1}+\cdots+\lambda_{k} \geqslant \mu_{1}+\cdots+\mu_{k}$ for all $k \geqslant 1$. Note that unlike [64] we do not assume $|\lambda|=|\mu|$. If $\lambda \geqslant \mu$ and $\lambda \neq \mu$ we write $\lambda>\mu$.

The arm-length, arm-colength, leg-length and leg-colength of the square $s=(i, j) \in \lambda$ are given by

$$
\begin{aligned}
a(s) & =a_{\lambda}(s)=\lambda_{i}-j, & a^{\prime}(s) & =a_{\lambda}^{\prime}(s)=j-1, \\
l(s) & =l_{\lambda}(s)=\lambda_{j}^{\prime}-i, & l^{\prime}(s) & =l_{\lambda}^{\prime}(s)=i-1,
\end{aligned}
$$

and the hook-length of $s$ is $h(s)=a(s)+l(s)+1$. As usual, the statistic $n(\lambda)$ is given by

$$
n(\lambda):=\sum_{s \in \lambda} l^{\prime}(s)=\sum_{i \geqslant 1}(i-1) \lambda_{i}=\sum_{i \geqslant 1}\left(\begin{array}{c}
\lambda_{i}^{\prime} \\
2
\end{array}\right) .
$$

Finally, we say that $\lambda=\left(\lambda_{1}, \ldots, \lambda_{n}\right)$ is a half-partition if $\lambda_{1} \geqslant \lambda_{2} \geqslant \cdots \geqslant$ $\lambda_{n}>0$ and all $\lambda_{i}$ are half-integers. We sometimes write this as $\lambda=\mu+(1 / 2)^{n}$ with $\mu$ a partition of length at most $n$. Conversely, if $\lambda=\mu+(1 / 2)^{n}$ we also write $\mu=\lambda-(1 / 2)^{n}$. The length of a half-partition $\lambda=\left(\lambda_{1}, \ldots, \lambda_{n}\right)$ is by definition $n$, and $m_{i}(\lambda)$ for $i$ a positive half-integer is the multiplicity 
of parts of size $i$. We use half-partitions to generalise our earlier notion of complementation so that $m^{n}-\lambda$ makes sense for $m$ an integer or half-integer and $\lambda \subset m^{n}$ a partition (of length at most $n$ ) or half-partition (of length $n$ ). For example, $4^{3}-(5 / 2,3 / 2,3 / 2)=(5 / 2,5 / 2,3 / 2)$ and $(7 / 2)^{3}-(5 / 2,3 / 2,3 / 2)=$ $(2,2,1)$. We also extend the dominance order to the set of half-partitions in the obvious way. However, partitions and half-partitions are by definition incomparable.

\subsection{Generalised $q$-shifted factorials.}

Let

$$
(z ; q)_{\infty}:=\prod_{i \geqslant 0}\left(1-z q^{i}\right) \quad \text { and } \quad(z ; q)_{n}:=\frac{(z ; q)_{\infty}}{\left(z q^{n} ; q\right)_{\infty}}
$$

be the standard $q$-shifted factorials [27]. In this paper we will mostly view $q$-series as formal power series, but occasionally we require $q$ to be a complex variable such that $|q|<1$. The modified theta function is defined as

$$
\theta(z ; q):=(z ; q)_{\infty}(q / z ; q)_{\infty}=\frac{1}{(q ; q)_{\infty}} \sum_{k \in \mathbb{Z}} z^{k} q^{\left(\begin{array}{c}
k \\
2
\end{array}\right)} \quad \text { for } z \neq 0,
$$

where the equality between the product and the sum is known as the Jacobi triple product identity. We also need more general $q$-shifted factorials indexed by partitions:

$$
\begin{aligned}
(z ; q, t)_{\lambda} & :=\prod_{s \in \lambda}\left(1-z q^{a^{\prime}(s)} t^{-l^{\prime}(s)}\right)=\prod_{i=1}^{n}\left(z t^{1-i} ; q\right)_{\lambda_{i}} \\
C_{\lambda}^{-}(z ; q, t) & :=\prod_{s \in \lambda}\left(1-z q^{a(s)} t^{l(s)}\right) \\
& =\prod_{i=1}^{n}\left(z t^{n-i} ; q\right)_{\lambda_{i}} \prod_{1 \leqslant i<j \leqslant n} \frac{\left(z t^{j-i-1} ; q\right)_{\lambda_{i}-\lambda_{j}}}{\left(z t^{j-i} ; q\right)_{\lambda_{i}-\lambda_{j}}} .
\end{aligned}
$$

The choice of $n$ on the right is irrelevant as long as $n \geqslant l(\lambda)$. We note that $(a ; q, t)_{\lambda}$ is sometimes denoted as $C_{\lambda}^{0}(a ; q, t)$, see e.g., [81], and that $C_{\lambda}^{-}(t ; q, t)=$ $c_{\lambda}(q, t)$ and $C_{\lambda}^{-}(q ; q, t)=c_{\lambda}^{\prime}(q, t)$, with $c_{\lambda}$ and $c_{\lambda}^{\prime}$ the hook-length polynomials of Macdonald [64, page 352]. In particular, $C_{\lambda}^{-}(q ; q, q)=c_{\lambda}(q, q)=c_{\lambda}^{\prime}(q, q)=$ $H_{\lambda}(q)$ with $H_{\lambda}(q)=\prod_{s \in \lambda}\left(1-q^{h(s)}\right)$ the classical hook-length polynomial. For $s \in \lambda$ let

$$
b_{\lambda}(s ; q, t):=\frac{1-q^{a_{\lambda}(s)} t^{l_{\lambda}(s)+1}}{1-q^{a_{\lambda}(s)+1} t^{l_{\lambda}(s)}} .
$$

Then

$$
b_{\lambda}(q, t):=\frac{c_{\lambda}(q, t)}{c_{\lambda}^{\prime}(q, t)}=\prod_{s \in \lambda} b_{\lambda}(s ; q, t) .
$$


For each of the $q$-shifted factorials as well as the theta function we use condensed notation, such as

$$
\left(z_{1}, \ldots, z_{k} ; q, t\right)_{\lambda}=\left(z_{1} ; q, t\right)_{\lambda} \cdots\left(z_{k} ; q, t\right)_{\lambda} .
$$

It is an elementary exercise to verify the following identities, which will be used throughout the paper:

$$
\begin{aligned}
(a ; q, t)_{\lambda^{\prime}} & =(-a)^{|\lambda|} q^{n(\lambda)} t^{-n\left(\lambda^{\prime}\right)}\left(a^{-1} ; t, q\right)_{\lambda} \\
C_{\lambda^{\prime}}^{-}(a ; q, t) & =C_{\lambda}^{-}(a ; t, q)
\end{aligned}
$$

and

$$
\begin{aligned}
(a ; q, t)_{2 \lambda} & =\left(a, a q ; q^{2}, t\right)_{\lambda} \\
C_{2 \lambda}^{-}(a ; q, t) & =C_{\lambda}^{-}\left(a, a q ; q^{2}, t\right)
\end{aligned}
$$

for $2 \lambda:=\left(2 \lambda_{1}, 2 \lambda_{2}, \ldots\right)$, and

$$
\begin{aligned}
(a ; q, t)_{m^{n-\lambda}} & =\left(-q^{1-m} t^{n-1} / a\right)^{|\lambda|} q^{n\left(\lambda^{\prime}\right)} t^{-n(\lambda)} \frac{(a ; q, t)_{m^{n}}}{\left(q^{1-m} t^{n-1} / a ; q, t\right)_{\lambda}} \\
C_{m^{n}-\lambda}^{-}(a ; q, t) & =\left(-q^{1-m} / a\right)^{|\lambda|} q^{n\left(\lambda^{\prime}\right)} t^{-n(\lambda)} \frac{\left(a t^{n-1} ; q, t\right)_{m^{n}} C_{\lambda}^{-}(a ; q, t)}{\left(a t^{n-1}, q^{1-m} / a ; q, t\right)_{\lambda}} .
\end{aligned}
$$

\subsection{Rogers-Szegö polynomials.}

For integers $k, n$ such that $0 \leqslant k \leqslant n$ let

$$
\left[\begin{array}{l}
n \\
k
\end{array}\right]_{q}:=\frac{(q ; q)_{n}}{(q ; q)_{k}(q ; q)_{n-k}}
$$

be a $q$-binomial coefficient. Then the Rogers-Szegő polynomials $H_{m}(z ; q)$ are defined as [91].

$$
H_{m}(z ; q):=\sum_{k=0}^{m} z^{k}\left[\begin{array}{c}
m \\
k
\end{array}\right]_{q},
$$

for $m$ a nonnegative integer. They satisfy the three-term recurrence

$$
H_{m+1}(z ; q)=(1+z) H_{m}(z ; q)-\left(1-q^{m}\right) z H_{m-1}(z ; q)
$$

with initial conditions $H_{-1}=0, H_{0}=1$, and are orthogonal on the unit circle with respect to the weight function

$$
w(z ; q)=\left|\left(z q^{1 / 2} ; q\right)_{\infty}\right|^{2} \quad(0<q<1) .
$$

The Rogers-Szegő polynomials are closely related to symmetric functions and may be expressed in terms of Schur functions as

$$
H_{m}(z ; q)=(q)_{m} \sum_{\lambda \vdash m} \frac{q^{n(\lambda)}}{H_{\lambda}(q)} s_{\lambda}(1, z),
$$

with $H_{\lambda}(q)$ the hook-length polynomial. 
We require two generalisations of the Rogers-Szegő polynomials to polynomials indexed by partitions. First,

$$
\begin{aligned}
h_{\lambda}^{(m)}(a, b ; q) & :=\prod_{\substack{i=1 \\
i \text { odd }}}^{m-1}(-a)^{m_{i}(\lambda)} H_{m_{i}(\lambda)}(b / a ; q) \prod_{\substack{i=1 \\
i \text { even }}}^{m-1} H_{m_{i}(\lambda)}(a b ; q) \\
& =(-a)^{\operatorname{odd}(\lambda)} \prod_{\substack{i=1 \\
i \text { odd }}}^{m-1} H_{m_{i}(\lambda)}(b / a ; q) \prod_{\substack{i=1 \\
i \text { even }}}^{m-1} H_{m_{i}(\lambda)}(a b ; q),
\end{aligned}
$$

where $m$ is a nonnegative integer. Compared to earlier definitions in $[5,96]$ the parameters $a$ and $b$ have been replaced by their negatives. Since $H_{m}$ is a reciprocal polynomial it follows that $h_{\lambda}^{(m)}(a, b ; q)$ is symmetric in $a$ and $b$ :

$$
h_{\lambda}^{(m)}(a, b ; q)=h_{\lambda}^{(m)}(b, a ; q) .
$$

Since $m_{i}(\lambda)=0$ for $i>\lambda_{1}$, the upper bound on the products over $i$ in (2.8) may be dropped if $m \geqslant \lambda_{1}+1$. For such $m$ we simply write $h_{\lambda}(a, b ; q)$. That is,

$$
h_{\lambda}(a, b ; q)=\prod_{\substack{i \geqslant 1 \\ i \text { odd }}}(-a)^{m_{i}(\lambda)} H_{m_{i}(\lambda)}(b / a ; q) \prod_{\substack{i \geqslant 1 \\ i \text { even }}} H_{m_{i}(\lambda)}(a b ; q) .
$$

We further define

$$
h_{\lambda}^{(m)}(a ; t):=h_{\lambda}^{(m)}(a,-1 ; t)=\prod_{i=1}^{m-1} H_{m_{i}(\lambda)}(-a ; t) .
$$

Since 96

$$
\begin{aligned}
H_{m}(0 ; t) & =1 \\
H_{m}(-1 ; t) & = \begin{cases}\left(t ; t^{2}\right)_{m / 2} & m \text { even } \\
0 & m \text { odd }\end{cases} \\
H_{m}(-t ; t) & =\left(t ; t^{2}\right)_{\lceil m / 2\rceil} \\
H_{m}\left(t^{1 / 2} ; t\right) & =\left(-t^{1 / 2} ; t^{1 / 2}\right)_{m},
\end{aligned}
$$

there are a number of choices for $a$ and $b$ for which (2.8) and (2.10) completely factor. This will be important later when considering character formulas for affine Lie algebras.

\subsection{Plethystic notation.}

Let $\mathfrak{S}_{n}$ be the symmetric group on $n$ letters, $\Lambda_{n}=\mathbb{F}\left[x_{1}, \ldots, x_{n}\right]^{\mathfrak{S}_{n}}$ the ring of symmetric functions in the alphabet $x_{1}, \ldots, x_{n}$ with coefficients in $\mathbb{F}$, and $\Lambda$ the corresponding ring of symmetric functions in countably many variables, 
see [64]. We will mostly consider $\mathbb{F}=\mathbb{Q}(q, t)$ or $\mathbb{F}=\mathbb{Q}\left(q, t, t_{0}, t_{1}, t_{2}, t_{3}\right)$ or variations thereof.

To facilitate computations in $\Lambda$ we frequently employ plethystic or $\lambda$-ring notation [33, 48]. This is most-easily described in terms of the Newton power sums

$$
p_{r}(x):=x_{1}^{r}+x_{2}^{r}+\cdots, \quad r \geqslant 1,
$$

with generating function

$$
\Psi_{z}(x):=\sum_{r=1}^{\infty} z^{r-1} p_{r}(x)=\prod_{i \geqslant 1} \frac{x_{i}}{1-z x_{i}} .
$$

The $p_{r}$ form an algebraic basis of $\Lambda$, that is, $\Lambda=\mathbb{F}\left[p_{1}, p_{2}, \ldots\right]$.

If $x=\left(x_{1}, x_{2}, \ldots\right)$ we additively write $x=x_{1}+x_{2}+\cdots$ and to indicate the latter notation we use plethystic brackets:

$$
f(x)=f\left(x_{1}, x_{2}, \ldots\right)=f\left[x_{1}+x_{2}+\cdots\right]=f[x], \quad f \in \Lambda .
$$

The sum, difference and Cartesian product of two alphabets $x$ and $y$ are then defined as

$$
p_{r}[x+y]:=p_{r}[x]+p_{r}[y], \quad p_{r}[x-y]:=p_{r}[x]-p_{r}[y], \quad p_{r}[x y]=p_{r}[x] p_{r}[y] .
$$

In particular, if $x$ is the empty alphabet then $p_{r}[-y]=-p_{r}[y]$ and if $y$ contains only a single letter, say $y_{1}$, then $p_{r}\left[-y_{1}\right]=-p_{r}\left[y_{1}\right]=-y_{1}^{r}$. This should not be confused with $p_{r}\left(-y_{1}\right)=\left(-y_{1}\right)^{r}$. Occasionally we need to also use ordinary minus signs in plethystic notation. To distinguish this from a plethystic minus sign we denote by the $\varepsilon$ the alphabet consisting of the single letter -1 , so that

$$
f(-x)=f\left(-x_{1},-x_{2}, \ldots\right)=f\left[\varepsilon x_{1}+\varepsilon x_{2}+\cdots\right]=f[\varepsilon x] .
$$

Hence

$$
p_{r}[\varepsilon x]=(-1)^{r} p_{r}[x], \quad p_{r}[-\varepsilon x]=(-1)^{r-1} p_{r}[x]
$$

and

$$
f[x+\varepsilon]=f\left(-1, x_{1}, x_{2}, \ldots\right) .
$$

For indeterminates $a, b, t$ and $f \in \Lambda$ we further define $f[(a-b) /(1-t)]$ by

$$
p_{r}\left[\frac{a-b}{1-t}\right]=\frac{a^{r}-b^{r}}{1-t^{r}}
$$

and note that $(a-b) /(1-t)$ may be viewed as the difference between the alphabets $a\left(1+t+t^{2}+\cdots\right)$ and $b\left(1+t+t^{2}+\cdots\right)$, where $a\left(1+t+t^{2}+\cdots\right)$ is the Cartesian product of the single-letter alphabet $a$ and the infinite alphabet $1+t+t^{2}+\cdots$. Alternatively, $(a-b) /(1-t)$ may be interpreted as the the 
Cartesian product of $a-b$ and $1+t+t^{2}+\cdots$. We can of course combine (2.12) and (2.13), and for example

$$
p_{r}\left[x+\frac{a-b}{1-t}\right]=p_{r}[x]+p_{r}\left[\frac{a-b}{1-t}\right] .
$$

For $r \geqslant 0$, the complete symmetric functions are defined by

$$
h_{r}(x):=\sum_{1 \leqslant i_{1} \leqslant i_{2} \leqslant \cdots \leqslant i_{r}} x_{i_{1}} x_{i_{2}} \cdots x_{i_{r}}
$$

and admit the simple generating function

$$
\sigma_{z}(x):=\sum_{r \geqslant 0} z^{r} h_{r}(x)=\prod_{i \geqslant 1} \frac{1}{1-z x_{i}} .
$$

Since $\Psi_{z}(x)=\frac{\mathrm{d}}{\mathrm{d} z} \log \sigma_{z}(x)$ it follows that

$$
\begin{aligned}
\sigma_{1}[x+y] & =\sigma_{1}[x] \sigma_{1}[y]=\prod_{i \geqslant 1} \frac{1}{\left(1-x_{i}\right)\left(1-y_{i}\right)} \\
\sigma_{1}[x-y] & =\frac{\sigma_{1}[x]}{\sigma_{1}[y]}=\prod_{i \geqslant 1} \frac{1-y_{i}}{1-x_{i}} \\
\sigma_{1}\left[\frac{a-b}{1-t}\right] & =\prod_{k \geqslant 0} \frac{\sigma_{1}\left[a t^{k}\right]}{\sigma_{1}\left[b t^{k}\right]}=\frac{(b ; t)_{\infty}}{(a ; t)_{\infty}}
\end{aligned}
$$

These three formulæ allow various infinite products to be expressed in terms of symmetric functions.

Finally, for $\mathbb{F}=\mathbb{Q}(q, t), \omega_{q, t}$ is the $\mathbb{F}$-algebra endomorphism of $\Lambda$ given by [64, page 312$]$

$$
\omega_{q, t} p_{r}=(-1)^{r-1} \frac{1-q^{r}}{1-t^{r}} p_{r} .
$$

Note that $\omega_{t, q}=\omega_{q, t}^{-1}$, and that plethystically

$$
\omega_{q, t} f(x)=f\left(\left[-\varepsilon \frac{1-q}{1-t} x\right]\right), \quad f \in \Lambda .
$$

\subsection{Macdonald polynomials.}

Let $\mathbb{F}=\mathbb{Q}(q, t)$. The power sums $p_{\lambda}:=\prod_{i=1}^{l(\lambda)} p_{\lambda_{i}}$ may be used to define a $q, t$-Hall scalar product on $\Lambda$ as 64

$$
\left\langle p_{\lambda}, p_{\mu}\right\rangle_{q, t}:=\delta_{\lambda \mu} z_{\lambda} \prod_{i=1}^{n} \frac{1-q^{\lambda_{i}}}{1-t^{\lambda_{i}}}
$$


where $z_{\lambda}=\prod_{i \geqslant 1} m_{i}(\lambda) ! i^{m_{i}(\lambda)}$. The Macdonald polynomials $P_{\lambda}(q, t)=P_{\lambda}(x ; q, t)$ are the unique family of symmetric functions such that 64 ]

$$
P_{\lambda}(q, t)=m_{\lambda}+\sum_{\mu<\lambda} u_{\lambda \mu}(q, t) m_{\mu}
$$

and

$$
\left\langle P_{\lambda}(q, t), P_{\mu}(q, t)\right\rangle_{q, t}=0 \quad \text { if } \quad \lambda \neq \mu .
$$

Here the $m_{\lambda}$ are the monomial symmetric functions, given by

$$
m_{\lambda}(x)=\sum_{\alpha} x^{\alpha}
$$

with $\alpha$ summed over distinct permutations of $\lambda$. It immediately follows that the Macdonald polynomials form a basis of $\Lambda$. When $l(\lambda)>n, P_{\lambda}\left(x_{1}, \ldots, x_{n} ; q, t\right)=$ 0 and $\left\{P_{\lambda}\left(x_{1}, \ldots, x_{n} ; q, t\right)\right\}_{l(\lambda) \leqslant n}$ form a basis of $\Lambda_{n}$.

The skew Macdonald polynomials $P_{\lambda / \mu}(q, t)$ are defined by

$$
\left\langle P_{\lambda / \mu}(q, t), P_{\nu}(q, t)\right\rangle_{q, t}=\left\langle P_{\lambda}(q, t), P_{\mu}(q, t) P_{\nu}(q, t)\right\rangle_{q, t},
$$

and vanish unless $\mu \subset \lambda$. For $q=t$ the Macdonald polynomials simplify to the classical Schur functions: $P_{\lambda / \mu}(t, t)=s_{\lambda / \mu}$.

For later comparison with the Koornwinder and $(R, S)$ Macdonald polynomials we remark that an alternative description of the Macdonald polynomials on $n$ variables is as the unique family of polynomials (2.16) such that 64]

$$
\left\langle P_{\lambda}, P_{\mu}\right\rangle_{q, t}^{\prime}=0 \quad \text { if } \lambda \neq \mu
$$

where, for $|q|,|t|<1,\langle\cdot, \cdot\rangle_{q, t}^{\prime}=0$ is the scalar product on $\mathbb{F}[x]=\mathbb{F}\left[x_{1}, \ldots, x_{n}\right]$ defined by

$$
\langle f, g\rangle_{q, t}^{\prime}:=\frac{1}{n !(2 \pi \mathrm{i})^{n}} \int_{\mathbb{T}^{n}} f(x) g\left(x^{-1}\right) \Delta(x ; q, t) \frac{\mathrm{d} x_{1}}{x_{1}} \cdots \frac{\mathrm{d} x_{n}}{x_{n}} .
$$

Here $f\left(x^{-1}\right)=f\left(x_{1}^{-1}, \ldots, x_{n}^{-1}\right)$ and $\Delta(x ; q, t)$ is the Macdonald density

$$
\Delta(x ; q, t):=\prod_{1 \leqslant i<j \leqslant n} \frac{\left(x_{i} / x_{j}, x_{j} / x_{i} ; q\right)_{\infty}}{\left(t x_{i} / x_{j}, t x_{j} / x_{i} ; q\right)_{\infty}} .
$$

Below we list a number of standard results from Macdonald polynomial theory needed later. First of all, defining a second family of Macdonald polynomials $Q_{\lambda / \mu}(q, t)=Q_{\lambda / \mu}(x ; q, t)$ as

$$
Q_{\lambda / \mu}(q, t):=\frac{b_{\lambda}(q, t)}{b_{\mu}(q, t)} P_{\lambda / \mu}(q, t),
$$

with $b_{\lambda}(q, t)$ given by (2.3) , we have

$$
\omega_{q, t} P_{\lambda}(q, t)=Q_{\lambda^{\prime}}(t, q)
$$


as well as

$$
\left\langle P_{\lambda}(q, t), Q_{\mu}(q, t)\right\rangle_{q, t}=\delta_{\lambda \mu}
$$

This last equation is equivalent to the Cauchy identity

$$
\sum_{\lambda} P_{\lambda}(x ; q, t) Q_{\lambda}(y ; q, t)=\prod_{i, j \geqslant 1} \frac{\left(t x_{i} y_{j} ; q\right)_{\infty}}{\left(x_{i} y_{j} ; q\right)_{\infty}}
$$

which we repeatedly require in the dual form

$$
\sum_{\lambda \subset m^{n}}(-1)^{|\lambda|} P_{\lambda}\left(x_{1}, \ldots, x_{n} ; q, t\right) P_{\lambda^{\prime}}\left(y_{1}, \ldots, y_{m} ; t, q\right)=\prod_{i=1}^{n} \prod_{j=1}^{m}\left(1-x_{i} y_{j}\right) .
$$

We also need the $g$ - and $e$-Pieri rules for Macdonald polynomials expressed in generating function form. First, in the $g$-Pieri case,

$$
P_{\mu}(q, t) \prod_{i \geqslant 1} \frac{\left(a t x_{i} ; q\right)_{\infty}}{\left(a x_{i} ; q\right)_{\infty}}=\sum_{\lambda \supset \mu} a^{|\lambda / \mu|} \varphi_{\lambda / \mu}(q, t) P_{\lambda}(q, t) .
$$

Here the Pieri coefficient $\varphi_{\lambda / \mu}(q, t)=0$ unless $\lambda / \mu$ is a horizontal strip, in which case

$$
\begin{aligned}
\varphi_{\lambda / \mu}(q, t)= & \prod_{1 \leqslant i \leqslant j \leqslant l(\lambda)} \frac{\left(q t^{j-i} ; q\right)_{\lambda_{i}-\lambda_{j}}}{\left(t^{j-i+1} ; q\right)_{\lambda_{i}-\lambda_{j}}} \cdot \frac{\left(q t^{j-i} ; q\right)_{\mu_{i}-\mu_{j+1}}}{\left(t^{j-i+1} ; q\right)_{\mu_{i}-\mu_{j+1}}} \\
& \times \frac{\left(t^{j-i+1} ; q\right)_{\lambda_{i}-\mu_{j}}}{\left(q t^{j-i} ; q\right)_{\lambda_{i}-\mu_{j}}} \cdot \frac{\left(t^{j-i+1} ; q\right)_{\mu_{i}-\lambda_{j+1}}}{\left(q t^{j-i} ; q\right)_{\mu_{i}-\lambda_{j+1}}} .
\end{aligned}
$$

Similarly, the e-Pieri rule is given by

$$
\left.P_{\mu}(x ; q, t) \prod_{i \geqslant 1}\left(1+a x_{i}\right)=\sum_{\lambda \supset \mu} a^{|\lambda / \mu|} \psi_{\lambda / \mu}^{\prime} q, t\right) P_{\lambda}(x ; q, t),
$$

where $\psi_{\lambda / \mu}^{\prime}(q, t)$ is zero unless $\lambda / \mu$ is a vertical strip, in which case [64, page $336]$

$$
\psi_{\lambda / \mu}^{\prime}(q, t)=\prod \frac{1-q^{\mu_{i}-\mu_{j}} t^{j-i-1}}{1-q^{\mu_{i}-\mu_{j}} t^{j-i}} \cdot \frac{1-q^{\lambda_{i}-\lambda_{j}} t^{j-i+1}}{1-q^{\lambda_{i}-\lambda_{j}} t^{j-i}} .
$$

The product in the above is over all $i<j$ such that $\lambda_{i}=\mu_{i}$ and $\lambda_{j}>\mu_{j}$. An alternative expression for $\psi_{\lambda / \mu}^{\prime}(q, t)$ is given by [64, page 340]

$$
\psi_{\lambda / \mu}^{\prime}(q, t)=\prod \frac{b_{\lambda}(s ; q, t)}{b_{\mu}(s ; q, t)}
$$

where the product is over all squares $s=(i, j) \in \mu \subset \lambda$ such that $i<j$, $\mu_{i}=\lambda_{i}$ and $\lambda_{j}^{\prime}>\mu_{j}^{\prime}$. 
For $\lambda$ a partition define

$$
b_{\lambda}^{\mathrm{ea}}(q, t):=\prod_{\substack{s \in \lambda \\ a(s) \text { even }}} b_{\lambda}(s ; q, t)=\prod_{\substack{s \in \lambda \\ a(s) \text { even }}} \frac{1-q^{a(s)} t^{l(s)+1}}{1-q^{a(s)+1} t^{l(s)}} .
$$

Lemma 2.1. Let $\lambda=2\lceil\mu / 2\rceil:=\left(2\left\lceil\mu_{1} / 2\right\rceil, 2\left\lceil\mu_{2} / 2\right\rceil, \ldots\right)$. Then

$$
\psi_{\lambda / \mu}^{\prime}(q, t)=\frac{C_{\lambda / 2}^{-}\left(t ; q^{2}, t\right)}{C_{\lambda / 2}^{-}\left(q ; q^{2}, t\right)} \cdot \frac{1}{b_{\mu}^{\mathrm{ea}}(q, t)} .
$$

Proof. The product in (2.25) is over all squares $s$ in $\mu$ for which $\lambda$ and $\mu$ have the same row but different column length. If $\lambda=2\lceil\mu / 2\rceil$ then $\lambda$ is obtained from $\mu$ by adding a square to each row of odd length. Hence in this case the product is over all squares $s=(i, j)$ such that $\mu_{i}$ and $j$ are both even and such that there exists a $k>i$ such that $\mu_{k}=j-1$. For example, if $\mu=(6,4,3,3,2,1)$ then $\lambda=(6,4,4,4,2,2)$ and the relevant squares contributing to $\prod b_{\lambda}(s ; q, t) / b_{\mu}(s ; q, t)$ are the ones marked with a cross in the two diagrams on the left of the figure below. Because marked squares $s=(i, j)$ occur in even rows and have $j$ even, they must have even arm-length. But we can include all other squares of $\lambda$ and $\mu$ with even arm-length since their contributions to $b_{\lambda}(s ; q, t)$ and $b_{\mu}(s ; q, t)$ trivially cancel, as indicated in the two diagrams on the right:

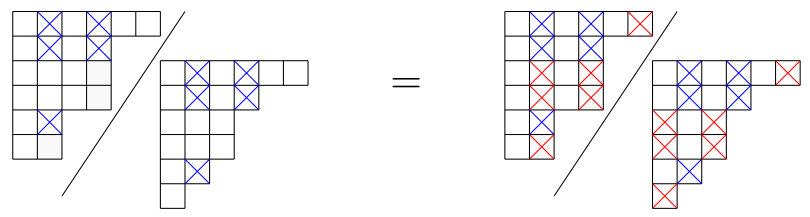

Hence

$$
\begin{aligned}
\psi_{\lambda / \mu}^{\prime}(q, t) & =\prod_{\substack{s \in \lambda \\
a(s) \text { even }}} b_{\lambda}(s ; q, t) \prod_{\substack{s \in \mu \\
a(s) \text { even }}} \frac{1}{b_{\mu}(s ; q, t)} \\
& =\left(\prod_{s \in \lambda / 2} \frac{1-q^{2 a(s)} t^{l(s)+1}}{1-q^{2 a(s)+1} t^{l(s)}}\right) \cdot \frac{1}{b_{\mu}(q, t)}
\end{aligned}
$$

where the second equality uses the fact that $\lambda$ is an even partition. By (2.2) we are done.

Taking $x=\left(x_{1}, \ldots, x_{n}\right)$ and equating terms of degree $|\mu|+n$ in (2.23) we obtain

$$
P_{\mu}\left(x_{1}, \ldots, x_{n} ; q, t\right) x_{1} \cdots x_{n}=\psi_{\left(\mu+1^{n}\right) / \mu}^{\prime}(q, t) P_{\mu+1^{n}}\left(x_{1}, \ldots, x_{n} ; q, t\right) .
$$


By (2.24) the Pieri coefficient on the right is 1 so that

$$
P_{\mu}\left(x_{1}, \ldots, x_{n} ; q, t\right) x_{1} \cdots x_{n}=P_{\mu+1^{n}}\left(x_{1}, \ldots, x_{n} ; q, t\right) .
$$

Closely related to the Pieri formulas is the branching rule [64, page 346]

$$
P_{\lambda}\left(x_{1}, \ldots, x_{n} ; q, t\right)=\sum_{\mu \subset \lambda} x_{n}^{|\lambda / \mu|} \psi_{\lambda / \mu}(q, t) P_{\mu}\left(x_{1}, \ldots, x_{n-1} ; q, t\right),
$$

where [64, page 341]

$$
\psi_{\lambda / \mu}(q, t)=\psi_{\lambda^{\prime} / \mu^{\prime}}^{\prime}(t, q) .
$$

We conclude the section on Macdonald polynomials with the principal specialisation formula [64, page 338]

$$
P_{\lambda}\left(1, t, \ldots, t^{n-1} ; q, t\right)=P_{\lambda}\left(\left[\frac{1-t^{n}}{1-t}\right] ; q, t\right)=t^{n(\lambda)} \frac{\left(t^{n} ; q, t\right)_{\lambda}}{C_{\lambda}^{-}(t ; q, t)} .
$$

\subsection{Koornwinder polynomials.}

2.6.1. Koornwinder polynomials. The Koornwinder polynomials [43] are a generalisation of the Macdonald polynomials to the root system $\mathrm{BC}_{n}$. They depend on six parameters, except for $n=1$ when they correspond to the 5parameter Askey-Wilson polynomials [3].

Throughout this section $x=\left(x_{1}, \ldots, x_{n}\right)$. Then the Koornwinder density is given by

$$
\Delta\left(x ; q, t ; t_{0}, t_{1}, t_{2}, t_{3}\right):=\prod_{i=1}^{n} \frac{\left(x_{i}^{ \pm 2} ; q\right)_{\infty}}{\prod_{r=0}^{3}\left(t_{r} x_{i}^{ \pm} ; q\right)_{\infty}} \prod_{1 \leqslant i<j \leqslant n} \frac{\left(x_{i}^{ \pm} x_{j}^{ \pm} ; q\right)_{\infty}}{\left(t x_{i}^{ \pm} x_{j}^{ \pm} ; q\right)_{\infty}}
$$

where

$$
\begin{aligned}
\left(x_{i}^{ \pm} ; q\right)_{\infty} & :=\left(x_{i}, x_{i}^{-1} ; q\right)_{\infty} \\
\left(x_{i}^{ \pm} x_{j}^{ \pm} ; q\right)_{\infty} & :=\left(x_{i} x_{j}, x_{i} x_{j}^{-1}, x_{i}^{-1} x_{j}, x_{i}^{-1} x_{j}^{-1} ; q\right)_{\infty} .
\end{aligned}
$$

For complex $q, t, t_{0}, \ldots, t_{3}$ such that $|q|,|t|,\left|t_{0}\right|, \ldots,\left|t_{3}\right|<1$ this defines a scalar product on $\mathbb{C}\left[x^{ \pm 1}\right]$ via

$$
\langle f, g\rangle_{q, t ; t_{0}, t_{1}, t_{2}, t_{3}}^{(n)}:=\int_{\mathbb{T}^{n}} f(x) g\left(x^{-1}\right) \Delta\left(x ; q, t ; t_{0}, t_{1}, t_{2}, t_{3}\right) \mathrm{d} T(x),
$$

where

$$
\mathrm{d} T(x):=\frac{1}{2^{n} n !(2 \pi \mathrm{i})^{n}} \frac{\mathrm{d} x_{1}}{x_{1}} \cdots \frac{\mathrm{d} x_{n}}{x_{n}} .
$$

Let $W=\mathfrak{S}_{n} \ltimes(\mathbb{Z} / 2 \mathbb{Z})^{n}$ be the hyperoctahedral group with natural action on $\mathbb{C}\left[x^{ \pm}\right]$. For $\lambda$ a partition of length at most $n$, let $m_{\lambda}^{W}$ be the $W$-invariant monomial symmetric function

$$
m_{\lambda}^{W}(x):=\sum_{\alpha} x^{\alpha}
$$


summed over all $\alpha$ in the $W$-orbit of $\lambda$. In analogy with the Macdonald polynomials, the Koornwinder polynomials $K_{\lambda}=K_{\lambda}\left(x ; q, t ; t_{0}, t_{1}, t_{2}, t_{3}\right)$ are defined as the unique family of polynomials in $\Lambda^{\mathrm{BC}_{n}}:=\mathbb{C}\left[x^{ \pm}\right]^{W}$ such that 43$]$

$$
K_{\lambda}=m_{\lambda}^{W}+\sum_{\mu<\lambda} c_{\lambda \mu} m_{\mu}^{W}
$$

and

$$
\left\langle K_{\lambda}, K_{\mu}\right\rangle_{q, t ; t_{0}, t_{1}, t_{2}, t_{3}}^{(n)}=0 \quad \text { if } \lambda \neq \mu .
$$

From the definition it follows that the $K_{\lambda}$ are symmetric under permutation of the $t_{r}$. The quadratic norm was first evaluated in [23] (selfdual case) and [85] (general case). For our purposes we only need

$$
\langle 1,1\rangle_{q, t ; t_{0}, t_{1}, t_{2}, t_{3}}^{(n)}=\prod_{i=1}^{n} \frac{\left(t, t_{0} t_{1} t_{2} t_{3} t^{n+i-2} ; q\right)_{\infty}}{\left(q, t^{i} ; q\right)_{\infty} \prod_{0 \leqslant r<s \leqslant 3}\left(t_{r} t_{s} t^{i-1} ; q\right)_{\infty}},
$$

known as Gustafson's integral 32.

The $\mathrm{BC}_{n}$ analogue of the Cauchy identity (2.20), is given by [70, Theorem $2.1]$

$$
\begin{aligned}
\sum_{\lambda \subset m^{n}}(-1)^{|\lambda|} & K_{m^{n}-\lambda}\left(x ; q, t ; t_{0}, t_{1}, t_{2}, t_{3}\right) K_{\lambda^{\prime}}\left(y ; t, q ; t_{0}, t_{1}, t_{2}, t_{3}\right) \\
& =\prod_{i=1}^{n} \prod_{j=1}^{m}\left(x_{i}+x_{i}^{-1}-y_{j}-y_{j}^{-1}\right)=\prod_{i=1}^{n} \prod_{j=1}^{m} x_{i}^{-1}\left(1-x_{i} y_{j}^{ \pm}\right),
\end{aligned}
$$

where $y=\left(y_{1}, \ldots, y_{m}\right)$ and $\left(a-b^{ \pm}\right):=(a-b)\left(a-b^{-1}\right)$.

2.6.2. Lifted and virtual Koornwinder polynomials. The lifted Koornwinder polynomials $\tilde{K}_{\lambda}=\tilde{K}_{\lambda}\left(q, t, T ; t_{0}, t_{1}, t_{2}, t_{3}\right)=\tilde{K}_{\lambda}\left(x ; q, t, T ; t_{0}, t_{1}, t_{2}, t_{3}\right)$ are a 7 parameter family of inhomogeneous symmetric functions [77]. They are invariant under permutations of the $t_{r}$ and form a $\mathbb{Q}\left(q, t, T, t_{0}, t_{1}, t_{2}, t_{3}\right)$ basis of $\Lambda$. For example, $\tilde{K}_{0}=1$ and

$$
\tilde{K}_{1}\left(q, t, T ; t_{0}, t_{1}, t_{2}, t_{3}\right)=m_{1}+\frac{1-T}{(1-t)\left(1-t_{0} t_{1} t_{2} t_{3} T^{2} / t^{2}\right)} \sum_{r=0}^{3}\left(\frac{t_{0} t_{1} t_{2} t_{3} T}{t_{r} t}-t_{r}\right) \text {. }
$$

As a function of the $t_{r}$ the lifted Koornwinder polynomial $\tilde{K}_{\lambda}$ has poles at

$$
t_{0} t_{1} t_{2} t_{3}=q^{2-\lambda_{i}-j} t^{i+\lambda_{j}^{\prime}} T^{-2}, \quad(i, j) \in \lambda .
$$


Importantly, according to [77, Theorem 7.1], for generic $q, t, t_{0}, \ldots, t_{3}$ (so as to avoid potential poles)

$$
\begin{aligned}
\tilde{K}_{\lambda}\left(x_{1}^{ \pm}, \ldots, x_{n}^{ \pm} ; q, t, t^{n} ;\right. & \left.t_{0}, t_{1}, t_{2}, t_{3}\right) \\
& = \begin{cases}K_{\lambda}\left(x_{1}, \ldots, x_{n} ; q, t ; t_{0}, t_{1}, t_{2}, t_{3}\right) & \text { if } l(\lambda) \leqslant n \\
0 & \text { otherwise }\end{cases}
\end{aligned}
$$

Let $\hat{\Lambda}$ be the completion of the ring of symmetric functions with respect to the natural grading by degree, i.e., $\hat{\Lambda}$ is the inverse limit of $\Lambda_{n}$ relative to the homomorphism $\rho_{m, n}: \Lambda_{m} \rightarrow \Lambda_{n}(m \geqslant n)$ which sends $m_{\lambda}\left(x_{1}, \ldots, x_{m}\right)$ to $m_{\lambda}\left(x_{1}, \ldots, x_{n}\right)$ for $l(\lambda) \leqslant n$ and to 0 otherwise. Then the virtual Koornwinder polynomials $\hat{K}_{\lambda}=\hat{K}_{\lambda}\left(q, t, Q ; t_{0}, t_{1}, t_{2}, t_{3}\right)=\hat{K}_{\lambda}\left(x ; q, t, Q ; t_{0}, t_{1}, t_{2}, t_{3}\right)$ (which are again symmetric in the $\left.t_{r}\right)$ form a $\mathbb{Q}\left(q, t, Q, t_{0}, t_{1}, t_{2}, t_{3}\right)$ basis of $\hat{\Lambda}$, such that for $x=\left(x_{1}, \ldots, x_{n}\right)$ and $\lambda \subset m^{n}$,

$$
\hat{K}_{\lambda}\left(x ; q, t, q^{m} ; t_{0}, t_{1}, t_{2}, t_{3}\right)=\left(x_{1} \cdots x_{n}\right)^{m} K_{m^{n}-\lambda}\left(x ; q, t ; t_{0}, t_{1}, t_{2}, t_{3}\right) .
$$

When $Q=0$ the virtual Koornwinder polynomials can be expressed in terms of Macdonald polynomials as [77, Corollary 7.21]

$$
\hat{K}_{\lambda}\left(x ; q, t, 0 ; t_{0}, t_{1}, t_{2}, t_{3}\right)=P_{\lambda}(x ; q, t) \prod_{i=1}^{n} \frac{\prod_{r=0}^{3}\left(t_{r} x_{i} ; q\right)_{\infty}}{\left(x_{i}^{2} ; q\right)_{\infty}} \prod_{1 \leqslant i<j \leqslant n} \frac{\left(t x_{i} x_{j} ; q\right)_{\infty}}{\left(x_{i} x_{j} ; q\right)_{\infty}}
$$

from which it follows that

$$
\begin{aligned}
\lim _{m \rightarrow \infty}\left(x_{1} \cdots x_{n}\right)^{m} & K_{m^{n}-\lambda}\left(x ; q, t ; t_{0}, t_{1}, t_{2}, t_{3}\right) \\
& =P_{\lambda}(x ; q, t) \prod_{i=1}^{n} \frac{\prod_{r=0}^{3}\left(t_{r} x_{i} ; q\right)_{\infty}}{\left(x_{i}^{2} ; q\right)_{\infty}} \prod_{1 \leqslant i<j \leqslant n} \frac{\left(t x_{i} x_{j} ; q\right)_{\infty}}{\left(x_{i} x_{j} ; q\right)_{\infty}} .
\end{aligned}
$$

The lifted and virtual Koornwinder polynomials admit a lift of the Cauchy identity (2.34) to $\hat{\Lambda}_{x} \otimes \Lambda_{y}$ as follows [77, Theorem 7.14]:

$$
\begin{aligned}
\sum_{\lambda}(-1)^{|\lambda|} \hat{K}_{\lambda}\left(x ; t, q, T ; t_{0}, t_{1}, t_{2}, t_{3}\right) \tilde{K}_{\lambda^{\prime}}\left(y ; q, t, T ; t_{0}, t_{1}, t_{2}, t_{3}\right) & \\
& =\prod_{i, j \geqslant 1}\left(1-x_{i} y_{j}\right) .
\end{aligned}
$$

This may be used to derive the following symmetry relation for virtual Koornwinder polynomials. 
Proposition 2.2. The virtual Koornwinder polynomials satisfy

$$
\begin{aligned}
\hat{K}_{\lambda}(x ; q, t, Q ; & \left.t_{0}, t_{1}, t_{2}, t_{3}\right) \\
& =\hat{K}_{\lambda}\left(x ; q, t, Q t_{0} t_{1} / q ; q / t_{0}, q / t_{1}, t_{2}, t_{3}\right) \prod_{i \geqslant 1} \frac{\left(t_{0} x_{i}, t_{1} x_{i} ; q\right)_{\infty}}{\left(q x_{i} / t_{0}, q x_{i} / t_{1} ; q\right)_{\infty}} .
\end{aligned}
$$

Proof. We begin with (2.39) and identify the product on the right as $\sigma_{1}[-x y]$. Carrying out the plethystic substitution

$$
y \mapsto y+\frac{t_{0}-t / t_{0}}{1-t}+\frac{t_{1}-t / t_{1}}{1-t}
$$

and applying the symmetry [77, Equation (7.2)]

$$
\begin{aligned}
\tilde{K}_{\lambda}\left(\left[y+\frac{t_{0}-t / t_{0}}{1-t}+\frac{t_{1}-t / t_{1}}{1-t}\right]\right. & \left.; q, t, T ; t_{0}, t_{1}, t_{2}, t_{3}\right) \\
& =\tilde{K}_{\lambda}\left(y ; q, t, T t_{0} t_{1} / t ; t / t_{0}, t / t_{1}, t_{2}, t_{3}\right)
\end{aligned}
$$

we obtain

$$
\begin{aligned}
\sum_{\lambda}(-1)^{|\lambda|} \hat{K}_{\lambda}\left(x ; t, q, T ; t_{0}, t_{1}, t_{2}, t_{3}\right) \tilde{K}_{\lambda^{\prime}}\left(y ; q, t, T t_{0} t_{1} / t ; t / t_{0}, t / t_{1}, t_{2}, t_{3}\right) \\
=\prod_{i \geqslant 1} \frac{\left(t_{0} x_{i}, t_{1} x_{i} ; t\right)_{\infty}}{\left(t x_{i} / t_{0}, t x_{i} / t_{1} ; t\right)_{\infty}} \prod_{i, j \geqslant 1}\left(1-x_{i} y_{j}\right) .
\end{aligned}
$$

Here we have also used (2.14c) to transform the right-hand side. After replacing $\left(t_{0}, t_{1}, T\right) \mapsto\left(t / t_{0}, t / t_{1}, T t_{0} t_{1} / t\right)$ this is equivalent to

$$
\begin{aligned}
\sum_{\lambda}(-1)^{|\lambda|} \hat{K}_{\lambda}\left(x ; t, q, T t_{0} t_{1} / t ; t / t_{0}, t / t_{1}, t_{2}, t_{3}\right) \tilde{K}_{\lambda^{\prime}}\left(y ; q, t, T ; t_{0}, t_{1}, t_{2}, t_{3}\right) \\
=\prod_{i \geqslant 1} \frac{\left(t x_{i} / t_{0}, t x_{i} / t_{1} ; t\right)_{\infty}}{\left(t_{0} x_{i}, t_{1} x_{i} ; t\right)_{\infty}} \prod_{i, j \geqslant 1}\left(1-x_{i} y_{j}\right) .
\end{aligned}
$$

Expanding the double product on the right by the Cauchy identity (2.39) and extracting coefficients of $\tilde{K}_{\lambda^{\prime}}\left(y ; q, t, T ; t_{0}, t_{1}, t_{2}, t_{3}\right)$ yields

$$
\begin{aligned}
\hat{K}_{\lambda}\left(x ; t, q, T t_{0} t_{1} / t ; t / t_{0}, t /\right. & \left.t_{1}, t_{2}, t_{3}\right) \\
& =\hat{K}_{\lambda}\left(x ; t, q, T ; t_{0}, t_{1}, t_{2}, t_{3}\right) \prod_{i \geqslant 1} \frac{\left(t x_{i} / t_{0}, t x_{i} / t_{1} ; t\right)_{\infty}}{\left(t_{0} x_{i}, t_{1} x_{i} ; t\right)_{\infty}} .
\end{aligned}
$$

By the change $(t, q, T) \mapsto(q, t, Q)$ we are done.

\subsection{Macdonald-Koornwinder polynomials.}


2.7.1. Macdonald polynomials on root systems. Below we closely follow the exposition in [65].

Let $E$ be a Euclidean space with positive-definite symmetric bilinear form $\langle\cdot, \cdot\rangle$ and $R$ a root system spanning $E$. The rank of $R$ is the dimension of $E$. All root systems will be assumed to be irreducible, but not necessarily reduced. The root system dual to $R$, denoted $R^{\vee}$, is given by

$$
R^{\vee}=\left\{\alpha^{\vee}: \alpha \in R\right\},
$$

where $v^{\vee}:=2 v /\langle v, v\rangle=2 v /\|v\|^{2}$ for $v \in E$.

A pair of root systems $(R, S)$ in $E$ is called admissible if $R$ and $S$ share the same Weyl group $W$ and $S$ is reduced. Given such an admissible pair and $\alpha \in R$, there exists a unique $u_{\alpha}>0$ such that $u_{\alpha}^{-1} \alpha \in S$. Moreover, the map $\alpha \mapsto u_{\alpha}^{-1} \alpha$ from $R$ to $S$ is surjective (injective if $R$ is reduced) and commutes with the action of $W$. Hence $u_{\alpha}$ is fixed along Weyl orbits, and $u_{2 \alpha}=2 u_{\alpha}$ if $\alpha, 2 \alpha$ are both in $R$. In the following we only need admissible pairs $(R, S)$ up to similarity, allowing for the scaling of the $u_{\alpha}$ by a positive constant. The classification of admissible pairs then breaks up into three cases.

(1) $R$ is simply laced and $S=R$ (and hence $u_{\alpha}=1$ ).

(2) $R$ is reduced but not simply laced and $S=R^{\vee}$. Unlike Macdonald, who normalises short roots of $R$ to have length $\sqrt{2}$, we take the length of the short root to be 1 when $R=\mathrm{B}_{n}$ and $\sqrt{2}$ in all other cases. Then the $u_{\alpha}$ are 1 (long roots) or $1 / 2$ (short root) when $R=\mathrm{B}_{n}, 1$ (short roots) or 2 (long roots) when $R=\mathrm{C}_{n}, 1$ (short) or 2 (long) when $R=\mathrm{F}_{4}$, and 1 (short) or 3 (long) when $R=\mathrm{G}_{2}$.

(3) $R$ is the non-reduced root system $\mathrm{BC}_{n}$ and $S$ is one of $\mathrm{B}_{n}, \mathrm{C}_{n}$. In the $\mathrm{B}_{n}$ case we normalise $S=\mathrm{B}_{n} \subset \mathrm{BC}_{n}=R$ and in the $\mathrm{C}_{n}$ case $S=\mathrm{C}_{n} \subset \frac{1}{2} R=\frac{1}{2} \mathrm{BC}_{n}$. Then $u_{\alpha} \in\{1,2\}$ for all $\alpha$.

For $(R, S)$ an admissible pair of root systems of rank $r$ we fix a basis of simple roots $\Delta=\left\{\alpha_{1}, \ldots, \alpha_{r}\right\}$ of $R$, and write $\alpha>0$ if $\alpha \in R$ is a positive root with respect to this basis. The fundamental weights $\Lambda_{1}, \ldots, \Lambda_{r}$ of $R$ are given by $\left\langle\alpha_{i}^{\vee}, \Lambda_{j}\right\rangle=\delta_{i j}$. As usual we denote the root and weight lattices of $R$ by $Q$ and $P$ respectively, and write $Q^{+}=\sum_{i=1}^{r} \mathbb{Z}_{\geqslant 0} \alpha_{i}$ and $P_{+}=\sum_{i=1}^{r} \mathbb{Z}_{\geqslant 0} \Lambda_{i}$ for the set of dominant (integral) weights.

Let $A$ be the group algebra over $\mathbb{R}$ of $P$ with elements $\mathrm{e}^{\lambda}$ and $A^{W}$ the algebra of $W$-invariant elements of $A$. A basis of $A^{W}$ is given by the monomial symmetric functions

$$
m_{\lambda}^{W}=\sum \mathrm{e}^{\mu}, \quad \lambda \in P_{+},
$$

with sum over the $W$-orbit of $\lambda$.

The $(R, S)$ Macdonald polynomials defined below depend on the variables $q$ and $t_{\alpha}, \alpha \in R$, such that $t_{\alpha}$ is constant along Weyl orbits. Hence there is only one $t_{\alpha}$ in case (1), two in case (2), and three in case (3). In each case we 
write this set of $t_{\alpha}$ 's by $\underline{t}$. The generalised Macdonald density (compare with (2.17)) is then

$$
\Delta(q, \underline{t}):=\prod_{\alpha \in R} \frac{\left(t_{2 \alpha}^{1 / 2} \mathrm{e}^{\alpha} ; q^{u_{\alpha}}\right)_{\infty}}{\left(t_{\alpha} t_{2 \alpha}^{1 / 2} \mathrm{e}^{\alpha} ; q^{u_{\alpha}}\right)_{\infty}},
$$

where $t_{2 \alpha}:=1$ if $2 \alpha \notin R$. Assuming, $|q|,\left|t_{\alpha}\right|<1$ this defines the following scalar product on $A$ :

$$
\langle f, g\rangle_{q, \underline{t}}:=\frac{1}{|W|} \int_{T} f \bar{g} \Delta(q, \underline{t}),
$$

where integration is with respect to Haar measure on the torus $T=E / Q^{\vee}$ and, for $f=\sum_{\lambda \in P} f_{\lambda} \mathrm{e}^{\lambda}, \bar{f}:=\sum_{\lambda \in P} f_{\lambda} \mathrm{e}^{-\lambda}$. The $(R, S)$ Macdonald polynomials $P_{\lambda}(q, \underline{t}), \lambda \in P_{+}$then arise as the unique family of $A^{W}$-symmetric functions

$$
P_{\lambda}(q, \underline{t})=m_{\lambda}^{W}+\sum_{\mu<\lambda} u_{\lambda \mu}(q, \underline{t}) m_{\mu}^{W}
$$

such that

$$
\left\langle P_{\lambda}, P_{\mu}\right\rangle_{q, \underline{t}}=0 \quad \text { if } \quad \lambda \neq \mu .
$$

The sum in (2.42) is with respect to the dominance (partial) order on $P_{+}$ defined by $\lambda \geqslant \mu$ if $\lambda-\mu \in Q^{+}$.

Below we are interested in the generalised Macdonald polynomials for $(R, S)$ one of $\left(\mathrm{B}_{n}, \mathrm{~B}_{n}\right),\left(\mathrm{B}_{n}, \mathrm{C}_{n}\right),\left(\mathrm{C}_{n}, \mathrm{~B}_{n}\right)$ and $\left(\mathrm{D}_{n}, \mathrm{D}_{n}\right)$. Moreover, in the HallLittlewood limit, $q \rightarrow 0$, (in which case the $S$ dependence drops out) we also need $R=\mathrm{BC}_{n}$. In the following we assume the standard realisation of $\mathrm{B}_{n}, \mathrm{C}_{n}$ and $\mathrm{D}_{n}$ in $\mathbb{R}^{n}$ (consistent with our normalisation in (2)):

$$
\Delta=\left\{\alpha_{1}, \ldots, \alpha_{n}\right\}=\left\{\epsilon_{1}-\epsilon_{2}, \ldots, \epsilon_{n-1}-\epsilon_{n}, \epsilon_{n}\right\}, \quad R=\mathrm{B}_{n}=\mathrm{C}_{n}^{\vee},
$$

$$
\Delta=\left\{\alpha_{1}, \ldots, \alpha_{n}\right\}=\left\{\epsilon_{1}-\epsilon_{2}, \ldots, \epsilon_{n-1}-\epsilon_{n}, \epsilon_{n-1}+\epsilon_{n}\right\}, \quad R=\mathrm{D}_{n},
$$

and parametrise the set of dominant weights $P_{+}$as

$$
\begin{array}{ll}
\left(\lambda_{1}-\lambda_{2}\right) \Lambda_{1}+\cdots+\left(\lambda_{n-1}-\lambda_{n}\right) \Lambda_{n-1}+2 \lambda_{n} \Lambda_{n}, & R=\mathrm{B}_{n} \\
\left(\lambda_{1}-\lambda_{2}\right) \Lambda_{1}+\cdots+\left(\lambda_{n-1}-\lambda_{n}\right) \Lambda_{n-1}+\lambda_{n} \Lambda_{n}, & R=\mathrm{C}_{n} \\
\left(\lambda_{1}-\lambda_{2}\right) \Lambda_{1}+\cdots+\left(\lambda_{n-1}-\lambda_{n}\right) \Lambda_{n-1}+\left(\lambda_{n-1}+\lambda_{n}\right) \Lambda_{n}, & R=\mathrm{D}_{n}
\end{array}
$$

where $\lambda$ is a partition in the case of $\mathrm{C}_{n}$ and a partition or half-partition in the case of $\mathrm{B}_{n}, \mathrm{D}_{n}$ with the exception that for $\mathrm{D}_{n}$ the part $\lambda_{n}$ can be negative: $-\lambda_{n-1} \leqslant \lambda_{n} \leqslant \lambda_{n-1}$ 2 Finally writing $x_{i}=\mathrm{e}^{-\epsilon_{i}}($ for $1 \leqslant i \leqslant n$ ), we will denote

\footnotetext{
${ }^{2}$ The map $\lambda_{n} \mapsto-\lambda_{n}$ corresponds to the Dynkin diagram automorphism interchanging $\Lambda_{n-1}$ and $\Lambda_{n}$.
} 
the four families of interest by

$$
P_{\lambda}^{\left(\mathrm{B}_{n}, \mathrm{~B}_{n}\right)}\left(x ; q, t, t_{2}\right), P_{\lambda}^{\left(\mathrm{B}_{n}, \mathrm{C}_{n}\right)}\left(x ; q, t, t_{2}\right), P_{\lambda}^{\left(\mathrm{C}_{n}, \mathrm{~B}_{n}\right)}\left(x ; q, t, t_{2}\right), P_{\lambda}^{\left(\mathrm{D}_{n}, \mathrm{D}_{n}\right)}(x ; q, t),
$$

where $x=\left(x_{1}, \ldots, x_{n}\right)$ and $\left(t, t_{2}\right):=\left(t_{\alpha_{1}}, t_{\alpha_{n}}\right)$ in the first three cases and $t=t_{\alpha_{1}}$ for $\mathrm{D}_{n}$.

There are several relations between the polynomials of interest. For example, if $\bar{\lambda}:=\left(\lambda_{1}, \ldots, \lambda_{n-1},-\lambda_{n}\right)$ then [22, Equation (5.60)]

$$
P_{\lambda}^{\left(\mathrm{D}_{n}, \mathrm{D}_{n}\right)}(x ; q, t)=P_{\lambda}^{\left(\mathrm{B}_{n}, \mathrm{~B}_{n}\right)}(x ; q, t, 1)
$$

if $l(\lambda)<n$ and

$$
\begin{aligned}
& P_{\lambda}^{\left(\mathrm{D}_{n}, \mathrm{D}_{n}\right)}(x ; q, t)+P_{\bar{\lambda}}^{\left(\mathrm{D}_{n}, \mathrm{D}_{n}\right)}(x ; q, t)=P_{\lambda}^{\left(\mathrm{B}_{n}, \mathrm{~B}_{n}\right)}(x ; q, t, 1) \\
& P_{\lambda}^{\left(\mathrm{D}_{n}, \mathrm{D}_{n}\right)}(x ; q, t)-P_{\bar{\lambda}}^{\left(\mathrm{D}_{n}, \mathrm{D}_{n}\right)}(x ; q, t)=P_{\lambda-\left(\frac{1}{2}\right)^{n}}^{\left(\mathrm{B}_{n}, \mathrm{C}_{n}\right)}\left(x ; q, t, q^{1 / 2}\right) \\
& \times \prod_{i=1}^{n}\left(x_{i}^{-1 / 2}-x_{i}^{1 / 2}\right)
\end{aligned}
$$

if $\lambda$ is a partition or half-partition of length $n$. Whence

$$
P_{\bar{\lambda}}^{\left(\mathrm{D}_{n}, \mathrm{D}_{n}\right)}(x ; q, t)=P_{\lambda}^{\left(\mathrm{D}_{n}, \mathrm{D}_{n}\right)}(\bar{x} ; q, t), \quad \bar{x}:=\left(x_{1}, \ldots, x_{n-1}, x_{n}^{-1}\right) .
$$

Similarly, comparing the Koornwinder density (2.31) with (2.41) it follows that

$$
P_{\lambda}^{\left(\mathrm{C}_{n}, \mathrm{~B}_{n}\right)}\left(x ; q, t, t_{2}\right)=K_{\lambda}\left(x ; q, t ; \pm q^{1 / 2}, \pm t_{2}^{1 / 2}\right)
$$

(see also [22]). Although the $\left(\mathrm{B}_{n}, S\right)$ Macdonald polynomials are indexed by partitions or half-partitions they too may be related to Koornwinder polynomials [22]. Before describing this relation, we briefly discuss another family of polynomials incorporating both $\mathrm{B}_{n}$ families.

2.7.2. The Macdonald-Koornwinder polynomials $K_{\lambda}\left(x ; q, t, t_{2}, t_{3}\right)$. Our description of Macdonald polynomials attached to root systems is by no means the most general and modern setup, see e.g., [16, 66, 89]. Beyond Macdonald's original approach we have already covered the Koornwinder polynomials. Here we discuss one further family of $\mathrm{B}_{n}$-like polynomials which we shall denote by $K_{\lambda}\left(x ; q, t ; t_{2}, t_{3}\right)$. In the notation of [89] they correspond to the MacdonaldKoornwinder polynomials $P_{\lambda}^{+}$of [89, Definition 3.21] with initial data given by the quintuple $D=\left(\mathrm{B}_{n}, \Delta, t, P, Q\right)$. Here $\Delta$ is the basis of simple roots of $\mathrm{B}_{n}$ given in (2.43a), $P$ and $Q$ again denote the weight and root lattices of $\mathrm{B}_{n}$, and $t$ stands for 'twisted'.

The polynomials $K_{\lambda}\left(x ; q, t ; t_{2}, t_{3}\right)$, where $x=\left(x_{1}, \ldots, x_{n}\right)$ and $\lambda=\left(\lambda_{1}, \ldots, \lambda_{n}\right)$ is a partition or half-partition, are $\mathrm{B}_{n}$-symmetric functions (in the sense of (2.42) ) such that

$$
\left\langle K_{\lambda}\left(x ; q, t ; t_{2}, t_{3}\right), K_{\mu}\left(x ; q, t ; t_{2}, t_{3}\right)\right\rangle_{q, t ; t_{2}, t_{3}}^{(n)}=0 .
$$


Here, for $f, g \in \Lambda^{\mathrm{BC}_{n}}$,

$$
\langle f, g\rangle_{q, t ; t_{2}, t_{3}}^{(n)}:=\int_{\mathbb{T}^{n}} f(x) g(x) \Delta\left(x ; q, t ; t_{2}, t_{3}\right) \mathrm{d} T(x)
$$

with

$$
\Delta\left(x ; q, t ; t_{2}, t_{3}\right):=\prod_{i=1}^{n} \frac{\left(x_{i}^{ \pm} ; q^{1 / 2}\right)_{\infty}}{\left(t_{2} x_{i}^{ \pm}, t_{3} x_{i}^{ \pm} ; q\right)_{\infty}} \prod_{1 \leqslant i<j \leqslant n} \frac{\left(x_{i}^{ \pm} x_{j}^{ \pm} ; q\right)_{\infty}}{\left(t x_{i}^{ \pm} x_{j}^{ \pm} ; q\right)_{\infty}}
$$

In the notation of the previous section this corresponds to $(R, S)=\left(\mathrm{B}_{n}, \mathrm{~B}_{n}\right)$ and

$$
\Delta(q, \underline{t})=\prod_{\alpha \text { short }} \frac{\left(\mathrm{e}^{\alpha}, q^{1 / 2} \mathrm{e}^{\alpha} ; q\right)_{\infty}}{\left(t_{\alpha} \mathrm{e}^{\alpha}, \bar{t}_{\alpha} \mathrm{e}^{\alpha} ; q\right)_{\infty}} \prod_{\alpha \text { long }} \frac{\left(\mathrm{e}^{\alpha} ; q\right)_{\infty}}{\left(t_{\alpha} \mathrm{e}^{\alpha} ; q\right)_{\infty}}
$$

where $\underline{t}=\left(t_{\alpha_{1}}, t_{\alpha_{n}}, \bar{t}_{\alpha_{n}}\right)=\left(t, t_{2}, t_{3}\right)$. It thus follows that

$$
\begin{aligned}
& P_{\lambda}^{\left(\mathrm{B}_{n}, \mathrm{~B}_{n}\right)}\left(x ; q, t, t_{2}\right)=K_{\lambda}\left(x ; q, t ; t_{2}, q^{1 / 2}\right) \\
& P_{\lambda}^{\left(\mathrm{B}_{n}, \mathrm{C}_{n}\right)}\left(x ; q, t, t_{2}\right)=K_{\lambda}\left(x ; q, t ; t_{2}, t_{2} q^{1 / 2}\right) .
\end{aligned}
$$

The next lemma shows that the $K_{\lambda}\left(x ; q, t ; t_{2}, t_{3}\right)$ can be expressed in terms of Koornwinder polynomials, allowing us to prove results for the former using the latter.

Lemma 2.3. For $\lambda$ a partition or half-partition

$$
\begin{aligned}
& K_{\lambda}\left(x ; q, t ; t_{2}, t_{3}\right) \\
& = \begin{cases}K_{\lambda}\left(x ; q, t ;-1,-q^{1 / 2}, t_{2}, t_{3}\right) & \lambda \text { a partition } \\
K_{\lambda-\left(\frac{1}{2}\right)^{n}}\left(x ; q, t ;-q,-q^{1 / 2}, t_{2}, t_{3}\right) \prod_{i=1}^{n}\left(x_{i}^{1 / 2}+x_{i}^{-1 / 2}\right) & \text { otherwise. }\end{cases}
\end{aligned}
$$

For $t_{3}=q^{1 / 2}$ or $t_{3}=t_{2} q^{1 / 2}$ this is equivalent to [22, Equations (5.50) \& (5.51)].

Proof. The triangularity with respect to the $\mathrm{B}_{n}$-symmetric monomial symmetric functions is clear and in the following we show that $K_{\lambda}\left(x ; q, t ; t_{2}, t_{3}\right)$ as given by the lemma satisfies (2.48). Viewing the integral on the right of (2.49) as a constant term evaluation, it follows that (2.48) holds when $\lambda$ is a partition and $\mu$ a half-partition. By (2.32) with $\left\{t_{0}, t_{1}\right\}=\left\{-1,-q^{1 / 2}\right\}$ it is also clear that it 
holds when $\lambda$ and $\mu$ are both partitions. In the case of two half-partitions

$$
\begin{aligned}
& \left\langle K_{\lambda}\left(x ; q, t ; t_{2}, t_{3}\right), K_{\mu}\left(x ; q, t ; t_{2}, t_{3}\right)\right\rangle_{q, t ; t_{2}, t_{3}}^{(n)} \\
& =\int_{\mathbb{T}^{n}} K_{\nu}\left(x ; q, t ;-q,-q^{1 / 2}, t_{2}, t_{3}\right) K_{\omega}\left(x ; q, t ;-q,-q^{1 / 2}, t_{2}, t_{3}\right) \\
& \quad \times \prod_{i=1}^{n}\left(x_{i}^{1 / 2}+x_{i}^{-1 / 2}\right)^{2} \frac{\left(x_{i}^{ \pm} ; q^{1 / 2}\right)_{\infty}}{\left(t_{2} x_{i}^{ \pm}, t_{3} x_{i}^{ \pm} ; q\right)_{\infty}} \prod_{1 \leqslant i<j \leqslant n} \frac{\left(x_{i}^{ \pm} x_{j}^{ \pm} ; q\right)_{\infty}}{\left(t x_{i}^{ \pm} x_{j}^{ \pm} ; q\right)_{\infty}} \mathrm{d} T(x),
\end{aligned}
$$

where $\nu:=\lambda-\left(\frac{1}{2}\right)^{n}$ and $\omega:=\mu-\left(\frac{1}{2}\right)^{n}$. Recall that $\left(1-u^{ \pm}\right):=(1-u)\left(1-u^{-1}\right)$. Since

$$
\left(u^{1 / 2}+u^{-1 / 2}\right)^{2}=\left(1+u^{ \pm}\right)=\frac{\left(-u^{ \pm} ; q^{1 / 2}\right)_{\infty}}{\left(-q u^{ \pm},-q^{1 / 2} u^{ \pm} ; q\right)_{\infty}}
$$

and

$$
\left(-u^{ \pm}, u^{ \pm} ; q^{1 / 2}\right)_{\infty}=\left(u^{ \pm 2} ; q\right)_{\infty}
$$

the second line of the integrand is precisely the Koornwinder density

$$
\Delta\left(x ; q, t ;-q,-q^{1 / 2}, t_{2}, t_{3}\right) .
$$

Hence

$$
\begin{aligned}
& \left\langle K_{\lambda}\left(x ; q, t ; t_{2}, t_{3}\right), K_{\mu}\left(x ; q, t ; t_{2}, t_{3}\right)\right\rangle_{q, t ; t_{2}, t_{3}}^{(n)} \\
& \quad\left\langle K_{\nu}\left(x ; q, t ;-q,-q^{1 / 2}, t_{2}, t_{3}\right), K_{\omega}\left(x ; q, t ;-q,-q^{1 / 2}, t_{2}, t_{3}\right)\right\rangle_{q, t ;-q,-q^{1 / 2}, t_{2}, t_{3}}^{(n)}
\end{aligned}
$$

By (2.32) this vanishes unless $\nu=\omega$, i.e., unless $\lambda=\mu$.

That $K_{\lambda-\left(\frac{1}{2}\right)^{n}}\left(x ; q, t ;-q,-q^{1 / 2}, t_{2}, t_{3}\right) \prod_{i}\left(x_{i}^{1 / 2}+x_{i}^{-1 / 2}\right)$ is the natural extension of $K_{\lambda}\left(x ; q, t ;-1,-q^{1 / 2}, t_{2}, t_{3}\right)$ to half-partitions $\lambda$ may also be understood from the point of view of virtual Koornwinder polynomials. To see this take

$$
\left(x ; Q, t_{0}, t_{1}\right)=\left(x_{1}, \ldots, x_{n} ; q^{m},-1,-q^{1 / 2}\right)
$$

in Proposition (2.2). Then

$$
\hat{K}_{\lambda}\left(x ; q, t, q^{m} ;-1,-q^{1 / 2}, t_{2}, t_{3}\right)=\hat{K}_{\lambda}\left(x ; q, t, q^{m-1 / 2} ;-q,-q^{1 / 2}, t_{2}, t_{3}\right) \prod_{i=1}^{n}\left(1+x_{i}\right) \text {. }
$$

Since the left-hand side of (2.37) is well-defined for $\lambda$ a partition and $m$ a half-integer, we can use that equation to eliminate the virtual Koornwinder polynomials in the above formula. Also replacing $m^{n}-\lambda$ by $\lambda$ this results in

$$
K_{\lambda}\left(x ; q, t ;-1,-q^{1 / 2}, t_{2}, t_{3}\right)=K_{\lambda-\left(\frac{1}{2}\right)^{n}}\left(x ; q, t ;-q,-q^{1 / 2}, t_{2}, t_{3}\right) \prod_{i=1}^{n}\left(x_{i}^{1 / 2}+x_{i}^{-1 / 2}\right) .
$$


2.7.3. Hall-Littlewood polynomials. The ordinary (or $\mathrm{A}_{n-1}$ ) Hall-Littlewood polynomials are defined as

$$
P_{\lambda}\left(x_{1}, \ldots, x_{n} ; t\right):=\frac{1}{v_{\lambda}(t)} \sum_{w \in \mathfrak{S}_{n}} w\left(x_{1}^{\lambda_{1}} \cdots x_{n}^{\lambda_{n}} \prod_{1 \leqslant i<j \leqslant n} \frac{x_{i}-t x_{j}}{x_{i}-x_{j}}\right)
$$

where $\lambda$ is a partition of length at most $n, v_{\lambda}(t):=\prod_{i \geqslant 0}(t ; t)_{m_{i}(\lambda)} /(1-t)^{m_{i}(\lambda)}$ and $m_{0}(\lambda):=n-l(\lambda)$. They correspond to the Macdonald polynomials for $q=0$, i.e., $P_{\lambda}(x ; t)=P_{\lambda}(x ; 0, t)$, and for $t=0$ reduce to the Schur functions.

Taking $q=0$ in the $(R, S)$ Macdonald polynomials of Section 2.7.1 yields the more general Hall-Littlewood polynomials of type $R$. (The root system $S$ no longer plays a role when $q=0$.) More simply, however, the Hall-Littlewood polynomials of type $R$ can be explicitly computed from

$$
P_{\lambda}(t)=\frac{1}{W_{\lambda}(t)} \sum_{w \in W} w\left(\mathrm{e}^{\lambda} \prod_{\alpha>0} \frac{1-t_{\alpha} t_{2 \alpha}^{1 / 2} \mathrm{e}^{-\alpha}}{1-t_{2 \alpha}^{1 / 2} \mathrm{e}^{-\alpha}}\right), \quad \lambda \in P_{+} .
$$

The normalising factor $W_{\lambda}(t)$ is the Poincaré polynomial of the stabilizer of $\lambda$ in $W$.

In order to show that our bounded Littlewood identities for Macdonald polynomials reduce to known results in the $q=0$ limit (or in the statement of some new results that do not have a $q$-analogue) we need to consider (2.52) for $R$ one of $\mathrm{BC}_{n}, \mathrm{~B}_{n}, \mathrm{C}_{n}, \mathrm{D}_{n}$. As in Section 2.7.1, we express these four families using variables $x_{1}, \ldots, x_{n}$ and partitions or half-partitions $\lambda$, rather than roots and dominant weights, and write $P_{\lambda}^{(R)}\left(x_{1}, \ldots, x_{n} ; q, t, t_{2}, \ldots, t_{r}\right)$, with $r=3$ in the case of $\mathrm{BC}_{n}, r=2$ for $\mathrm{B}_{n}$ and $\mathrm{C}_{n}$ and $r=0$ for $\mathrm{D}_{n}$. In each case we again assume (2.43) and (2.44) and identify $x_{i}=\exp \left(-\epsilon_{i}\right)$. As basis for $\mathrm{BC}_{n}$ we take the $\mathrm{B}_{n}$ basis (2.43a) (as opposed to a $\mathrm{C}_{n}$ basis) and identify $\left(t_{\alpha_{1}}, t_{\alpha_{n}}, t_{2 \alpha_{n}}^{1 / 2}\right)=\left(t,-t_{2} / t_{3},-t_{3}\right)$. Then (2.52) takes the equivalent form

$$
\begin{aligned}
& P_{\lambda}^{\left(\mathrm{BC}_{n}\right)}\left(x ; t, t_{2}, t_{3}\right)=\frac{1}{\left(t_{2} t_{3} ; t\right)_{n-l(\lambda)} v_{\lambda}(t)} \\
& \times \sum_{w \in W} w\left(x^{-\lambda} \prod_{i=1}^{n} \frac{\left(1-t_{2} x_{i}\right)\left(1-t_{3} x_{i}\right)}{1-x_{i}^{2}} \prod_{1 \leqslant i<j \leqslant n} \frac{\left(t x_{i}-x_{j}\right)\left(1-t x_{i} x_{j}\right)}{\left(x_{i}-x_{j}\right)\left(1-x_{i} x_{j}\right)}\right),
\end{aligned}
$$

with $W$ the hyperoctahedral group and $\lambda$ a partition of length at most $n$. Alternatively (see e.g., [93]),

$$
P_{\lambda}^{\left(\mathrm{BC}_{n}\right)}\left(x ; t, t_{2}, t_{3}\right)=K_{\lambda}\left(x ; 0, t ; 0,0, t_{2}, t_{3}\right) .
$$


For $t=0$ this admits the determinantal form

$$
\begin{aligned}
& P_{\lambda}^{\left(\mathrm{BC}_{n}\right)}\left(x ; 0, t_{2}, t_{3}\right)=\frac{1}{\Delta_{\mathrm{C}}(x)} \\
\times & \operatorname{det}_{1 \leqslant i, j \leqslant n}\left(x_{i}^{-\lambda_{j}+j-1}\left(1-t_{2} x_{i}\right)\left(1-t_{3} x_{i}\right)-x_{i}^{\lambda_{j}+2 n-j-1}\left(x_{i}-t_{2}\right)\left(x_{i}-t_{3}\right)\right),
\end{aligned}
$$

where

$$
\Delta_{\mathrm{C}}(x):=\prod_{i=1}^{n}\left(1-x_{i}^{2}\right) \prod_{1 \leqslant i<j \leqslant n}\left(x_{i}-x_{j}\right)\left(x_{i} x_{j}-1\right)=\operatorname{det}_{1 \leqslant i, j \leqslant n}\left(x_{i}^{j-1}-x_{i}^{2 n-j+1}\right)
$$

is the $\mathrm{C}_{n}$ Vandermonde product.

Lemma 2.4. Let

$$
\Phi\left(x ; t, t_{2}, t_{3}\right):=\prod_{i=1}^{n} \frac{\left(1-t_{2} x_{i}\right)\left(1-t_{3} x_{i}\right)}{1-x_{i}^{2}} \prod_{1 \leqslant i<j \leqslant n} \frac{1-t x_{i} x_{j}}{1-x_{i} x_{j}}
$$

and $m$ a positive integer. Then

$$
P_{m^{n}}^{\left(\mathrm{BC}_{n}\right)}\left(x ; t, t_{2}, t_{3}\right)=\sum_{\varepsilon \in\{ \pm 1\}^{n}} \Phi\left(x^{\varepsilon} ; t, t_{2}, t_{3}\right) \prod_{i=1}^{n} x_{i}^{-\varepsilon_{i} m} .
$$

Proof. By (2.51) and (2.57),

$$
\begin{aligned}
& \sum_{w \in \mathfrak{S}_{n}} w\left(x^{-\lambda} \prod_{i=1}^{n} \frac{\left(1-t_{2} x_{i}\right)\left(1-t_{3} x_{i}\right)}{1-x_{i}^{2}} \prod_{1 \leqslant i<j \leqslant n} \frac{\left(t x_{i}-x_{j}\right)\left(1-t x_{i} x_{j}\right)}{\left(x_{i}-x_{j}\right)\left(1-x_{i} x_{j}\right)}\right) \\
& \quad=\Phi\left(x ; t, t_{2}, t_{3}\right) \sum_{w \in \mathfrak{S}_{n}} w\left(x^{-\lambda} \prod_{1 \leqslant i<j \leqslant n} \frac{t x_{i}-x_{j}}{x_{i}-x_{j}}\right) \\
& \quad=v_{\lambda}(t) \Phi\left(x ; t, t_{2}, t_{3}\right) P_{\lambda}\left(x^{-1} ; t\right) .
\end{aligned}
$$

Hence

$$
P_{\lambda}^{\left(\mathrm{BC}_{n}\right)}\left(x ; t, t_{2}, t_{3}\right)=\frac{1}{\left(t_{2} t_{3} ; t\right)_{n-l(\lambda)}} \sum_{\varepsilon \in\{ \pm 1\}^{n}} \Phi\left(x^{\varepsilon} ; t, t_{2}, t_{3}\right) P_{\lambda}\left(x^{-\varepsilon} ; t\right) .
$$

The claim now follows from $P_{m^{n}}(x ; t)=\prod_{i=1}^{n} x_{i}^{m}$ and the fact that $n-l(\lambda)=0$ for $\lambda=m^{n}$ with $m \geqslant 1$.

In the case of $\mathrm{C}_{n}$ we can be brief. As in the $q, t$-case treated earlier, $\left(t, t_{2}\right)=$ $\left(t_{\alpha_{1}}, t_{\alpha_{n}}\right)$ and from the $q=0$ case of (2.50b)

$$
\begin{aligned}
P_{\lambda}^{\left(\mathrm{C}_{n}\right)}\left(x ; t, t_{2}\right) & =P_{\lambda}^{\left(\mathrm{BC}_{n}\right)}\left(x ; t, \pm t_{2}^{1 / 2}\right) \\
& =P_{\lambda}^{\left(\mathrm{C}_{n}, \mathrm{~B}_{n}\right)}\left(x ; 0, t, t_{2}\right)=K_{\lambda}\left(x ; 0, t ; 0,0, \pm t_{2}^{1 / 2}\right) .
\end{aligned}
$$


Accordingly, the $t_{3}=-t_{2}$ case of (2.55) is a one-parameter deformation of the symplectic Schur function [59]

$$
\operatorname{sp}_{2 n, \lambda}(x):=\frac{1}{\Delta_{\mathrm{C}}} \operatorname{det}_{1 \leqslant i, j \leqslant n}\left(x_{i}^{-\lambda_{j}+j-1}-x_{i}^{\lambda_{j}+2 n-j+1}\right) .
$$

The Hall-Littlewood polynomials $P_{\lambda}^{\left(\mathrm{B}_{n}\right)}\left(x ; t, t_{2}\right)$ are given by the $t_{3}=-1$ case of (2.53) where $\lambda$ is now also allowed to be a half-partition. In that case $v_{\lambda}(t)$ is as defined on page 24 but with $\prod_{i \geqslant 0}$ a product over half-integers. By (2.50) we of course also have

$$
P_{\lambda}^{\left(\mathrm{B}_{n}\right)}\left(x ; t, t_{2}\right)=P_{\lambda}^{\left(\mathrm{B}_{n}, \mathrm{~B}_{n}\right)}\left(x ; 0, t, t_{2}\right)=K_{\lambda}\left(x ; 0, t ; t_{2}, 0\right) .
$$

When $t=t_{2}=0$ the $\mathrm{B}_{n}$ Hall-Littlewood polynomials simplify to the odd orthogonal Schur functions [59]

$$
\mathrm{SO}_{2 n+1, \lambda}(x):=\frac{1}{\Delta_{\mathrm{B}}(x)} \operatorname{det}_{1 \leqslant i, j \leqslant n}\left(x_{i}^{-\lambda_{j}+j-1}-x_{i}^{\lambda_{j}+2 n-j}\right),
$$

where

$$
\Delta_{\mathrm{B}}(x):=\prod_{i=1}^{n}\left(1-x_{i}\right) \prod_{1 \leqslant i<j \leqslant n}\left(x_{i}-x_{j}\right)\left(x_{i} x_{j}-1\right)=\operatorname{det}_{1 \leqslant i, j \leqslant n}\left(x_{i}^{j-1}-x_{i}^{2 n-j}\right) .
$$

The $\mathrm{B}_{n}$ analogue of Lemma 2.4 is as follows.

Lemma 2.5. For $m$ a positive integer,

$$
P_{\left(\frac{m}{2}\right)^{n}}^{\left(\mathrm{B}_{n}\right)}\left(x ; t, t_{2}\right)=\sum_{\varepsilon \in\{ \pm 1\}^{n}} \Phi\left(x^{\varepsilon} ; t, t_{2},-1\right) \prod_{i=1}^{n} x_{i}^{-\varepsilon_{i} m / 2}
$$

Finally, the $\mathrm{D}_{n}$ Hall-Littlewood polynomials $P_{\lambda}^{\left(\mathrm{D}_{n}\right)}(x ; t)$ are given by $(2.53)$ with $\left(t_{2}, t_{3}\right)=(-1,1)$ provided we multiply the right side 2 when $l(\lambda)<n$ and $W$ is taken to be the group of even signed-permutations, i.e., $W=\mathfrak{S}_{n} \ltimes$ $(\mathbb{Z} / 2 \mathbb{Z})^{n-1}$. As in the $\mathrm{B}_{n}$ case $\lambda$ can be a partition or half-partition. When $t=0$ this yields the even orthogonal Schur functions (see e.g., [72])

$$
\begin{array}{r}
\mathrm{SO}_{2 n, \lambda}(x):=\frac{1}{2 \Delta_{\mathrm{D}}(x)}\left(\operatorname{det}_{1 \leqslant i, j \leqslant n}\left(x_{i}^{\lambda_{j}+2 n-j-1}+x_{i}^{-\lambda_{j}+j-1}\right)\right. \\
\left.\quad-\operatorname{det}_{1 \leqslant i, j \leqslant n}\left(x_{i}^{\lambda_{j}+2 n-j-1}-x_{i}^{-\lambda_{j}+j-1}\right)\right),
\end{array}
$$

where

$$
\Delta_{\mathrm{D}}(x):=\prod_{1 \leqslant i<j \leqslant n}\left(x_{i}-x_{j}\right)\left(x_{i} x_{j}-1\right)=\frac{1}{2} \operatorname{det}_{1 \leqslant i, j \leqslant n}\left(x_{i}^{j-1}+x_{i}^{2 n-j-1}\right) .
$$

Again we have a simple analogue of Lemma 2.4. 
Lemma 2.6. For $\varepsilon=\left(\varepsilon_{1}, \ldots, \varepsilon_{n}\right) \in\{-1,1\}^{n}$ let $\operatorname{sgn}(\varepsilon):=\prod_{i=1}^{n} \varepsilon_{i}$. Then for $m$ a positive integer

$$
P_{\left(\frac{m}{2}\right)^{n}}^{\left(\mathrm{D}_{n}\right)}(x ; t)=\sum_{\substack{\varepsilon \in\{ \pm 1\}^{n} \\ \operatorname{sgn}(\varepsilon)=1}} \Phi\left(x^{\varepsilon} ; t, 1,-1\right) \prod_{i=1}^{n} x_{i}^{-\varepsilon_{i} m / 2} .
$$

2.7.4. Modified Hall-Littlewood polynomials. The simplest definition of the modified Hall-Littlewood polynomials is as a plethystically substituted ordinary Hall-Littlewood polynomial:

$$
P_{\lambda}^{\prime}(x ; t):=P_{\lambda}\left(\left[\frac{x}{1-t}\right] ; t\right) \quad \text { and } \quad Q_{\lambda}^{\prime}(x ; t):=Q_{\lambda}\left(\left[\frac{x}{1-t}\right] ; t\right),
$$

where $Q_{\lambda}(t)=b_{\lambda}(t) P_{\lambda}(t)$ and

$$
b_{\lambda}(t)=\prod_{i \geqslant 1}(t ; t)_{m_{i}(\lambda)} .
$$

There are numerous other, more insightful, descriptions of the modified polynomials, none of which will be needed in this paper. We nonetheless mention one such description as it highlights the combinatorial nature of the modified polynomials and hence of our character formulas such as (1.4). Let $\operatorname{Tab}(\lambda, \mu)$ be the set of semistandard Young tableaux of shape $\lambda$ and weight (or filling) $\mu$, and let $\mathrm{c}(T)$ be the Lascoux-Schützenberger charge statistic on $\operatorname{Tab}(\lambda, \mu)$ Then [49,64]

$$
Q_{\mu}^{\prime}(t)=\sum_{T \in \operatorname{Tab}(\cdot, \mu)} t^{\mathrm{c}(T)} s_{\text {shape }(T)},
$$

where $s_{\lambda}(x)=\sum_{\operatorname{Tab}(\lambda, \cdot)} x^{T}$ is the Schur function.

\section{Virtual KoORnWinder InTEGRALS}

For $\left\{f_{\lambda}\right\}$ a basis of $\Lambda_{n}, \Lambda$ or $\Lambda^{\mathrm{BC}_{n}}$ and $g$ an arbitrary element of one of these spaces, we write $\left[f_{\lambda}\right] g$ for the coefficient $c_{\lambda}$ in $g=\sum_{\lambda} c_{\lambda} f_{\lambda}$. Although typically $f_{0}=1$ we still write $\left[f_{0}\right] g$ to avoid ambiguity as to the choice of basis.

Our approach to bounded Littlewood identities relies crucially on properties of two linear functionals, denoted $I_{K}$ and $I_{K}^{(n)}$ and referred to as virtual Koornwinder integrals, acting on $\Lambda$ and $\Lambda^{\mathrm{BC}_{n}}$ respectively. Let $f \in \Lambda$. Then the virtual Koornwinder integral $I_{K}$ is defined as [77, page 110; Definition 6]

$$
I_{K}\left(f ; q, t, T ; t_{0}, t_{1}, t_{2}, t_{3}\right):=\left[\tilde{K}_{0}\left(q, t, T ; t_{0}, t_{1}, t_{2}, t_{3}\right)\right] f
$$

where $\tilde{K}_{\lambda}$ is the lifted Koornwinder polynomial. Similarly, for $f \in \Lambda^{\mathrm{BC}_{n}}$ and $x=\left(x_{1}, \ldots, x_{n}\right)$ [77, page 95]

$$
I_{K}^{(n)}\left(f ; q, t ; t_{0}, t_{1}, t_{2}, t_{3}\right):=\left[K_{0}\left(x ; q, t ; t_{0}, t_{1}, t_{2}, t_{3}\right)\right] f .
$$


By (2.32) and $K_{0}=1$ it follows that

$$
\begin{aligned}
I_{K}^{(n)}\left(f ; q, t ; t_{0}, t_{1}, t_{2}, t_{3}\right) & =\frac{\langle 1, f(x)\rangle}{\langle 1,1\rangle} \\
& =\frac{1}{\langle 1,1\rangle} \int_{\mathbb{T}^{n}} \Delta\left(x ; q, t ; t_{0}, t_{1}, t_{2}, t_{3}\right) f(x) \mathrm{d} T(x) .
\end{aligned}
$$

Also, by (2.36), for $f \in \Lambda$ and generic $q, t, t_{0}, t_{1}, t_{2}, t_{3}$

$$
I_{K}^{(n)}\left(f\left(x_{1}^{ \pm}, \ldots, x_{n}^{ \pm} ; q, t ; t_{0}, t_{1}, t_{2}, t_{3}\right)=I_{K}\left(f ; q, t, t^{n} ; t_{0}, t_{1}, t_{2}, t_{3}\right) .\right.
$$

Remark 3.1. Invoking the ring homomorphism $\varphi: \Lambda_{2 n} \rightarrow \Lambda^{\mathrm{BC}_{n}}$ given by

$$
\varphi\left(m_{\lambda}\left(x_{1}, \ldots, x_{2 n}\right)\right)=m_{\lambda}\left(x_{1}^{ \pm}, \ldots, x_{n}^{ \pm 1}\right)
$$

(so that $\operatorname{ker}(\varphi)=\left\langle e_{i}-e_{2 n-i}: 0 \leqslant i<n\right\rangle$ ), it will be convenient to extend $I_{K}^{(n)}$ to also act on $\Lambda_{2 n}$ (or more simply $f \in \Lambda$ ). That is, we set

$$
\begin{aligned}
I_{K}^{(n)}\left(f ; q, t ; t_{0}, t_{1}, t_{2}, t_{3}\right):=\left[K_{0}\left(x ; q, t ; t_{0}, t_{1}, t_{2}, t_{3}\right)\right] & f\left(x_{1}^{ \pm}, \ldots, x_{n}^{ \pm}\right) \\
& f \in \Lambda_{2 n},(\text { or } f \in \Lambda) .
\end{aligned}
$$

Since for symmetric functions

$$
I_{K}^{(n)}\left(f\left(x_{1}, \ldots, x_{2 n}\right) ; q, t ; t_{0}, t_{1}, t_{2}, t_{3}\right)=I_{K}^{(n)}\left(f\left(x_{1}^{ \pm}, \ldots, x_{n}^{ \pm}\right) ; q, t ; t_{0}, t_{1}, t_{2}, t_{3}\right),
$$

we will often not distinguish between (3.2) and (3.5) and simply write

$$
I_{K}^{(n)}\left(f ; q, t ; t_{0}, t_{1}, t_{2}, t_{3}\right) .
$$

The reader is warned that for specialisations that hit the poles (2.35) of the lifted Koornwinder polynomials 3 (3.4) should be treated with great caution as the right-hand side may not be well-defined. In such cases $T$ needs to be specialised before the $t_{r}$ (or at least before some of the $t_{r}$ ). For example, if $T=$ $t^{n}$ and $\left\{t_{0}, t_{1}, t_{2}, t_{3}\right\}=\left\{ \pm 1, \pm t^{1 / 2}\right\}$, then the lifted Koornwinder polynomial $\tilde{K}_{\lambda}$ is ill-defined for all partitions of the form $\left(\lambda_{1}, \ldots, \lambda_{i-1}, 1^{2 n-2 i+2}\right)$ where $\lambda_{1} \geqslant \cdots \geqslant \lambda_{i-1} \geqslant 1$ and $1 \leqslant i \leqslant n+1 / 2$. Accordingly, for (3.4) to hold we must first specialise $T=t^{n}$ before specialising the $t_{r}$. For example, since

$$
\tilde{K}_{1^{2}}\left(q, t, T ; \pm 1, \pm t_{2}\right)=m_{1^{2}}-\frac{(1-T)(T / t+t)\left(t-t_{2}^{2}\right)}{(1-t)(1+t)\left(t-t_{2}^{2}(T / t)^{2}\right)}
$$

(and $\tilde{K}_{0}=1$ ), we have

$$
I_{K}\left(m_{1^{2}} ; q, t, T ; \pm 1, \pm t_{2}\right)=\frac{(1-T)(T / t+t)\left(t-t_{2}^{2}\right)}{(1-t)(1+t)\left(t-t_{2}^{2}(T / t)^{2}\right)} .
$$

\footnotetext{
${ }^{3}$ Since $q^{2-\lambda_{i}-j}$ is a nonpositive integer power of $q$ this can never happen when the product $t_{0} t_{1} t_{2} t_{3}$ contains a positive power of $q$.
} 
This gives $I_{K}\left(m_{1^{2}}\left(q, t, t ; \pm 1, \pm t_{2}\right)=1\right.$ which trivially agrees with

$$
I_{K}^{(1)}\left(m_{1^{2}} ; q, t ; \pm 1, \pm t_{2}\right)=I_{K}^{(1)}\left(1 ; q, t ; \pm 1, \pm t_{2}\right)=1 .
$$

However,

$$
I_{K}\left(m_{1^{2}} ; q, t, T ; \pm 1, \pm t^{1 / 2}\right)=0 .
$$

For specialising in the 'wrong' order we can use [80, Lemma 5.10].

Lemma 3.2. For fixed $n$ let $t_{0} \cdots t_{3} t^{n-2}=t^{k}$ where $k$ is a nonnegative integer. Then

$$
\begin{aligned}
\lim _{T \rightarrow t^{n}} I_{K} & \left(f ; q, t, T ; t_{0}, t_{1}, t_{2}, t_{3}\right) \\
= & \frac{1}{2} I_{K}^{(n)}\left(f ; q, t ; t_{0}, t_{1}, t_{2}, t_{3}\right) \\
& +\frac{1}{2} I_{K}^{(k)}\left(f\left[x_{1}^{ \pm}+\cdots+x_{k}^{ \pm}+\sum_{r=0}^{3} \frac{t_{r}-t / t_{r}}{1-t}\right] ; q, t ; t / t_{0}, t / t_{1}, t / t_{2}, t / t_{3}\right) .
\end{aligned}
$$

Continuing our previous example we find

$$
\begin{aligned}
\lim _{T \rightarrow t} I_{K}(f ; q, t, T ; & \left. \pm 1, \pm t^{1 / 2}\right) \\
& =\frac{1}{2} I_{K}^{(1)}\left(f ; q, t, \pm 1, \pm t^{1 / 2}\right)+\frac{1}{2} I_{K}^{(0)}\left(f( \pm 1) ; q, t, \pm t, \pm t^{1 / 2}\right) .
\end{aligned}
$$

For $f=m_{1^{2}}$, we have $m_{1^{2}}( \pm 1)=-1$ so that this correctly gives $0=\frac{1}{2}+\frac{1}{2}(-1)$.

Lemma 3.3. For $\mu$ a partition and generic $q, t, t_{2}, t_{3}$

$$
I_{K}\left(f[x+\varepsilon] ; q, t, T ;-t,-t^{1 / 2}, t_{2}, t_{3}\right)=I_{K}\left(f ; q, t, t^{1 / 2} T ;-1,-t^{1 / 2}, t_{2}, t_{3}\right) .
$$

Proof. Let $f \in \Lambda$. From definition (3.1) of the virtual Koornwinder integral and the symmetry (2.40) of the lifted Koornwinder polynomials we infer that (see also [77, Equation (7.4) ]4)

$$
\begin{aligned}
I_{K}\left(f\left[x+\frac{t_{0}-t / t_{0}}{1-t}+\frac{t_{1}-t / t_{1}}{1-t}\right] ; q,\right. & \left.t, T ; t / t_{0}, t / t_{1}, t_{2}, t_{3}\right) \\
& =I_{K}\left(f ; q, t, t T / t_{0} t_{1} ; t_{0}, t_{1}, t_{2}, t_{3}\right)
\end{aligned}
$$

If we take $\left\{t_{0}, t_{1}\right\}=\left\{-1,-t^{1 / 2}\right\}=\left\{\varepsilon, \varepsilon t^{1 / 2}\right\}$, so that

$$
\frac{t_{0}-t / t_{0}}{1-t}+\frac{t_{1}-t / t_{1}}{1-t}=\frac{\varepsilon-\varepsilon t}{1-t}+\frac{\varepsilon t^{1 / 2}-\varepsilon t^{1 / 2}}{1-t}=\varepsilon,
$$

this simplifies to (3.6).

\footnotetext{
${ }^{4}$ In [77, Equation (7.4)] the denominator term $(1-t)$ should be corrected to $\left(1-t^{k}\right)$.
} 
In the remainder we consider several closed-form evaluations of virtual Koornwinder integrals over Macdonald polynomials. We begin by recalling two known results:

$$
\begin{aligned}
& I_{K}\left(P_{\mu}(q, t) ; q, t, T ; \pm t^{1 / 2}, \pm(q t)^{1 / 2}\right) \\
= & \chi\left(\mu^{\prime} \text { even }\right) \frac{\left(T^{2} ; q, t^{2}\right)_{\nu}}{\left(q T^{2} / t ; q, t^{2}\right)_{\nu}} \cdot \frac{C_{\nu}^{-}\left(q t ; q, t^{2}\right)}{C_{\nu}^{-}\left(t^{2} ; q, t^{2}\right)}, \quad \nu:=\left(\mu^{\prime} / 2\right)^{\prime}=\left(\mu_{1}, \mu_{3}, \ldots\right),
\end{aligned}
$$

(where $\pm t^{1 / 2}, \pm(q t)^{1 / 2}$ is shorthand for $t^{1 / 2},-t^{1 / 2},(q t)^{1 / 2},-(q t)^{1 / 2}$ ) and

$$
\begin{aligned}
& I_{K}\left(P_{\mu}(q, t) ; q, t, T ;-1,-q^{1 / 2},-t^{1 / 2},-(q t)^{1 / 2}\right) \\
& \quad=(-1)^{|\mu|} \frac{\left(T ; q^{1 / 2}, t^{1 / 2}\right)_{\mu}}{\left(-q^{1 / 2} T / t^{1 / 2} ; q^{1 / 2}, t^{1 / 2}\right)_{\mu}} \cdot \frac{C_{\mu}^{-}\left(-q^{1 / 2} ; q^{1 / 2}, t^{1 / 2}\right)}{C_{\mu}^{-}\left(t^{1 / 2} ; q^{1 / 2}, t^{1 / 2}\right)} .
\end{aligned}
$$

The identity (3.8), which for $T=t^{n}$ is known as the $\mathrm{U}(2 n) / \operatorname{Sp}(2 n)$ vanishing integral, was conjectured in [77, Conjecture 1] and proven in [81, Theorem 4.1]. The identity (3.9) was first stated (up to a trivial sign-change) as the conjectural [79, Equation (5.79)]. By [80, Theorem 8.5], which implies [79, Conjecture Q6], it now also has been proven.

From a symmetry of the virtual Koornwinder polynomials, the virtual Koornwinder integral satisfies the duality [77, Corollary 7.6 $]^{5}$

$$
I_{K}\left(f ; q, t, T ; t_{0}, t_{1}, t_{2}, t_{3}\right)=I_{K}\left(f\left[-\varepsilon\left(\frac{t}{q}\right)^{1 / 2} \frac{1-q}{1-t} x\right] ; t, q, 1 / T ; s_{0}, s_{1}, s_{2}, s_{3}\right),
$$

where $s_{r}=-(q t)^{1 / 2} / t_{r}$. Applying this to (3.8) and then replacing $(q, t, T, \mu)$ by $\left(t, q, 1 / T, \mu^{\prime}\right)$ using (2.4), (2.5b), (2.15) and (2.19) yields the dual integral [81, page 741$]$

$$
I_{K}\left(P_{\mu}(q, t) ; q, t, T ; \pm 1, \pm t^{1 / 2}\right)=\chi(\mu \text { even }) \frac{\left(T^{2} ; q^{2}, t\right)_{\mu / 2}}{\left(q T^{2} / t ; q^{2}, t\right)_{\mu / 2}} \cdot \frac{C_{\mu / 2}^{-}\left(q ; q^{2}, t\right)}{C_{\mu / 2}^{-}\left(t ; q^{2}, t\right)} .
$$

We need two variants of this for $I_{K}^{(n)}$.

For $\lambda$ a partition or half-partition of length at most $n$ define

$$
A_{\lambda}^{(n)}(q, t):=\prod_{1 \leqslant i<j \leqslant n} \frac{\left(q t^{j-i-1}, t^{j-i+1} ; q^{2}\right)_{\lambda_{i}-\lambda_{j}}}{\left(q t^{j-i}, t^{j-i} ; q^{2}\right)_{\lambda_{i}-\lambda_{j}}} .
$$

It is important to note that $A_{\lambda}^{(n)}(q, t)$ depends on the relative differences between the $\lambda_{i}$, and that for $\lambda$ a partition

$$
A_{\lambda}^{(n)}(q, t)=\frac{\left(t^{n} ; q^{2}, t\right)_{\lambda}}{\left(q t^{n-1} ; q^{2}, t\right)_{\lambda}} \cdot \frac{C_{\lambda}^{-}\left(q ; q^{2}, t\right)}{C_{\lambda}^{-}\left(t ; q^{2}, t\right)} .
$$

\footnotetext{
${ }^{5} \operatorname{In}$ [77, Corollary 7.6] $\tilde{\omega}_{q, t}$ should be corrected to $\tilde{\omega}_{t, q}$.
} 
Theorem 3.4. For $\mu$ a partition of length at most $2 n$, let

$$
\tilde{\mu}=\left(\mu_{1}-\mu_{2 n}, \ldots, \mu_{2 n-1}-\mu_{2 n}, 0\right) .
$$

Then

$$
\begin{aligned}
& I_{K}^{(n)}\left(P_{\mu}\left(x_{1}^{ \pm}, \ldots, x_{n}^{ \pm} ; q, t\right) ; q, t ; \pm 1, \pm t^{1 / 2}\right) \\
&=(-1)^{\mu_{2 n}} I_{K}^{(n-1)}\left(P_{\mu}\left(x_{1}^{ \pm}, \ldots, x_{n-1}^{ \pm}, \pm 1 ; q, t\right) ; q, t ; \pm t, \pm t^{1 / 2}\right) \\
&= \begin{cases}A_{\mu / 2}^{(2 n)}(q, t) & \text { if } \tilde{\mu} \text { is even } \\
0 & \text { otherwise, }\end{cases}
\end{aligned}
$$

where $\mu / 2:=\left(\mu_{1} / 2, \mu_{2} / 2, \ldots\right)$.

Theorem 3.5. For $\nu$ a partition of length at most $2 n+1$, let

$$
\tilde{\nu}=\left(\nu_{1}-\nu_{2 n+1}, \ldots, \nu_{2 n}-\nu_{2 n+1}, 0\right) \text {. }
$$

Then

$$
\begin{gathered}
I_{K}^{(n)}\left(P_{\nu}\left(x_{1}^{ \pm}, \ldots, x_{n}^{ \pm}, 1 ; q, t\right) ; q, t ;-1, t, \pm t^{1 / 2}\right) \\
=(-1)^{\nu_{2 n+1}} I_{K}^{(n)}\left(P_{\nu}\left(x_{1}^{ \pm}, \ldots, x_{n}^{ \pm},-1 ; q, t\right) ; q, t ; 1,-t, \pm t^{1 / 2}\right) \\
= \begin{cases}A_{\nu / 2}^{(2 n+1)}(q, t) & \text { if } \tilde{\nu} \text { is even } \\
0 & \text { otherwise. }\end{cases}
\end{gathered}
$$

Proof of Theorem 3.4. From (2.27) we have

$$
\begin{aligned}
P_{\mu}\left(x_{1}^{ \pm}, \ldots, x_{n}^{ \pm} ; q, t\right) & =P_{\tilde{\mu}}\left(x_{1}^{ \pm}, \ldots, x_{n}^{ \pm} ; q, t\right) \\
P_{\mu}\left(x_{1}^{ \pm}, \ldots, x_{n-1}^{ \pm}, \pm 1 ; q, t\right) & =(-1)^{\mu_{2 n}} P_{\tilde{\mu}}\left(x_{1}^{ \pm}, \ldots, x_{n-1}^{ \pm}, \pm 1 ; q, t\right),
\end{aligned}
$$

so that

$$
\begin{aligned}
I_{K}^{(n)}\left(P_{\mu}\left(x_{1}^{ \pm}, \ldots, x_{n}^{ \pm} ; q, t\right)\right. & \left.; q, t ; t_{0}, t_{1}, t_{2}, t_{3}\right) \\
& =I_{K}^{(n)}\left(P_{\tilde{\mu}}\left(x_{1}^{ \pm}, \ldots, x_{n}^{ \pm} ; q, t\right) ; q, t ; t_{0}, t_{1}, t_{2}, t_{3}\right)
\end{aligned}
$$

and

$$
\begin{aligned}
& I_{K}^{(n-1)}\left(P_{\mu}\left(x_{1}^{ \pm}, \ldots, x_{n-1}^{ \pm}, \pm 1 ; q, t\right) ; q, t ; t_{0}, t_{1}, t_{2}, t_{3}\right) \\
& \quad=(-1)^{\mu_{2 n}} I_{K}^{(n-1)}\left(P_{\tilde{\mu}}\left(x_{1}^{ \pm}, \ldots, x_{n-1}^{ \pm}, \pm 1 ; q, t\right) ; q, t ; t_{0}, t_{1}, t_{2}, t_{3}\right)
\end{aligned}
$$

Since $A_{\mu / 2}^{(2 n)}(q, t)=A_{\tilde{\mu} / 2}^{(2 n)}(q, t)$, it thus suffices to prove that

$$
\begin{aligned}
& \quad I_{K}^{(n)}\left(P_{\omega}\left(x_{1}^{ \pm}, \ldots, x_{n}^{ \pm} ; q, t\right) ; q, t ; \pm 1, \pm t^{1 / 2}\right) \\
& =I_{K}^{(n-1)}\left(P_{\omega}\left(x_{1}^{ \pm}, \ldots, x_{n-1}^{ \pm}, \pm 1 ; q, t\right) ; q, t ; \pm 1, \pm t^{1 / 2}\right)=\chi(\omega \text { even }) A_{\omega / 2}^{(2 n)}(q, t),
\end{aligned}
$$

for $\omega$ a partition such that $l(\omega)<2 n$. 
Plethystically, for $\left\{t_{0}, t_{1}, t_{2}, t_{3}\right\}=\left\{ \pm 1, \pm t^{1 / 2}\right\}=\left\{1, \varepsilon, t^{1 / 2}, \varepsilon t^{1 / 2}\right\}$ we have

$$
\sum_{r=0}^{3} \frac{t_{r}-t / t_{r}}{1-t}=\frac{1-t}{1-t}+\frac{\varepsilon-\varepsilon t}{1-t}+\frac{t^{1 / 2}-t^{1 / 2}}{1-t}+\frac{\varepsilon t^{1 / 2}-\varepsilon t^{1 / 2}}{1-t}=1+\varepsilon .
$$

Hence, from Lemma 3.2 with $\left\{t_{0}, t_{1}, t_{2}, t_{3}\right\}=\left\{ \pm 1, \pm t^{1 / 2}\right\}$ and $f=P_{\mu}(q, t)$,

$$
\begin{aligned}
\lim _{T \rightarrow t^{n}} I_{K} & \left(P_{\mu}(q, t), q, t, T ; \pm 1, \pm t^{1 / 2}\right) \\
= & \frac{1}{2} I_{K}^{(n)}\left(P_{\mu}\left(x_{1}^{ \pm}, \ldots, x_{n}^{ \pm} ; q, t\right) ; q, t ; \pm 1, \pm t^{1 / 2}\right) \\
& +\frac{1}{2} I_{K}^{(n-1)}\left(P_{\mu}\left(x_{1}^{ \pm}, \ldots, x_{n-1}^{ \pm}, \pm 1 ; q, t\right) ; q, t ; \pm t, \pm t^{1 / 2}\right) .
\end{aligned}
$$

By (3.10) and (3.12) the left-hand side is equal to $\chi(\mu$ even $) A_{\mu / 2}^{(2 n)}(q, t)$, which implies

$$
\begin{aligned}
& \frac{1}{2} I_{K}^{(n)}\left(P_{\mu}\left(x_{1}^{ \pm}, \ldots, x_{n}^{ \pm} ; q, t\right) ; q, t ; \pm 1, \pm t^{1 / 2}\right) \\
& \quad+\frac{1}{2} I_{K}^{(n-1)}\left(P_{\mu}\left(x_{1}^{ \pm}, \ldots, x_{n-1}^{ \pm}, \pm 1 ; q, t\right) ; q, t ; \pm t, \pm t^{1 / 2}\right)=\chi(\mu \text { even }) A_{\mu / 2}^{(2 n)}(q, t) .
\end{aligned}
$$

Again using (3.13) as well as

$$
\chi(\mu \text { even }) A_{\mu / 2}^{(2 n)}(q, t)=\chi\left(\mu_{2 n} \text { even }\right) \chi(\tilde{\mu} \text { even }) A_{\tilde{\mu} / 2}^{(2 n)}(q, t),
$$

and then renaming $\tilde{\mu}_{i}=\mu_{i}-\mu_{2 n}$ as $\omega_{i}$ for $1 \leqslant i \leqslant 2 n-1$, and $\mu_{2 n}$ as $k$, it follows that

$$
\begin{aligned}
& \frac{1}{2} I_{K}^{(n)}\left(P_{\omega}\left(x_{1}^{ \pm}, \ldots, x_{n}^{ \pm} ; q, t\right) ; q, t ; \pm 1, \pm t^{1 / 2}\right) \\
& +\frac{1}{2}(-1)^{k} I_{K}^{(n-1)}\left(P_{\omega}\left(x_{1}^{ \pm}, \ldots, x_{n-1}^{ \pm}, \pm 1 ; q, t\right) ; q, t ; \pm t, \pm t^{1 / 2}\right) \\
& \quad=\chi(k \text { even }) \chi(\omega \text { even }) A_{\omega / 2}^{(2 n)}(q, t)
\end{aligned}
$$

for $\omega$ a partition of length at most $2 n-1$ and $k$ an integer. This implies (3.14), completing the proof.

Proof of Theorem 3.5. Let $z \in\{-1,1\}$. Since

$$
\begin{aligned}
I_{K}^{(n)}\left(P_{\nu}\left(x_{1}^{ \pm}, \ldots, x_{n}^{ \pm}, z ; q, t\right) ; q, t ; t_{0}, t_{1}, t_{2}, t_{3}\right) & \\
& =z^{\nu_{2 n+1}} I_{K}^{(n)}\left(P_{\tilde{\nu}}\left(x_{1}^{ \pm}, \ldots, x_{n}^{ \pm}, z ; q, t\right) ; q, t ; t_{0}, t_{1}, t_{2}, t_{3}\right)
\end{aligned}
$$

and $A_{\nu / 2}^{(2 n+1)}(q, t)=A_{\tilde{\nu} / 2}^{(2 n+1)}(q, t)$, it is enough to show that

$$
I_{K}^{(n)}\left(P_{\tau}\left(x_{1}^{ \pm}, \ldots, x_{n}^{ \pm}, z ; q, t\right) ; q, t ;-z, z t, \pm t^{1 / 2}\right)=\chi(\tau \text { even }) A_{\tau / 2}^{(2 n+1)}(q, t)
$$

for $\tau$ a partition such that $l(\tau) \leqslant 2 n$. Since $\left\{t_{0}, t_{1}, t_{2}, t_{3}\right\}=\left\{1, \varepsilon t, t^{1 / 2}, \varepsilon t^{1 / 2}\right\}$ we have $\sum_{r=0}^{3}\left(t_{r}-t / t_{r}\right) /(1-t)=1-\varepsilon$. Lemma 3.2 with $f(x)=P_{\nu}([x+\varepsilon] ; q, t)$ 
thus implies that

$$
\begin{aligned}
\lim _{T \rightarrow t^{n}} I_{K}\left(P_{\nu}([x+\varepsilon] ; q, t), q, t, T ; 1,-t, \pm t^{1 / 2}\right) \\
\quad=\frac{1}{2} \sum_{z \in\{-1,1\}} I_{K}^{(n)}\left(P_{\nu}\left(x_{1}^{ \pm}, \ldots, x_{n}^{ \pm}, z ; q, t\right) ; q, t ;-z, z t, \pm t^{1 / 2}\right) .
\end{aligned}
$$

Recalling Lemma 3.3, the expression on the left equates to

$$
\lim _{T \rightarrow t^{n}} I_{K}\left(P_{\nu}(q, t), q, t, t^{1 / 2} T ; \pm 1, \pm t^{1 / 2}\right),
$$

which by (3.10) and (3.12) evaluates to $\chi\left(\nu\right.$ even) $A_{\nu / 2}^{(2 n+1)}(q, t)$. Putting this all together yields

$$
\begin{aligned}
\frac{1}{2} \sum_{z \in\{-1,1\}} I_{K}^{(n)}\left(P_{\nu}\left(x_{1}^{ \pm}, \ldots, x_{n}^{ \pm}, z ; q, t\right) ; q, t ;-z, z t, \pm t^{1 / 2}\right) & \\
& =\chi(\nu \text { even }) A_{\nu / 2}^{(2 n+1)}(q, t),
\end{aligned}
$$

which by (3.15) can also be written as

$$
\begin{aligned}
\frac{1}{2} \sum_{z \in\{-1,1\}} z^{k} I_{K}^{(n)}\left(P_{\tau}\left(x_{1}^{ \pm}, \ldots, x_{n}^{ \pm}, z ; q, t\right) ; q\right. & \left.t ;-z, z t, \pm t^{1 / 2}\right) \\
& =\chi(k \text { even }) \chi(\tau \text { even }) A_{\tau / 2}^{(2 n+1)}(q, t),
\end{aligned}
$$

for $\tau$ a partition of length at most $2 n$ and $k$ an integer. This implies (3.16).

Theorem 3.6. We have

$$
I_{K}\left(P_{\mu}(q, t) ; q, t, T ;-1, q, \pm t^{1 / 2}\right)=(-1)^{|\mu|} \frac{\left(T^{2} ; q^{2}, t\right)_{\lceil\mu / 2\rceil}}{\left(q T^{2} / t ; q^{2}, t\right)_{\lceil\mu / 2\rceil}} \cdot \frac{1}{b_{\mu}^{\mathrm{ea}}(q, t)}
$$

Proof. From (3.3), definition (2.31) of the Koornwinder density and Gustafson's integral (2.33) it follows that

$$
\begin{aligned}
& I_{K}^{(n)}\left(f ; q, t ; q t_{0}, t_{1}, t_{2}, t_{3}\right) \\
& =I_{K}^{(n)}\left(f\left(x_{1}^{ \pm}, \ldots, x_{n}^{ \pm}\right) \prod_{i=1}^{n}\left(1-t_{0} x_{i}^{ \pm}\right) ; q, t ; t_{0}, t_{1}, t_{2}, t_{3}\right) \prod_{i=1}^{n} \frac{1-t_{0} t_{1} t_{2} t_{3} t^{n+i-2}}{\prod_{r=1}^{3}\left(1-t_{0} t_{r} t^{i-1}\right)}
\end{aligned}
$$

If $f=P_{\mu}(q, t)$ we can use the $e$-Pieri rule (2.23) to expand the integrand. Hence

$$
\begin{aligned}
& I_{K}^{(n)}\left(P_{\mu}(q, t) ; q, t ; q t_{0},\right.\left.t_{1}, t_{2}, t_{3}\right)=\prod_{i=1}^{n} \frac{1-t_{0} t_{1} t_{2} t_{3} t^{n+i-2}}{\prod_{r=1}^{3}\left(1-t_{0} t_{r} t^{i-1}\right)} \\
& \times \sum_{\lambda \supset \mu}\left(-t_{0}\right)^{|\lambda / \mu|} \psi_{\lambda / \mu}^{\prime}(q, t) I_{K}^{(n)}\left(P_{\lambda}(q, t) ; q, t ; t_{0}, t_{1}, t_{2}, t_{3}\right) .
\end{aligned}
$$


For $\left(t_{0}, t_{1}, t_{2}, t_{3}\right)=\left(1,-1, t^{1 / 2},-t^{1 / 2}\right)$ this yields

$$
\begin{aligned}
I_{K}^{(n)}\left(P_{\mu}(q, t) ; q, t ; q,-\right. & \left.1, \pm t^{1 / 2}\right) \\
& =\frac{1}{2} \sum_{\lambda \supset \mu}(-1)^{|\lambda / \mu|} \psi_{\lambda / \mu}^{\prime}(q, t) I_{K}^{(n)}\left(P_{\lambda}(q, t) ; q, t ; \pm 1, \pm t^{1 / 2}\right) .
\end{aligned}
$$

The integral in the summand evaluates in closed form by Theorem [3.4. In particular it vanishes unless (i) $\lambda$ is even or (ii) $\lambda$ is odd and $l(\lambda)=2 n$. Since $\psi_{\lambda / \mu}^{\prime}(q, t)$ is zero unless $\lambda / \mu$ is a vertical strip, this fixes $\lambda$ as $\lambda=2\lceil\mu / 2\rceil=: \nu$ in case (i) and $\lambda=2\lfloor\mu / 2\rfloor+1^{2 n}=: \omega$ in case (ii). Noting the three congruences

$$
|\nu| \equiv|\omega| \equiv 0 \quad(\bmod 2), \quad|\nu / \mu|=\operatorname{odd}(\mu) \equiv|\mu| \quad(\bmod 2),
$$

and

$$
|\omega / \mu|=2 n-\operatorname{odd}(\mu) \equiv|\mu| \quad(\bmod 2)
$$

we obtain

$$
\begin{aligned}
I_{K}^{(n)}\left(P_{\mu}(q, t) ; q, t ; q,\right. & \left.-1, \pm t^{1 / 2}\right) \\
& =\frac{1}{2}(-1)^{|\mu|}\left(\psi_{\nu / \mu}^{\prime}(q, t) A_{\nu / 2}^{(2 n)}(q, t)+\psi_{\omega / \mu}^{\prime}(q, t) A_{\omega / 2}^{(2 n)}(q, t)\right) .
\end{aligned}
$$

We will now show that both terms on the right are the same, resulting in

$$
I_{K}^{(n)}\left(P_{\mu}(q, t) ; q, t ; q,-1, \pm t^{1 / 2}\right)=(-1)^{|\mu|} \psi_{\nu / \mu}^{\prime}(q, t) A_{\nu / 2}^{(2 n)}(q, t) .
$$

First we note that since $\omega / 2=\lfloor\mu / 2\rfloor+\left(\frac{1}{2}\right)^{2 n}$ and $A_{\mu}^{(n)}(q, t)$ depends on the relative differences of the $\mu_{i}$, we have

$$
A_{\omega / 2}^{(2 n)}(q, t)=A_{\lfloor\mu / 2\rfloor}^{(2 n)}(q, t) .
$$

Moreover, by (3.11) and $\nu / 2=\lceil\mu / 2\rceil$,

$$
\begin{gathered}
A_{\lfloor\mu / 2\rfloor}^{(2 n)}(q, t)=A_{\nu / 2}^{(2 n)}(q, t) \prod_{\substack{1 \leqslant i<j \leqslant 2 n \\
\mu_{i} \text { odd, } \mu_{j} \text { even }}} \frac{1-q^{\mu_{i}-\mu_{j}} t^{j-i}}{1-q^{\mu_{i}-\mu_{j}} t^{j-i-1}} \cdot \frac{1-q^{\mu_{i}-\mu_{j}-1} t^{j-i}}{1-q^{\mu_{i}-\mu_{j}-1} t^{j-i+1}} \\
\times \prod_{\substack{1 \leqslant i<j \leqslant 2 n \\
\mu_{i} \text { even, } \mu_{j} \text { odd }}} \frac{1-q^{\mu_{i}-\mu_{j}} t^{j-i-1}}{1-q^{\mu_{i}-\mu_{j} t^{j-i}}} \cdot \frac{1-q^{\mu_{i}-\mu_{j}-1} t^{j-i+1}}{1-q^{\mu_{i}-\mu_{j}-1} t^{j-i}} .
\end{gathered}
$$

But from (2.24) it follows that

$$
\psi_{\nu / \mu}^{\prime}(q, t)=\prod_{\substack{1 \leqslant i<j \leqslant 2 n \\ \mu_{i} \text { even, } \mu_{j} \text { odd }}} \frac{1-q^{\mu_{i}-\mu_{j}} t^{j-i-1}}{1-q^{\mu_{i}-\mu_{j}} t^{j-i}} \cdot \frac{1-q^{\mu_{i}-\mu_{j}-1} t^{j-i+1}}{1-q^{\mu_{i}-\mu_{j}-1} t^{j-i}}
$$


and

$$
\psi_{\omega / \mu}^{\prime}(q, t)=\prod_{\substack{1 \leqslant i<j \leqslant 2 n \\ \mu_{i} \text { odd }, \mu_{j} \text { even }}} \frac{1-q^{\mu_{i}-\mu_{j}} t^{j-i-1}}{1-q^{\mu_{i}-\mu_{j}} t^{j-i}} \cdot \frac{1-q^{\mu_{i}-\mu_{j}-1} t^{j-i+1}}{1-q^{\mu_{i}-\mu_{j}-1} t^{j-i}},
$$

so that

$$
\psi_{\omega / \mu}^{\prime}(q, t) A_{\omega / 2}^{(2 n)}(q, t)=\psi_{\nu / \mu}^{\prime}(q, t) A_{\nu / 2}^{(2 n)}(q, t),
$$

establishing (3.18).

Since $\nu=2\lceil\mu / 2\rceil$ is even we can use (3.12) to write the right side of (3.18) as

$$
(-1)^{|\mu|} \psi_{\nu / \mu}^{\prime}(q, t) \frac{\left(t^{2 n} ; q^{2}, t\right)_{\nu / 2}}{\left(q t^{2 n-1} ; q^{2}, t\right)_{\nu / 2}} \cdot \frac{C_{\nu / 2}^{-}\left(q ; q^{2}, t\right)}{C_{\nu / 2}^{-}\left(t ; q^{2}, t\right)} .
$$

By Lemma 2.1 this is also

$$
(-1)^{|\mu|} \frac{\left(t^{2 n} ; q^{2}, t\right)_{\nu / 2}}{\left(q t^{2 n-1} ; q^{2}, t\right)_{\nu / 2}} \cdot \frac{1}{b_{\mu}^{\mathrm{ea}}(q, t)}
$$

Hence

$$
I_{K}^{(n)}\left(P_{\mu}(q, t) ; q, t ;-1, q, \pm t^{1 / 2}\right)=(-1)^{|\mu|} \frac{\left(t^{2 n} ; q^{2}, t\right)_{\lceil\mu / 2\rceil}}{\left(q t^{2 n-1} ; q^{2}, t\right)_{\lceil\mu / 2\rceil}} \cdot \frac{1}{b_{\mu}^{\mathrm{ea}}(q, t)} .
$$

Since both sides vanish if $l(\mu)>2 n$ this holds for all partitions $\mu$.

For fixed $\mu$

$$
I_{K}\left(P_{\mu}(q, t) ; q, t, T ; t_{0}, t_{1}, t_{2}, t_{3}\right)
$$

is a rational function in $T$. By (3.4) and (3.19), equation (3.17) holds for $T=t^{n}$ for all nonnegative integers $n$. Hence it holds for arbitrary $T$.

Our final virtual Koornwinder integral involves the Rogers-Szegö polynomial (2.8) and does not appear to have a simple analogue at the $q, t$-level.

Theorem 3.7. For $\mu$ a partition of length at most $2 n$,

$$
\begin{aligned}
& I_{K}^{(n)}\left(P_{\mu}(q, 0) ; q, 0 ; 0,0, t_{2}, t_{3}\right) \\
& :=\lim _{t \rightarrow 0} I_{K}^{(n)}\left(P_{\mu}(q, t) ; q, t ; 0,0, t_{2}, t_{3}\right)=h_{\mu^{\prime}}^{(2 n)}\left(-t_{2},-t_{3} ; q\right) .
\end{aligned}
$$

Proof of Theorem 3.7. Let $x=\left(x_{1}, \ldots, x_{n}\right)$. By (3.2) equation (3.20) may also be stated as the rational function identity

$$
\left[K_{0}\left(x ; q, 0 ; 0,0, t_{2}, t_{3}\right)\right] P_{\mu}\left(x^{ \pm}, q, 0\right)=h_{\mu^{\prime}}^{(2 n)}\left(-t_{2},-t_{3} ; q\right) .
$$

Without loss of generality we may thus assume that $\left|t_{2}\right|,\left|t_{3}\right|<1$ below.

Noting that

$$
h_{\lambda}^{(2 n)}(0,0 ; q)=\chi(\lambda \text { even })
$$


the $t_{2}=t_{3}=0$ case of (3.20), viz.

$$
I_{K}^{(n)}\left(P_{\mu}(q, 0) ; q, 0 ; 0,0,0,0\right)=\chi\left(\mu^{\prime} \text { even }\right),
$$

follows from (3.8) (with $T=t^{n}$ ) in the $t \rightarrow 0$ limit.

To include the parameter $t_{2}$ we use that (see (2.33)

$$
\langle 1,1\rangle_{q, 0 ; 0,0, t_{2}, 0}^{(n)}=\langle 1,1\rangle_{q, 0 ; 0,0,0,0}^{(n)}
$$

and

$$
\Delta\left(x ; q, 0 ; 0,0, t_{2}, 0\right)=\Delta(x ; q, 0 ; 0,0,0,0) \prod_{i=1}^{n} \frac{1}{\left(t_{2} x_{i}^{ \pm} ; q\right)_{\infty}} .
$$

From (3.3) it thus follows that

$$
\begin{aligned}
f_{\mu}\left(t_{2} ; q\right) & :=I_{K}^{(n)}\left(P_{\mu}(q, 0) ; q, 0 ; 0,0, t_{2}, 0\right) \\
& =I_{K}^{(n)}\left(P_{\mu}\left(x_{1}^{ \pm}, \ldots, x_{n}^{ \pm} ; q, 0\right) \prod_{i=1}^{2 n} \frac{1}{\left(t_{2} x_{i}^{ \pm} ; q\right)_{\infty}} ; q, 0 ; 0,0,0,0\right) .
\end{aligned}
$$

By the $g$-Pieri rule (2.21) for $t=0$ this yields

$$
\begin{aligned}
f_{\mu}\left(t_{2} ; q\right) & =\sum_{\nu \supset \mu} t_{2}^{|\nu / \mu|} \varphi_{\nu / \mu}(q, 0) I_{K}^{(n)}\left(P_{\nu}(q, 0) ; q, 0 ; 0,0,0,0\right) \\
& =\sum_{\substack{\nu \supset \mu \\
\nu^{\prime} \text { even } \\
l(\nu) \leqslant 2 n}} t_{2}^{|\nu / \mu|} \varphi_{\nu / \mu}(q, 0)
\end{aligned}
$$

where the second equality follows from (3.21). Since $\varphi_{\nu / \mu}(q, 0)$ is zero unless $\nu / \mu$ is a horizontal strip and $\nu^{\prime}$ must be even, this fixes $\nu$ as $\nu_{2 i-1}=\nu_{2 i}=\mu_{2 i-1}$ for $1 \leqslant i \leqslant n$. This is equivalent to $\nu_{i}^{\prime}=\mu_{i}^{\prime}+\chi\left(\mu_{i}^{\prime}\right.$ odd $)$, so that $|\nu / \mu|$ is given by the number of odd parts of $\mu^{\prime}$, i.e., by odd $\left(\mu^{\prime}\right)$. Hence

$$
f_{\mu}\left(t_{2} ; q\right)=t_{2}^{\text {odd }\left(\mu^{\prime}\right)} \varphi_{\nu / \mu}(q, 0)
$$

with $\nu$ fixed as above. From the expression for $\varphi_{\lambda / \mu}(q, t)$ as given in (2.22) it follows that 6

$$
\varphi_{\nu / \mu}(q, 0)=\prod_{i \geqslant 1} \frac{(q ; q)_{\mu_{i}-\mu_{i+1}}}{(q ; q)_{\nu_{i}-\mu_{i}}(q ; q)_{\mu_{i}-\nu_{i+1}}}=\frac{1}{(q ; q)_{\nu_{1}-\mu_{1}}} \prod_{i \geqslant 1}\left[\begin{array}{l}
\mu_{i}-\mu_{i+1} \\
\mu_{i}-\nu_{i+1}
\end{array}\right]_{q} .
$$

When $\nu_{2 i-1}=\nu_{2 i}=\mu_{2 i-1}$ this simplifies to $\varphi_{\nu / \mu}(q, 0)=1$, since either $\nu_{i+1}=\mu_{i}$ or $\nu_{i+1}=\mu_{i+1}$. Hence

$$
f_{\mu}(a ; q)=t_{2}^{\mathrm{odd}\left(\mu^{\prime}\right)}
$$

\footnotetext{
${ }^{6}$ Alternatively, this follows from the Pieri coefficient $\varphi_{\lambda / \mu}^{\prime}(t)$ for Hall-Littlewood polynomials, thanks to $\varphi_{\nu / \mu}(q, 0)=\varphi_{\nu^{\prime} / \mu^{\prime}}^{\prime}(0, q)=\varphi_{\nu^{\prime} / \mu^{\prime}}^{\prime}(q)$.
} 
To also include the parameter $b$ we proceed in almost identical fashion. By

$$
\langle 1,1\rangle_{q, 0 ; 0,0, t_{2}, t_{3}}^{(n)}=\frac{1}{\left(t_{2} t_{3} ; q\right)_{\infty}}\langle 1,1\rangle_{q, 0 ; 0,0, t_{2}, 0}^{(n)}
$$

and

$$
\Delta\left(x ; 0,0, t_{2}, t_{3} ; q, 0\right)=\Delta\left(x ; 0,0, t_{2}, 0 ; q, 0\right) \prod_{i=1}^{n} \frac{1}{\left(t_{2} x_{i}^{ \pm} ; q\right)_{\infty}}
$$

and following previous steps, we obtain

$$
\begin{aligned}
f_{\mu}\left(t_{2}, t_{3} ; q\right) & :=I_{K}^{(n)}\left(P_{\mu}(q, 0) ; q, 0 ; 0,0, t_{2}, t_{3}\right) \\
& =\left(t_{2} t_{3} ; q\right)_{\infty} \sum_{\nu \supset \mu} t_{2}^{|\nu / \mu|} f_{\nu}\left(t_{2} ; q\right) \varphi_{\nu / \mu}(q, 0) \\
& =\left(t_{2} t_{3} ; q\right)_{\infty} \sum_{\substack{\nu \supset \mu \\
l(\nu) \leqslant 2 n}} t_{2}^{\operatorname{odd}\left(\nu^{\prime}\right)} t_{3}^{|\nu / \mu|} \varphi_{\nu / \mu}(q, 0) .
\end{aligned}
$$

Here the second line uses the definition of $f_{\nu}\left(t_{2} ; q\right)$ as given in (3.22) and the third line its evaluation (3.24). To complete the proof we write $\nu_{i}=\mu_{i}+k_{i}$ for $1 \leqslant i \leqslant 2 n$, and note that (see [96, page 822])

$$
\operatorname{odd}\left(\nu^{\prime}\right)=\operatorname{odd}\left(\mu^{\prime}\right)+\sum_{i=1}^{2 n}(-1)^{i+1} k_{i}
$$

Once again using (3.23) we get

$$
f_{\mu}\left(t_{2}, t_{3} ; q\right)=\left(t_{2} t_{3} ; q\right)_{\infty} t_{2}^{\operatorname{odd}\left(\mu^{\prime}\right)} \sum_{k_{1}, \ldots, k_{2 n} \geqslant 0} \frac{1}{(q ; q)_{k_{1}}} \prod_{i \geqslant 1} t_{2}^{(-1)^{i+1} k_{i}} t_{3}^{k_{i}}\left[\begin{array}{c}
\mu_{i}-\mu_{i+1} \\
k_{i+1}
\end{array}\right]_{q} .
$$

Summing over $k_{1}$ by [27, Equation (II.1)]

$$
\sum_{k \geqslant 0} \frac{z^{k}}{(q ; q)_{k}}=\frac{1}{(z ; q)_{\infty}} \quad \text { for }|z|<1
$$

and recalling definition (2.7), we finally obtain

$$
\begin{aligned}
f_{\mu}\left(t_{2}, t_{3} ; q\right) & =t_{2}^{\text {odd }\left(\mu^{\prime}\right)} \prod_{\substack{i=1 \\
i \text { odd }}}^{2 n-1} H_{m_{i}\left(\mu^{\prime}\right)}\left(t_{3} / t_{2} ; q\right) \prod_{\substack{i=1 \\
i \text { even }}}^{2 n-1} H_{m_{i}\left(\mu^{\prime}\right)}\left(t_{2} t_{3} ; q\right) \\
& =h_{\mu^{\prime}}^{(2 n)}\left(-t_{2},-t_{3} ; q\right) .
\end{aligned}
$$




\section{Bounded Littlewood identities}

In this section, which is at the heart of the paper, we use MacdonaldKoornwinder theory and virtual Koornwinder integrals in particular to prove bounded Littlewood identities for Macdonald and Hall-Littlewood polynomials.

\subsection{Statement of results.}

4.1.1. q,t-Identities. There are five known Littlewood identities for Macdonald polynomials, the first four of which are due to Macdonald [64, page 349]. By introducing an additional parameter $a$, they may be written as the pair [96. Proposition 1.3]

$$
\sum_{\lambda} a^{\text {odd }(\lambda)} b_{\lambda}^{\text {oa }}(q, t) P_{\lambda}(x ; q, t)=\prod_{i=1}^{n} \frac{\left(1+a x_{i}\right)\left(q t x_{i}^{2} ; q^{2}\right)_{\infty}}{\left(x_{i}^{2} ; q^{2}\right)_{\infty}} \prod_{1 \leqslant i<j \leqslant n} \frac{\left(t x_{i} x_{j} ; q\right)_{\infty}}{\left(x_{i} x_{j} ; q\right)_{\infty}}
$$

and

$$
\sum_{\lambda} a^{\operatorname{odd}\left(\lambda^{\prime}\right)} b_{\lambda}^{\mathrm{el}}(q, t) P_{\lambda}(x ; q, t)=\prod_{i=1}^{n} \frac{\left(a t x_{i} ; q\right)_{\infty}}{\left(a x_{i} ; q\right)_{\infty}} \prod_{1 \leqslant i<j \leqslant n} \frac{\left(t x_{i} x_{j} ; q\right)_{\infty}}{\left(x_{i} x_{j} ; q\right)_{\infty}},
$$

with Macdonald's identities corresponding to $a=0$ and $a=1$. Here

$$
b_{\lambda}^{\mathrm{oa}}(q, t):=\prod_{\substack{s \in \lambda \\ a(s) \text { odd }}} b_{\lambda}(s ; q, t) \quad \text { and } \quad b_{\lambda}^{\mathrm{el}}(q, t):=\prod_{\substack{s \in \lambda \\ l(s) \text { even }}} b_{\lambda}(s ; q, t),
$$

to be compared with (2.3) and (2.26). The fifth identity was conjectured by Kawanaka [42] and subsequently proven in [47] (see also [79]):

$$
\sum_{\lambda} b_{\lambda}^{-}(q, t) P_{\lambda}\left(x ; q^{2}, t^{2}\right)=\prod_{i=1}^{n} \frac{\left(-t x_{i} ; q\right)_{\infty}}{\left(x_{i} ; q\right)_{\infty}} \prod_{1 \leqslant i<j \leqslant n} \frac{\left(t^{2} x_{i} x_{j} ; q^{2}\right)_{\infty}}{\left(x_{i} x_{j} ; q^{2}\right)_{\infty}},
$$

where

$$
b_{\lambda}^{-}(q, t):=\prod_{s \in \lambda} \frac{1+q^{a(s)} t^{l(s)+1}}{1-q^{a(s)+1} t^{l(s)}} .
$$

In the following we generalise all of (4.1)-(4.3)

For $m$ a nonnegative integer and $\lambda$ a partition let

$$
b_{\lambda ; m}^{\mathrm{oa}}(q, t):=b_{\lambda}^{\mathrm{oa}}(q, t) \prod_{\substack{s \in \lambda \\ a^{\prime}(s) \text { odd }}} \frac{1-q^{2 m-a^{\prime}(s)+1} t^{l^{\prime}(s)}}{1-q^{2 m-a^{\prime}(s)} t^{l^{\prime}(s)+1}} .
$$


Note that $b_{\lambda ; m}^{\text {oa }}(q, t)=0$ if $\lambda_{1}>2 m+1$ and that for $\lambda$ an even partition

$$
b_{\lambda ; m}^{\mathrm{oa}}(q, t)=b_{\lambda}^{\mathrm{oa}}(q, t) \prod_{\substack{s \in \lambda \\ a^{\prime}(s) \text { even }}} \frac{1-q^{2 m-a^{\prime}(s)} t^{l^{\prime}(s)}}{1-q^{2 m-a^{\prime}(s)-1} t^{l^{\prime}(s)+1}} .
$$

Our first bounded Littlewood identity generalises (4.1).

Theorem 4.1. For $x=\left(x_{1}, \ldots, x_{n}\right)$ and $m$ a nonnegative integer,

$$
\sum_{\lambda} a^{\text {odd }(\lambda)} b_{\lambda ; m}^{\mathrm{oa}}(q, t) P_{\lambda}(x ; q, t)=\left(\prod_{i=1}^{n} x_{i}^{m}\left(1+a x_{i}\right)\right) P_{m^{n}}^{\left(\mathrm{C}_{n}, \mathrm{~B}_{n}\right)}(x ; q, t, q t) .
$$

Macdonald's identity (4.1) follows in the large- $m$ limit by application of (2.38) and (2.47).

When $a=0$ the summand on the left vanishes unless $\lambda$ is even so that 7

$$
\sum_{\lambda \text { even }} b_{\lambda ; m}^{\text {oa }}(q, t) P_{\lambda}(x ; q, t)=\left(x_{1} \cdots x_{n}\right)^{m} P_{m^{n}}^{\left(\mathrm{C}_{n}, \mathrm{~B}_{n}\right)}(x ; q, t, q t) .
$$

This is a $q, t$-analogue of the Désarménien-Proctor-Stembridge determinant formula [19, 76, 88]

$$
\sum_{\substack{\lambda \text { even } \\ \lambda_{1} \leqslant 2 m}} s_{\lambda}(x)=\frac{\operatorname{det}_{1 \leqslant i, j \leqslant n}\left(x_{i}^{j-1}-x_{i}^{2 m+2 n-j+1}\right)}{\prod_{i=1}^{n}\left(1-x_{i}^{2}\right) \prod_{1 \leqslant i<j \leqslant n}\left(x_{i}-x_{j}\right)\left(x_{i} x_{j}-1\right)},
$$

which expresses the symplectic Schur function $\operatorname{sp}_{2 n, m^{n}}(x)$ (times $\left.\left(x_{1} \cdots x_{n}\right)^{m}\right)$ in terms of Schur functions. Equivalently, (4.6) is a branching formula for the character of the symplectic group $\operatorname{Sp}(n, \mathbb{C})$ indexed by $m \Lambda_{n}$ in terms of characters of the general linear group $\operatorname{GL}(n, \mathbb{C})$. Like (1.2), the determinant (4.6) is important in the theory of plane partitions and may be used to compute the number of symmetric plane partitions as well as the number of shifted plane partitions in $B(n, n, 2 m)$ such that (in both cases) the parts along the main diagonal are even [76, 88].

Another notable special case follows when $q=0$. For $s \in \lambda \subset(2 m)^{n}$ such that $a^{\prime}(s)$ is even we must have $2 m-a^{\prime}(s) \geqslant 2$, which implies that $b_{\lambda ; m}^{\text {oa }}(0, t)=1$. By (2.59) the $q=0$ specialisation of (4.5) is thus

$$
\sum_{\substack{\lambda \text { even } \\ \lambda_{1} \leqslant 2 m}} P_{\lambda}(x ; t)=\left(x_{1} \cdots x_{n}\right)^{m} P_{m^{n}}^{\left(\mathrm{C}_{n}\right)}(x ; t, 0) .
$$

For positive $m$ the right-hand side can be expressed in terms of the function $\Phi(x ; t, 0,0)$ by Lemma 2.4 . The resulting $t$-analogue of the DésarménienProctor-Stembridge determinant is due to Stembridge [88, Theorem 1.2] who used it to give new proofs of the Rogers-Ramanujan identities. We will see in

\footnotetext{
${ }^{7}$ By (2.27), the same result may be obtained in the $a \rightarrow \infty$ limit.
} 
Section 5.2 that Stembridge's method can be extended so that identities such as (4.7) yield Rogers-Ramanujan identities for certain affine Lie algebras $X_{N}^{(r)}$ for arbitrary $N$.

For $m$ a nonnegative integer and $\lambda$ a partition let

$$
b_{\lambda ; m}^{\mathrm{ol}}(q, t):=\prod_{\substack{s \in \lambda \\ l^{\prime}(s) \text { odd }}} \frac{1-q^{m-a^{\prime}(s)} t^{l^{\prime}(s)-1}}{1-q^{m-a^{\prime}(s)-1} t^{l^{\prime}(s)}} \prod_{\substack{s \in \lambda \\ l(s) \text { odd }}} \frac{1-q^{a(s)} t^{l(s)}}{1-q^{a(s)+1} t^{l(s)-1}} .
$$

Note that $b_{\lambda ; m}^{\text {ol }}(q, t)=0$ if $\lambda_{2}>m$, which implies vanishing for $\lambda_{1}>m$ when $\lambda^{\prime}$ is even. Our next theorem contains the first of two bounded analogues of (4.2) for $a=0$.

Theorem 4.2. For $x=\left(x_{1}, \ldots, x_{n}\right)$ and $m$ a nonnegative integer,

$$
\sum b_{\lambda ; m}^{\mathrm{ol}}(q, t) P_{\lambda}(x ; q, t)=\left(x_{1} \cdots x_{n}\right)^{\frac{m}{2}} P_{\left(\frac{m}{2}\right)^{n}}^{\left(\mathrm{B}_{n}, \mathrm{~B}_{n}\right)}(x ; q, t, 1),
$$

where the sum is over partitions $\lambda \subset m^{n}$ such that $m_{i}(\lambda)$ is even for all $1 \leqslant$ $i \leqslant m-1$.

To see that (4.2) for $a=0$ follows in the large- $m$ limit we note that there are two types of partitions contributing to the sum on the left.

Type 1: Partitions $\lambda$ such that $m_{i}(\lambda)$ is even for all $1 \leqslant i \leqslant m$, i.e., $\lambda^{\prime}$ is even.

Type 2: Partitions $\lambda$ such that $m_{i}(\lambda)$ is odd for $i=m$ and even for $1 \leqslant i<m$, i.e., $\lambda^{\prime}$ is odd and $\lambda_{1}=m$.

Macdonald polynomials indexed by partitions of Type 2 have degree at least $m$, so that their contribution vanishes in the large $m$ limit. Hence we are left with a sum over partitions of Type 1, for which

$$
\prod_{\substack{s \in \lambda \\ l(s) \text { odd }}} \frac{1-q^{a(s)} t^{l(s)}}{1-q^{a(s)+1} t^{l(s)-1}}=\prod_{\substack{s \in \lambda \\ l(s) \text { even }}} \frac{1-q^{a(s)} t^{l(s)+1}}{1-q^{a(s)+1} t^{l(s)}}=b_{\lambda}^{\mathrm{el}}(q, t),
$$

resulting in the $a=0$ case of (4.2). In fact, (2.45) can be used to dissect (4.8), resulting in two bounded Littlewood identities for $\mathrm{D}_{n}$, the first of which is our second bounded analogue of (4.2) for $a=0$.

Theorem 4.3. For $x=\left(x_{1}, \ldots, x_{n}\right)$ and $m$ a nonnegative integer,

$$
\begin{aligned}
& \sum_{\lambda^{\prime} \text { even }} b_{\lambda ; m}^{\text {ol }}(q, t) P_{\lambda}(x ; q, t)=\left(x_{1} \cdots x_{n}\right)^{\frac{m}{2}} P_{\left(\frac{m}{2}\right)^{n}}^{\left(\mathrm{D}_{n}, \mathrm{D}_{n}\right)}(x ; q, t) \\
& \sum_{\substack{\lambda^{\prime} \text { odd } \\
\lambda_{1}=m}} b_{\lambda ; m}^{\mathrm{ol}}(q, t) P_{\lambda}(x ; q, t)=\left(x_{1} \cdots x_{n}\right)^{\frac{m}{2}} P_{\left(\frac{m}{2}\right)^{n}}^{\left(\mathrm{D}_{n}, \mathrm{D}_{n}\right)}(\bar{x} ; q, t) .
\end{aligned}
$$


Taking $q=0$ in (4.9a) yields

$$
\sum_{\substack{\lambda^{\prime} \text { even } \\ \lambda_{1} \leqslant m}} P_{\lambda}(x ; t) \prod_{i=1}^{m-1}\left(t ; t^{2}\right)_{m_{i}(\lambda) / 2}=\left(x_{1} \cdots x_{n}\right)^{\frac{m}{2}} P_{\left(\frac{m}{2}\right)^{n}}^{\left(\mathrm{D}_{n}\right)}(x ; t) .
$$

By Lemma 2.6 this is equivalent to [37, Theorem 1; Eq. (7)] of Jouhet and Zeng, which itself is a t-analogue of Okada's determinant [72, Theorem 2.3 $(3)]$

$$
\sum_{\substack{\lambda^{\prime} \text { even } \\ \lambda_{1} \leqslant m}} s_{\lambda}(x)=\frac{\sum_{\varepsilon \in\{ \pm 1\}} \operatorname{det}_{1 \leqslant i, j \leqslant n}\left(x_{i}^{j-1}+\varepsilon x_{i}^{m+2 n-j-1}\right)}{2 \prod_{i<j}\left(x_{i}-x_{j}\right)\left(x_{i} x_{j}-1\right)} .
$$

For $m$ a nonnegative integer and $\lambda$ a partition let

$$
b_{\lambda ; m}^{\mathrm{el}}(q, t):=b_{\lambda}^{\mathrm{el}}(q, t) \prod_{\substack{s \in \lambda \\ l^{\prime}(s) \text { even }}} \frac{1-q^{m-a^{\prime}(s)} t^{l^{\prime}(s)}}{1-q^{m-a^{\prime}(s)-1} t^{l^{\prime}(s)+1}} .
$$

We note that $b_{\lambda ; m}^{\mathrm{el}}(q, t)$ vanishes unless $\lambda_{1} \leqslant m$. The next result is (1.3) from the introduction, which bounds (4.2) for $a=1$.

Theorem 4.4. For $x=\left(x_{1}, \ldots, x_{n}\right)$ and $m$ a nonnegative integer,

$$
\sum_{\lambda} b_{\lambda ; m}^{\mathrm{el}}(q, t) P_{\lambda}(x ; q, t)=\left(x_{1} \cdots x_{n}\right)^{\frac{m}{2}} P_{\left(\frac{m}{2}\right)^{n}}^{\left(\mathrm{B}_{n}, \mathrm{~B}_{n}\right)}(x ; q, t, t) .
$$

The $q=0$ and $t=q$ specialisations of (4.11) correspond to (4.16) for $t_{2}=t$, i.e.,

$$
\sum_{\substack{\lambda \\ \lambda_{1} \leqslant m}} \prod_{i=1}^{m-1}\left(\left(t ; t^{2}\right)_{\left\lceil m_{i}(\lambda) / 2\right\rceil}\right) P_{\lambda}(x ; t)=\left(x_{1} \cdots x_{n}\right)^{\frac{m}{2}} P_{\left(\frac{m}{2}\right)^{n}}^{\left(\mathrm{B}_{n}\right)}(x ; t, t),
$$

and Macdonald's determinant (1.2) respectively.

Remark 4.5. Using (4.9a) it is not hard to prove an identity that generalises (4.2) in full:

$$
\sum_{\lambda} a^{\operatorname{odd}\left(\lambda^{\prime}\right)} \hat{b}_{\lambda ; m}^{\mathrm{el}}(q, t) P_{\lambda}(x ; q, t)=\left(\prod_{i=1}^{n} x_{i}^{m / 2} \frac{\left(a t x_{i} ; q\right)_{\infty}}{\left(a x_{i} ; q\right)_{\infty}}\right) P_{\left(\frac{m}{2}\right)^{n}}^{\left(\mathrm{D}_{n}, \mathrm{D}_{n}\right)}(x ; q, t),
$$

where

$$
\hat{b}_{\lambda ; m}^{\mathrm{el}}(q, t):=b_{\lambda}^{\mathrm{el}}(q, t) \prod_{\substack{s \in \lambda \\ l^{\prime}(s) \text { odd }}} \frac{1-q^{m-a^{\prime}(s)} t^{l^{\prime}(s)-1}}{1-q^{m-a^{\prime}(s)-1} t^{l^{\prime}(s)}} .
$$

The largest part of $\lambda$ in the sum on the left is not bounded, and unlike (4.8), (4.9a) or (4.11), this is not a polynomial identity. 
For $m$ a nonnegative integer and $\lambda$ a partition such that $\lambda_{1} \leqslant m$, let

$$
b_{\lambda ; m}^{-}(q, t):=b_{\lambda}^{-}(q, t) \prod_{s \in \lambda} \frac{1-q^{m-a^{\prime}(s)} t^{l^{\prime}(s)}}{1+q^{m-a^{\prime}(s)-1} t^{l^{\prime}(s)+1}} .
$$

Our final result for Macdonald polynomials is a bounded analogue of Kawanaka's conjecture (4.3).

Theorem 4.6. For $x=\left(x_{1}, \ldots, x_{n}\right)$ and $m$ a nonnegative integer,

$$
\sum_{\lambda} b_{\lambda ; m}^{-}(q, t) P_{\lambda}\left(x ; q^{2}, t^{2}\right)=\left(x_{1} \cdots x_{n}\right)^{\frac{m}{2}} P_{\left(\frac{m}{2}\right)^{n}}^{\left(\mathrm{B}_{n}, \mathrm{C}_{n}\right)}\left(x ; q^{2}, t^{2},-t\right) .
$$

For $t=-q$ this simplifies to (1.2) and for $q=0$ it is (4.16) below with $\left(t, t_{2}\right) \mapsto\left(t^{2},-t\right)$, viz.

$$
\sum_{\substack{\lambda \\ \lambda_{1} \leqslant m}} \prod_{i=1}^{m-1}\left((-t ; t)_{m_{i}(\lambda)}\right) P_{\lambda}\left(x ; t^{2}\right)=\left(x_{1} \cdots x_{n}\right)^{\frac{m}{2}} P_{\left(\frac{m}{2}\right)^{n}}^{\left(\mathrm{B}_{n}\right)}\left(x ; t^{2},-t\right) .
$$

Assuming $m$ is positive and rewriting the right-hand side using Lemma 2.5 yields [34, Theorem 1] of Ishikawa et al.

4.1.2. $t$-Identities. Our final two theorems do not appear to have simple analogues for Macdonald polynomials.

Recall the generalised Rogers-Szegö polynomials (2.8).

Theorem 4.7. For $x=\left(x_{1}, \ldots, x_{n}\right)$ and $m$ a nonnegative integer,

$$
\sum_{\substack{\lambda \\ \lambda_{1} \leqslant 2 m}} h_{\lambda}^{(2 m)}\left(t_{2}, t_{3} ; t\right) P_{\lambda}(x ; t)=\left(x_{1} \cdots x_{n}\right)^{m} P_{m^{n}}^{\left(\mathrm{BC}_{n}\right)}\left(x ; t, t_{2}, t_{3}\right) .
$$

This bounds [96, Theorem 1.1]

$$
\sum_{\lambda} h_{\lambda}\left(t_{2}, t_{3} ; t\right) P_{\lambda}(x ; t)=\prod_{i=1}^{n} \frac{\left(1-t_{2} x_{i}\right)\left(1-t_{3} x_{i}\right)}{1-x_{i}^{2}} \prod_{1 \leqslant i<j \leqslant n} \frac{1-t x_{i} x_{j}}{1-x_{i} x_{j}}
$$

with $h_{\lambda}\left(t_{2}, t_{3} ; t\right)$ given by (2.9). Moreover, if we replace $\left(t, t_{2}, t_{3}\right) \mapsto(0,-a,-b)$ and use (2.55) and $H_{m}(z ; 0)=1+z+\cdots+z^{m}$, we obtain the following twoparameter generalisation of the Désarménien-Proctor-Stembridge determinant (4.6):

$$
\begin{aligned}
& \sum_{\substack{\lambda \\
\lambda_{1} \leqslant 2 m}} s_{\lambda}(x) \prod_{\substack{i=1 \\
i \text { odd }}}^{2 m-1} \frac{a^{m_{i}(\lambda)+1}-b^{m_{i}(\lambda)+1}}{a-b} \prod_{\substack{i=1 \\
i \text { even }}}^{2 m-1} \frac{1-(a b)^{m_{i}(\lambda)+1}}{1-a b} \\
& =\frac{\operatorname{det}_{1 \leqslant i, j \leqslant n}\left(x_{i}^{j-1}\left(1+a x_{i}\right)\left(1+b x_{i}\right)-x_{i}^{2 m+2 n-j-1}\left(x_{i}+a\right)\left(x_{i}+b\right)\right)}{\prod_{i=1}^{n}\left(1-x_{i}^{2}\right) \prod_{1 \leqslant i<j \leqslant n}\left(x_{i}-x_{j}\right)\left(x_{i} x_{j}-1\right)} .
\end{aligned}
$$


Recall (2.10) . The $t_{3}=-1$ case of Theorem 4.7 extends as follows.

Theorem 4.8. For $x=\left(x_{1}, \ldots, x_{n}\right)$ and $m$ a nonnegative integer,

$$
\sum_{\substack{\lambda \\ \lambda_{1} \leqslant m}} h_{\lambda}^{(m)}\left(t_{2} ; t\right) P_{\lambda}(x ; t)=\left(x_{1} \cdots x_{n}\right)^{\frac{m}{2}} P_{\left(\frac{m}{2}\right)^{n}}^{\left(\mathrm{B}_{n}\right)}\left(x ; t, t_{2}\right) .
$$

This is stated without proof in [96]. For $\left(t, t_{2}\right) \mapsto(0,-a)$ it simplifies to a one-parameter generalisation of Macdonald's determinant (1.2) from the introduction:

$$
\sum_{\substack{\lambda \\ \lambda_{1} \leqslant m}} s_{\lambda}(x) \prod_{i=1}^{m-1} \frac{1-a^{m_{i}(\lambda)+1}}{1-a}=\frac{\operatorname{det}_{1 \leqslant i, j \leqslant n}\left(x_{i}^{j-1}\left(1+a x_{i}\right)-x_{i}^{m+2 n-j-1}\left(x_{i}+a\right)\right)}{\prod_{i=1}^{n}\left(1-x_{i}\right) \prod_{1 \leqslant i<j \leqslant n}\left(x_{i}-x_{j}\right)\left(x_{i} x_{j}-1\right)} .
$$

\subsection{Proofs of Theorems 4.1 4.8.}

We begin by outlining the general strategy, which is to transform the problem of proving bounded Littlewood identities into that of evaluating virtual Koornwinder integrals.

Recall that if $g \in \Lambda_{n}$ and $\left\{f_{\lambda}\right\}$ is a basis of $\Lambda_{n}$, then $\left[f_{\lambda}\right] g$ is the coefficient of $f_{\lambda}$ in the expansion of $g$. Working in full generality, we would like to find a closed-form expression for

$$
f_{\lambda}^{(m)}\left(q, t ; t_{0}, t_{1}, t_{2}, t_{3}\right):=\left[P_{\lambda}(x ; q, t)\right]\left(x_{1} \cdots x_{n}\right)^{m} K_{m^{n}}\left(x ; q, t ; t_{0}, t_{1}, t_{2}, t_{3}\right),
$$

where $m$ is a nonnegative integer. Since

$$
\left(x_{1} \cdots x_{n}\right)^{m} K_{m^{n}}\left(x ; q, t ; t_{0}, t_{1}, t_{2}, t_{3}\right)=\sum_{\lambda \subset(2 m)^{n}} u_{\lambda} m_{\lambda}(x),
$$

it follows that $f_{\lambda}^{(m)}\left(q, t ; t_{0}, t_{1}, t_{2}, t_{3}\right)$ vanishes unless $\lambda \subset(2 m)^{n}$.

Proposition 4.9. For $m$ a nonnegative integer and $\lambda \subset(2 m)^{n}$,

$$
f_{\lambda}^{(m)}\left(q, t ; t_{0}, t_{1}, t_{2}, t_{3}\right)=(-1)^{|\lambda|} I_{K}^{(m)}\left(P_{\lambda^{\prime}}(t, q) ; t, q ; t_{0}, t_{1}, t_{2}, t_{3}\right) .
$$

Proof. Let $x=\left(x_{1}, \ldots, x_{n}\right)$ and $y=\left(y_{1}, \ldots, y_{m}\right)$. According to the Cauchy identity for Koornwinder polynomials (2.34)

$$
\begin{array}{r}
\sum_{\lambda \subset m^{n}}(-1)^{|\lambda|}\left(x_{1} \cdots x_{n}\right)^{m} K_{m^{n}-\lambda}\left(x ; q, t ; t_{0}, t_{1}, t_{2}, t_{3}\right) K_{\lambda^{\prime}}\left(y ; t, q ; t_{0}, t_{1}, t_{2}, t_{3}\right) \\
=\sigma_{1}\left[-x y^{ \pm}\right] .
\end{array}
$$


If we expand the right-hand side in terms of Macdonald polynomials using the Cauchy identity (2.20) this yields

$$
\begin{aligned}
\sum_{\lambda \subset m^{n}}(-1)^{|\lambda|}\left(x_{1} \cdots x_{n}\right)^{m} K_{m^{n}-\lambda}(x ; q, t & \left.; t_{0}, t_{1}, t_{2}, t_{3}\right) K_{\lambda^{\prime}}\left(y ; t, q ; t_{0}, t_{1}, t_{2}, t_{3}\right) \\
& =\sum_{\lambda \subset(2 m)^{n}}(-1)^{|\lambda|} P_{\lambda}(x ; q, t) P_{\lambda^{\prime}}\left(y^{ \pm} ; t, q\right) .
\end{aligned}
$$

Equating coefficients of $P_{\lambda}(x ; q, t) K_{0}\left(y ; t, q ; t_{0}, t_{1}, t_{2}, t_{3}\right)$ we find

$$
\begin{aligned}
& {\left[P_{\lambda}(x ; q, t)\right]\left(x_{1} \cdots x_{n}\right)^{m} K_{m^{n}}\left(x ; q, t ; t_{0}, t_{1}, t_{2}, t_{3}\right)} \\
& =(-1)^{|\lambda|}\left[K_{0}\left(y ; t, q ; t_{0}, t_{1}, t_{2}, t_{3}\right)\right] P_{\lambda^{\prime}}\left(y^{ \pm} ; t, q\right),
\end{aligned}
$$

for $\lambda \subset(2 m)^{n}$. Recalling (3.2) and (4.17) completes the proof.

Next we consider the problem of computing

$$
f_{\lambda}^{(m)}\left(q, t ; t_{2}, t_{3}\right):=\left[P_{\lambda}(x ; q, t)\right]\left(x_{1} \cdots x_{n}\right)^{m} K_{m^{n}}\left(x ; q, t ; t_{2}, t_{3}\right),
$$

where $m$ is a nonnegative integer or half-integer and $K_{\lambda}\left(x ; q, t ; t_{2}, t_{3}\right)$ is the Macdonald-Koornwinder polynomial of Section 2.7.2.

Proposition 4.10. For $m$ a nonnegative integer or half-integer, $\lambda \subset(2 m)^{n}$ and generic $q, t, t_{2}, t_{3}$

$$
f_{\lambda}^{(m)}\left(q, t ; t_{2}, t_{3}\right)=(-1)^{|\lambda|} I_{K}\left(P_{\lambda^{\prime}}(t, q) ; t, q, q^{m} ;-1,-q^{1 / 2}, t_{2}, t_{3}\right) .
$$

Proof. When $m$ is an integer we simply have

$$
f_{\lambda}^{(m)}\left(q, t ; t_{2}, t_{3}\right)=f_{\lambda}^{(m)}\left(q, t ;-1,-q^{1 / 2}, t_{2}, t_{3}\right) .
$$

By (4.18) this gives

$$
f_{\lambda}^{(m)}\left(q, t ; t_{2}, t_{3}\right)=(-1)^{|\lambda|} I_{K}^{(m)}\left(P_{\lambda^{\prime}}(t, q) ; t, q ;-1,-q^{1 / 2}, t_{2}, t_{3}\right),
$$

which may also be written as (4.21).

To deal with the half-integer case we set $k=m-1 / 2$ and replace $m$ by $k$ in (4.19), so that now $y=\left(y_{1}, \ldots, y_{k}\right)$. Multiplying both sides by $\prod_{i=1}^{n}\left(1+x_{i}\right)=$ $\sigma_{1}[-\varepsilon x]$, using that

$$
\sigma_{1}[-\varepsilon x] \sigma_{1}\left[-x y^{ \pm}\right]=\sigma_{1}\left[-\varepsilon x-x y^{ \pm}\right]=\sigma_{1}\left[-x\left(y^{ \pm}+\varepsilon\right)\right],
$$


and then expanding this using the Cauchy identity (2.20) we obtain

$$
\begin{aligned}
& \prod_{i=1}^{n}\left(1+x_{i}\right) \\
& \quad \times \sum_{\lambda \subset k^{n}}(-1)^{|\lambda|}\left(x_{1} \cdots x_{n}\right)^{k} K_{k^{n}-\lambda}\left(x ; q, t ; t_{0}, t_{1}, t_{2}, t_{3}\right) K_{\lambda^{\prime}}\left(y ; t, q ; t_{0}, t_{1}, t_{2}, t_{3}\right) \\
& =\sum_{\lambda \subset(2 m)^{n}}(-1)^{|\lambda|} P_{\lambda}(x ; q, t) P_{\lambda^{\prime}}\left(\left[y^{ \pm}+\varepsilon\right] ; t, q\right) .
\end{aligned}
$$

After specialising $\left\{t_{0}, t_{1}\right\}=\left\{-q,-q^{1 / 2}\right\}$ we can apply Lemma 2.3 to rewrite this as

$$
\begin{aligned}
\sum_{\lambda \subset k^{n}}(-1)^{|\lambda|}\left(x_{1} \cdots x_{n}\right)^{m} K_{m^{n}-\lambda}\left(x ; q, t ; t_{2}, t_{3}\right) K_{\lambda^{\prime}}\left(y ; t, q ;-q,-q^{1 / 2}, t_{2}, t_{3}\right) \\
=\sum_{\lambda \subset(2 m)^{n}}(-1)^{|\lambda|} P_{\lambda}(x ; q, t) P_{\lambda^{\prime}}\left(\left[y^{ \pm}+\varepsilon\right] ; t, q\right) .
\end{aligned}
$$

Equating coefficients of $P_{\lambda}(x ; q, t) K_{0}\left(y ; t, q ;-q,-q^{1 / 2}, t_{2}, t_{3}\right)$ yields

$$
f_{\lambda}^{(m)}\left(q, t ; t_{2}, t_{3}\right)=(-1)^{|\lambda|} I_{K}^{(k)}\left(P_{\lambda^{\prime}}([y+\varepsilon] ; t, q) ; t, q ;-q,-q^{1 / 2}, t_{2}, t_{3}\right)
$$

for $\lambda \subset(2 m)^{n}$. For generic $q, t, t_{2}, t_{3}$ we can write the integral on the right as

$$
I_{K}\left(P_{\lambda^{\prime}}\left(\left[y^{ \pm}+\varepsilon\right] ; t, q\right) ; t, q, q^{k} ;-q,-q^{1 / 2}, t_{2}, t_{3}\right),
$$

where now $y=\left(y_{1}, y_{2}, \ldots\right)$. By Lemma 3.3 this is also

$$
I_{K}\left(P_{\lambda^{\prime}}(t, q) ; t, q, q^{m} ;-1,-q^{1 / 2}, t_{2}, t_{3}\right) .
$$

We are now ready prove Theorems 4.14.8,

Proof of Theorem 4.1. We first prove the $a=0$ case, given in (4.5). By definition (4.17) and equation (2.47) this is equivalent to proving that for $\lambda \subset(2 m)^{n}$

$$
f_{\lambda}^{(m)}\left(q, t ; \pm q^{1 / 2}, \pm(q t)^{1 / 2}\right)= \begin{cases}b_{\lambda ; m}^{\text {oa }}(q, t) & \lambda \text { is even } \\ 0 & \text { otherwise. }\end{cases}
$$

From (4.18) we have

$$
f_{\lambda}^{(m)}\left(q, t ; \pm q^{1 / 2}, \pm(q t)^{1 / 2}\right)=(-1)^{|\lambda|} I_{K}^{(m)}\left(P_{\lambda^{\prime}}(t, q) ; t, q ; \pm q^{1 / 2}, \pm(q t)^{1 / 2}\right) .
$$

Taking $(T, \mu)=\left(t^{m}, \lambda^{\prime}\right)$ in (3.8) and then interchanging $q$ and $t$ it follows that the virtual Koornwinder integral on the right vanishes unless $\lambda$ is even in which case it evaluates in closed form to

$$
\frac{\left(q^{2 m} ; t, q^{2}\right)_{(\lambda / 2)^{\prime}}}{\left(q^{2 m-1} t ; t, q^{2}\right)_{(\lambda / 2)^{\prime}}} \cdot \frac{C_{(\lambda / 2)^{\prime}}^{-}\left(q t ; t, q^{2}\right)}{C_{(\lambda / 2)^{\prime}}^{-}\left(q^{2} ; t, q^{2}\right)} .
$$


Using (2.4) we thus find

$$
f_{\lambda}^{(m)}\left(q, t ; \pm q^{1 / 2}, \pm(q t)^{1 / 2}\right)=\left(\frac{q}{t}\right)^{|\lambda| / 2} \frac{\left(q^{-2 m} ; q^{2}, t\right)_{\lambda / 2}}{\left(q^{1-2 m} / t ; q^{2}, t\right)_{\lambda / 2}} \cdot \frac{C_{\lambda / 2}^{-}\left(q t ; q^{2}, t\right)}{C_{\lambda / 2}^{-}\left(q^{2} ; q^{2}, t\right)}
$$

for $\lambda$ even and zero otherwise. By (2.2) the right can be also be written as

$$
\begin{aligned}
f_{\lambda}^{(m)}\left(q, t ; \pm q^{1 / 2}, \pm(q t)^{1 / 2}\right) & \\
= & \prod_{s \in \eta}\left(\frac{1-q^{2 m-2 a^{\prime}(s)} t^{l^{\prime}(s)}}{1-q^{2 m-2 a^{\prime}(s)-1} t^{l^{\prime}(s)+1}} \cdot \frac{1-q^{2 a(s)+1} t^{l(s)+1}}{1-q^{2 a(s)+2} t^{l(s)}}\right) \\
& =\prod_{\substack{s \in \lambda \\
a(s) \text { odd }}}\left(\frac{1-q^{2 m-a^{\prime}(s)} t^{l^{\prime}(s)}}{1-q^{2 m-a^{\prime}(s)-1} t^{l^{(s)+1}}} \cdot \frac{1-q^{a(s)} t^{l(s)+1}}{1-q^{a(s)+1} t^{l(s)}}\right) .
\end{aligned}
$$

Since $\lambda$ is even, odd arms-lengths correspond to even arm-colengths. The product on the right is thus $b_{\lambda ; m}^{\text {oa }}(q, t)$ in the representation given by (4.4), completing the proof of (4.5).

To obtain the full theorem we multiply both sides of (4.5) by $\prod_{i=1}^{n}\left(1+a x_{i}\right)$. By the $e$-Pieri rule (2.23) we must then show that

$$
b_{\lambda ; m}^{\mathrm{oa}}(q, t)=\sum_{\mu \text { even }} a^{|\lambda / \mu|} \psi_{\lambda / \mu}^{\prime}(q, t) b_{\mu ; m}^{\mathrm{oa}}(q, t) .
$$

Because $\mu$ is even and $\psi_{\lambda / \mu}^{\prime}(q, t)$ vanishes unless $\lambda / \mu$ is a vertical strip, $\mu$ is fixed as

$$
\mu=2\lfloor\lambda / 2\rfloor:=\left(2\left\lfloor\lambda_{1} / 2\right\rfloor, 2\left\lfloor\lambda_{2} / 2\right\rfloor, \ldots\right),
$$

which implies that $|\lambda / \mu|=\operatorname{odd}(\lambda)$. We thus obtain

$$
b_{\lambda ; m}^{\mathrm{oa}}(q, t)=\psi_{\lambda / \mu}^{\prime}(q, t) b_{\mu ; m}^{\mathrm{oa}}(q, t)
$$

with $\mu$ fixed as above. The $m$-dependent parts on both sides trivially agree since

$$
\prod_{\substack{s \in \lambda \\ a^{\prime}(s) \text { odd }}} f_{a^{\prime}(s), l^{\prime}(s)}=\prod_{\substack{s \in \mu \\ a^{\prime}(s) \text { odd }}} f_{a^{\prime}(s), l^{\prime}(s)}
$$

It thus remains to show that

$$
b_{\lambda}^{\mathrm{oa}}(q, t)=\psi_{\lambda / \mu}^{\prime}(q, t) b_{\mu}^{\mathrm{oa}}(q, t) .
$$

Replacing $(\lambda, \mu, q, t)$ by $\left(\lambda^{\prime}, \mu^{\prime}, t, q\right)$, using

$$
b_{\nu^{\prime}}^{\mathrm{oa}}(t, q)=\frac{b_{\nu}^{\mathrm{el}}(q, t)}{b_{\nu}(q, t)}
$$


on either side, and finally appealing to [64, page 341]

$$
\psi_{\lambda^{\prime} / \mu^{\prime}}^{\prime}(t, q)=\varphi_{\lambda / \mu}(q, t) \frac{b_{\mu}(q, t)}{b_{\lambda}(q, t)}
$$

we are left with

$$
b_{\lambda}^{\mathrm{el}}(q, t)=\varphi_{\lambda / \mu}(q, t) b_{\mu}^{\mathrm{el}}(q, t)
$$

for $\mu^{\prime}=2\left\lfloor\lambda^{\prime} / 2\right\rfloor$. This is [64, p. 351] so that we are done.

Because they are simpler to prove than Theorems 4.2 and 4.3 we consider Theorems 4.4 and 4.6 first.

Proof of Theorem 4.4. It will be convenient to prove the claim with $m$ replaced by $2 m$. After this change $m$ is a nonnegative integer or half-integer. It then follows from (4.20) and (2.50a) that we must prove for $\lambda \subset(2 m)^{n}$ that

$$
f_{\lambda}^{(m)}\left(q, t ; t, q^{1 / 2}\right)=b_{\lambda ; 2 m}^{\mathrm{el}}(q, t) .
$$

By Proposition 4.10

$$
f_{\lambda}^{(m)}\left(q, t ; t, q^{1 / 2}\right)=(-1)^{|\lambda|} I_{K}\left(P_{\lambda^{\prime}}(t, q) ; t, q, q^{m} ;-1, t, \pm q^{1 / 2}\right) .
$$

The integral on the right can be computed by Theorem 3.6 with $(q, t, T, \mu) \mapsto$ $\left(t, q, q^{m}, \lambda^{\prime}\right)$, resulting in

$$
f_{\lambda}^{(m)}\left(q, t ; t, q^{1 / 2}\right)=\frac{\left(q^{2 m} ; t^{2}, q\right)_{\left\lceil\lambda^{\prime} / 2\right\rceil}}{\left(q^{2 m-1} t ; t^{2}, q\right)_{\left\lceil\lambda^{\prime} / 2\right\rceil}} \cdot \frac{1}{b_{\lambda^{\prime}}^{\text {ea }}(t, q)} .
$$

Let $\nu:=\left\lceil\lambda^{\prime} / 2\right\rceil^{\prime}=\left(\lambda_{1}, \lambda_{3}, \ldots\right)$. By (2.4a) we can write the first factor on the right as

$$
\left(\frac{q}{t}\right)^{|\nu|} \frac{\left(q^{-2 m} ; q, t^{2}\right)_{\nu}}{\left(q^{1-2 m} / t ; q, t^{2}\right)_{\nu}}
$$

By (2.2) this is also

$$
\prod_{s \in \nu} \frac{1-q^{2 m-a^{\prime}(s)} t^{2 l^{\prime}(s)}}{1-q^{2 m-a^{\prime}(s)-1} t^{2 l^{\prime}(s)+1}}=\prod_{\substack{s \in \lambda \\ l^{\prime}(s) \text { even }}} \frac{1-q^{2 m-a^{\prime}(s)} t^{l^{\prime}(s)}}{1-q^{2 m-a^{\prime}(s)-1} t^{l^{\prime}(s)+1}} .
$$

Since under conjugation legs become arms and arms become legs, we further have

$$
b_{\lambda^{\prime}}^{\mathrm{ea}}(t, q) b_{\lambda}^{\mathrm{el}}(q, t)=1
$$

Hence

$$
f_{\lambda}^{(m)}\left(q, t ; t, q^{1 / 2}\right)=b_{\lambda}^{\mathrm{el}}(q, t) \prod_{\substack{s \in \lambda \\ l^{\prime}(s) \text { even }}} \frac{1-q^{2 m-a^{\prime}(s)} t^{l^{\prime}(s)}}{1-q^{2 m-a^{\prime}(s)-1} t^{l^{\prime}(s)+1}}=b_{\lambda ; 2 m}^{\mathrm{el}}(q, t)
$$

as claimed. 
Proof of Theorem 4.6. We closely follow the previous proof and again replace $m$ by $2 m$. This time it follows from (4.20) and (2.50b) that we must prove

$$
f_{\lambda}^{(m)}\left(q^{2}, t^{2} ;-t,-q t\right)=b_{\lambda ; 2 m}^{-}(q, t)
$$

for $\lambda \subset(2 m)^{n}$. By Proposition 4.10

$$
f_{\lambda}^{(m)}\left(q^{2}, t^{2} ;-t,-q t\right)=(-1)^{|\lambda|} I_{K}\left(P_{\lambda^{\prime}}\left(t^{2}, q^{2}\right) ; t^{2}, q^{2}, q^{2 m} ;-1,-q,-t,-q t\right) .
$$

The integral on the right evaluates to

$$
(-1)^{|\lambda|} \frac{\left(q^{2 m} ; t, q\right)_{\lambda^{\prime}}}{\left(-q^{2 m-1} t ; t, q\right)_{\lambda^{\prime}}} \cdot \frac{C_{\lambda^{\prime}}^{-}(-t ; t, q)}{C_{\lambda^{\prime}}^{-}(q ; t, q)} .
$$

by (3.9) with $(q, t, T, \mu) \mapsto\left(t^{2}, q^{2}, t^{2 m}, \lambda^{\prime}\right)$. Also using (2.4) we find

$$
f_{\lambda}^{(m)}\left(q^{2}, t^{2} ;-t,-q t\right)=\left(-\frac{q}{t}\right)^{|\lambda|} \frac{\left(q^{-2 m} ; q, t\right)_{\lambda}}{\left(-q^{1-2 m} / t ; q, t\right)_{\lambda}} \cdot \frac{C_{\lambda}^{-}(-t ; q, t)}{C_{\lambda}^{-}(q ; q, t)} .
$$

Equation (4.24) now follows by (2.2).

Proof of Theorem 4.2. Again we prove the theorem with $m$ replaced by $2 m$. It then follows from (4.20) and (2.50a) that we must prove for $\lambda \subset(2 m)^{n}$ that $f_{\lambda}^{(m)}\left(q, t ; 1, q^{1 / 2}\right)$ vanishes unless $m_{i}(\lambda)$ is even for all $1 \leqslant i \leqslant 2 m-1$, in which case

$$
f_{\lambda}^{(m)}\left(q, t ; 1, q^{1 / 2}\right)=b_{\lambda ; 2 m}^{\mathrm{ol}}(q, t) .
$$

The problem with using Proposition 4.10 as in the proof of Theorem 4.6 is that the specialisation $\left\{t_{2}, t_{3}\right\}=\left\{1, q^{1 / 2}\right\}$ corresponds to one of the non-generic cases discussed on page 28. It would lead to

$$
f_{\lambda}^{(m)}\left(q, t ; 1, q^{1 / 2}\right)=(-1)^{|\lambda|} I_{K}\left(P_{\lambda^{\prime}}(t, q) ; t, q, q^{m} ; \pm 1, \pm q^{1 / 2}\right),
$$

where the integral on the right is not well-defined. It is still possible to use (4.26) by interpreting the right in an appropriate limiting sense, but instead we proceed slightly differently.

First, when $m$ is an integer (4.21) simply says that

$$
f_{\lambda}^{(m)}\left(q, t ; t_{2}, t_{3}\right)=(-1)^{|\lambda|} I_{K}^{(m)}\left(P_{\lambda^{\prime}}(t, q) ; t, q ;-1,-q^{1 / 2}, t_{2}, t_{3}\right) .
$$

In this equation there is no problem specialising $\left\{t_{2}, t_{3}\right\}=\left\{1, q^{1 / 2}\right\}$ so that

$$
f_{\lambda}^{(m)}\left(q, t ; 1, q^{1 / 2}\right)=(-1)^{|\lambda|} I_{K}^{(m)}\left(P_{\lambda^{\prime}}(t, q) ; t, q ; \pm 1, \pm q^{1 / 2}\right) .
$$

The right-hand side can be computed by Theorem 3.4 with $(\mu, q, t, n) \mapsto$ $\left(\lambda^{\prime}, t, q, m\right)$ so that

$$
f_{\lambda}^{(m)}\left(q, t ; 1, q^{1 / 2}\right)= \begin{cases}A_{\lambda^{\prime} / 2}^{(2 m)}(t, q) & \text { if } \widetilde{\lambda^{\prime}} \text { is even } \\ 0 & \text { otherwise }\end{cases}
$$


where we have also used that $|\lambda|$ is even if $\widetilde{\lambda^{\prime}}=\left(\lambda_{1}^{\prime}-\lambda_{2 m}^{\prime}, \ldots, \lambda_{2 m-1}^{\prime}-\lambda_{2 m}^{\prime}, 0\right)$ is even.

When $m=k+1 / 2$ is a half-integer, we use (4.22) written as

$$
f_{\lambda}^{(m)}\left(q, t ; t_{2}, t_{3}\right)=(-1)^{|\lambda|} I_{K}^{(k)}\left(P_{\lambda^{\prime}}\left(x_{1}, \ldots, x_{k},-1 ; t, q\right) ; t, q ;-q,-q^{1 / 2}, t_{2}, t_{3}\right)
$$

instead of (4.21). Specialising $\left\{t_{2}, t_{3}\right\}=\left\{1, q^{1 / 2}\right\}$ this gives

$$
f_{\lambda}^{(m)}\left(q, t ; 1, q^{1 / 2}\right)=(-1)^{|\lambda|} I_{K}^{(k)}\left(P_{\lambda^{\prime}}\left(x_{1}, \ldots, x_{k},-1 ; t, q\right) ; t, q ; 1,-q, \pm q^{1 / 2}\right) .
$$

Now the right can be computed by Theorem 3.5 with $(\nu, q, t, n) \mapsto\left(\lambda^{\prime}, t, q, k\right)$. Since $2 k+1=2 m$ and $|\lambda|+\lambda_{2 m}$ is even if $\widetilde{\lambda^{\prime}}$ is even, this once again results in (4.27).

To complete the proof we first note that $A_{\lambda^{\prime} / 2}^{(2 m)}(t, q)$ can be written as $A_{\left\lfloor\lambda^{\prime} / 2\right\rfloor}^{(2 m)}(t, q)$. Indeed, either $\lambda^{\prime}$ is even, in which case $\left\lfloor\lambda^{\prime} / 2\right\rfloor=\lambda^{\prime} / 2$ or $\lambda^{\prime}$ is odd and $\lambda_{1}=2 m$, in which case $\left\lfloor\lambda^{\prime} / 2\right\rfloor=\lambda^{\prime} / 2-(1 / 2)^{2 m}$. Since $A_{\lambda^{\prime} / 2}^{(2 m)}(t, q)$ only depends on the relative differences between the parts of $\lambda^{\prime} / 2$ the change is justified. Denoting $\left\lfloor\lambda^{\prime} / 2\right\rfloor$ by $\nu^{\prime}$ we find that in the non-vanishing case, that is, when $m_{i}(\lambda)$ is even for all $1 \leqslant i \leqslant 2 m-1$,

$$
\begin{aligned}
f_{\lambda}^{(m)}\left(q, t ; 1, q^{1 / 2}\right) & =A_{\nu^{\prime}}^{(2 m)}(t, q) \\
& =\frac{\left(q^{2 m} ; t^{2}, q\right)_{\nu^{\prime}}}{\left(q^{2 m-1} t ; t^{2}, q\right)_{\nu^{\prime}}} \cdot \frac{C_{\nu^{\prime}}^{-}\left(t ; t^{2}, q\right)}{C_{\nu^{\prime}}^{-}\left(q ; t^{2}, q\right)} \\
& =\left(\frac{q}{t}\right)^{|\nu|} \frac{\left(q^{-2 m} ; q, t^{2}\right)_{\nu}}{\left(q^{1-2 m} / t ; q, t^{2}\right)_{\nu}} \cdot \frac{C_{\nu}^{-}\left(t ; q, t^{2}\right)}{C_{\nu}^{-}\left(q ; q, t^{2}\right)}
\end{aligned}
$$

where the second equality follows from (3.12) and the last equality from (2.4). Since $\nu^{\prime}=\left\lfloor\lambda^{\prime} / 2\right\rfloor$ we also have $\nu=\left\lfloor\lambda^{\prime} / 2\right\rfloor^{\prime}$ which can be simplified to $\nu=$ $\left(\lambda_{2}, \lambda_{4}, \lambda_{6}, \ldots\right)$. Recalling (2.2), we obtain

$$
f_{\lambda}^{(m)}\left(q, t ; 1, q^{1 / 2}\right)=\prod_{s \in \nu}\left(\frac{1-q^{2 m-a^{\prime}(s)} t^{2 l^{\prime}(s)}}{1-q^{2 m-a^{\prime}(s)-1} t^{2 l^{\prime}(s)+1}} \cdot \frac{1-q^{a(s)} t^{2 l(s)+1}}{1-q^{a(s)+1} t^{2 l(s)}}\right) .
$$

To write this without reference to the partition $\nu$ we consider both factors in the product separately. The first factor is trivial:

$$
\prod_{s \in \nu} \frac{1-q^{2 m-a^{\prime}(s)} t^{2 l^{\prime}(s)}}{1-q^{2 m-a^{\prime}(s)-1} t^{2 l^{\prime}(s)+1}}=\prod_{\substack{s \in \lambda \\ l^{\prime}(s) \text { odd }}} \frac{1-q^{2 m-a^{\prime}(s)} t^{l^{\prime}(s)-1}}{1-q^{2 m-a^{\prime}(s)-1} t^{l^{\prime}(s)}} .
$$

For the second factor we use that for $\lambda^{\prime}$ even we must have $\lambda_{2 i}=\lambda_{2 i-1}$ for all $i$. We can therefore redefine $\nu$ as

$$
\nu:= \begin{cases}\left(\lambda_{1}, \lambda_{3}, \ldots\right) & \text { if } \lambda^{\prime} \text { is even } \\ \left(\lambda_{2}, \lambda_{4}, \ldots\right) & \text { if } \lambda^{\prime} \text { is odd }\end{cases}
$$


But for such $\nu$

$$
\prod_{s \in \nu} \frac{1-q^{a(s)} t^{2 l(s)+1}}{1-q^{a(s)+1} t^{2 l(s)}}=\prod_{s \in \lambda} \frac{1-q^{a(s)} t^{l(s)}}{1-q^{a(s)+1} t^{l(s)}}
$$

in both cases. Combining (4.29) and (4.30) we obtain (4.25).

Proof of Theorem 4.3. When $m$ is odd the result is completely elementary. By (2.45b) and (2.46) we can write the right-hand side of (4.8) as

$$
\left(x_{1} \cdots x_{n}\right)^{\frac{m}{2}} P_{\left(\frac{m}{2}\right)^{n}}^{\left(\mathrm{D}_{n}, \mathrm{D}_{n}\right)}(x ; q, t)+\left(x_{1} \cdots x_{n}\right)^{\frac{m}{2}} P_{\left(\frac{m}{2}\right)^{n}}^{\left(\mathrm{D}_{n}, \mathrm{D}_{n}\right)}(\bar{x} ; q, t) .
$$

When $m$ is odd the first term is a polynomial of even degree whereas the second term is a polynomial of odd degree. Since partitions $\lambda$ of Type 1 have even size and partitions of Type 2 have size congruent to $m$ modulo 2 , it follows that for odd $m$ we may dissect (4.8) as in Corollary 4.3.

To prove the theorem for even $m$ we closely follow the proof of Proposition 4.10. In (4.19) we replace $m$ by $m-1=: k$ (we do not at this point assume that $m$ is even) and multiply both sides by $\prod_{i=1}^{n}\left(1-x_{i}^{2}\right)=\sigma_{1}[-x(1+\varepsilon)]$. Then expanding the right-hand side in terms of Macdonald polynomials using (2.20) gives

$$
\begin{aligned}
& \prod_{i=1}^{n}\left(1-x_{i}^{2}\right) \\
& \quad \times \sum_{\lambda \subset k^{n}}(-1)^{|\lambda|}\left(x_{1} \cdots x_{n}\right)^{k} K_{k^{n}-\lambda}\left(x ; q, t ; t_{0}, t_{1}, t_{2}, t_{3}\right) K_{\lambda^{\prime}}\left(y ; t, q ; t_{0}, t_{1}, t_{2}, t_{3}\right) \\
& =\sum_{\lambda \subset(2 m)^{n}}(-1)^{|\lambda|} P_{\lambda}(x ; q, t) P_{\lambda^{\prime}}\left(\left[y^{ \pm}+1+\varepsilon\right] ; t, q\right),
\end{aligned}
$$

where $y=\left(y_{1}, \ldots, y_{k}\right)$. If we specialise $\left\{t_{0}, t_{1}, t_{2}, t_{3}\right\}=\left\{ \pm q, \pm q^{1 / 2}\right\}$ and apply Lemma 2.3 followed by (2.50b) this leads to

$$
\begin{aligned}
& \prod_{i=1}^{n}\left(x_{i}^{-1 / 2}-x_{i}^{1 / 2}\right) \\
& \times \sum_{\lambda \subset k^{n}}(-1)^{|\lambda|}\left(x_{1} \cdots x_{n}\right)^{m} P_{\left(m-\frac{1}{2}\right)^{n}-\lambda}^{\left(\mathrm{B}_{n}, \mathrm{C}_{n}\right)}\left(x ; q, t, q^{1 / 2}\right) K_{\lambda^{\prime}}\left(y ; t, q ; \pm q, \pm q^{1 / 2}\right) \\
& \quad=\sum_{\lambda \subset(2 m)^{n}}(-1)^{|\lambda|} P_{\lambda}(x ; q, t) P_{\lambda^{\prime}}\left(\left[y^{ \pm}+1+\varepsilon\right] ; t, q\right) .
\end{aligned}
$$


Equating coefficients of $P_{\lambda}(x ; q, t) K_{0}\left(y ; t, q ; \pm q, \pm q^{1 / 2}\right)$ and then replacing $y$ by $x$ on the right yields

$$
\begin{aligned}
& {\left[P_{\lambda}(x ; q, t)\right]\left(x_{1} \cdots x_{n}\right)^{m} P_{\left(m-\frac{1}{2}\right)^{n}}^{\left(\mathrm{B}_{n}, \mathrm{C}_{n}\right)}\left(x ; q, t, q^{1 / 2}\right) \prod_{i=1}^{n}\left(x_{i}^{-1 / 2}-x_{i}^{1 / 2}\right)} \\
& =(-1)^{|\lambda|} I_{K}^{(m-1)}\left(P_{\lambda^{\prime}}\left(x_{1}^{ \pm}, \ldots, x_{m-1}^{ \pm}, \pm 1 ; t, q\right) ; t, q ; \pm q, \pm q^{1 / 2}\right) .
\end{aligned}
$$

By the integer- $m$ case of Proposition 4.10

$$
\begin{aligned}
& {\left[P_{\lambda}(x ; q, t)\right]\left(x_{1} \cdots x_{n}\right)^{m} K_{m^{n}}\left(x ; q, t ; t_{2}, t_{3}\right)} \\
& \quad=(-1)^{|\lambda|} I_{K}^{(m)}\left(P_{\lambda^{\prime}}(t, q) ; t, q ;-1,-q^{1 / 2}, t_{2}, t_{3}\right) .
\end{aligned}
$$

For $\left\{t_{2}, t_{3}\right\}=\left\{1, q^{1 / 2}\right\}$ this can also be written as

$$
\begin{aligned}
& {\left[P_{\lambda}(x ; q, t)\right]\left(x_{1} \cdots x_{n}\right)^{m} P_{m^{n}}^{\left(\mathrm{B}_{n}, \mathrm{~B}_{n}\right)}(x ; q, t, 1)} \\
& =(-1)^{|\lambda|} I_{K}^{(m)}\left(P_{\lambda^{\prime}}(t, q) ; t, q ; \pm 1, \pm q^{1 / 2}\right)
\end{aligned}
$$

thanks to (2.50a). Taking half the sum of (4.31) and (4.32) and recalling (2.45) it follows that

$$
\begin{aligned}
& {\left[P_{\lambda}(x ; q, t)\right]\left(x_{1} \cdots x_{n}\right)^{m} P_{m^{n}}^{\left(\mathrm{D}_{n}, \mathrm{D}_{n}\right)}(x ; q, t)} \\
& \quad=\frac{1}{2}(-1)^{|\lambda|} I_{K}^{(m)}\left(P_{\lambda^{\prime}}\left(x_{1}^{ \pm}, \ldots, x_{m}^{ \pm} ; t, q\right) ; t, q ; \pm 1, \pm q^{1 / 2}\right) \\
& \quad+\frac{1}{2}(-1)^{|\lambda|} I_{K}^{(m-1)}\left(P_{\lambda^{\prime}}\left(x_{1}^{ \pm}, \ldots, x_{m-1}^{ \pm}, \pm 1 ; t, q\right) ; t, q ; \pm q, \pm q^{1 / 2}\right) .
\end{aligned}
$$

Both virtual Koornwinder integrals on the right can be computed by Theorem 3.4. Since

$$
\chi\left({\widetilde{\lambda^{\prime}}}^{\text {even }}\right)\left(\frac{1}{2}+\frac{1}{2}(-1)^{\lambda_{2 m}^{\prime}}\right)=\chi\left(\lambda^{\prime} \text { even }\right)
$$

for $\tilde{\lambda}^{\prime}=\left(\lambda_{1}^{\prime}-\lambda_{2 m}^{\prime}, \ldots, \lambda_{2 m-1}^{\prime}-\lambda_{2 m}^{\prime}, 0\right)$, we find

$$
\left[P_{\lambda}(x ; q, t)\right]\left(x_{1} \cdots x_{n}\right)^{m} P_{m^{n}}^{\left(\mathrm{D}_{n}, \mathrm{D}_{n}\right)}(x ; q, t)=\chi\left(\lambda^{\prime} \text { even }\right) A_{\lambda^{\prime} / 2}(t, q) .
$$

In the proof of Theorem 4.2 we have already shown that

$$
A_{\lambda^{\prime} / 2}(t, q)=b_{\lambda ; 2 m}^{\mathrm{ol}}(q, t)
$$

for $\lambda^{\prime}$ even (or $\lambda^{\prime}$ odd and $\lambda_{1}=2 m$ ). Hence

$$
\left[P_{\lambda}(x ; q, t)\right]\left(x_{1} \cdots x_{n}\right)^{m} P_{m^{n}}^{\left(\mathrm{D}_{n}, \mathrm{D}_{n}\right)}(x ; q, t)=\chi\left(\lambda^{\prime} \text { even }\right) b_{\lambda ; 2 m}^{\mathrm{ol}}(q, t) .
$$

Replacing $m$ by $m / 2$ this proves (4.9a) for even $m$. 
For completeness we remark that the analogue of (4.33) for half-integer $m$ is easily shown to be

$$
\begin{aligned}
{\left[P_{\lambda}(x ; q, t)\right]\left(x_{1} \cdots x_{n}\right)^{m} P_{m^{n}}^{\left(\mathrm{D}_{n}, \mathrm{D}_{n}\right)}(x ; q, t) } \\
=\frac{1}{2}(-1)^{|\lambda|} I_{K}^{(k)}\left(P_{\lambda^{\prime}}\left(x_{1}^{ \pm}, \ldots, x_{k}^{ \pm},-1 ; t, q\right) ; t, q ; 1,-q, \pm q^{1 / 2}\right) \\
\quad+\frac{1}{2}(-1)^{|\lambda|} I_{K}^{(k)}\left(P_{\lambda^{\prime}}\left(x_{1}^{ \pm}, \ldots, x_{k}^{ \pm}, 1 ; t, q\right) ; t, q ;-1, q, \pm q^{1 / 2}\right),
\end{aligned}
$$

where $k=m-1 / 2$. By Theorem 3.5 this again implies that

$$
\left[P_{\lambda}(x ; q, t)\right]\left(x_{1} \cdots x_{n}\right)^{m} P_{m^{n}}^{\left(\mathrm{D}_{n}, \mathrm{D}_{n}\right)}(x ; q, t)=\chi\left(\lambda^{\prime} \text { even }\right) b_{\lambda ; 2 m}^{\mathrm{ol}}(q, t) .
$$

Of course, as noted above, this result follows more simply by a degree argument.

Proof of Theorem 4.7. By (2.54) and (4.17) we must prove that

$$
f_{\lambda}^{(m)}\left(0, t ; 0,0, t_{2}, t_{3}\right)=h_{\lambda}^{(2 m)}\left(t_{2}, t_{3} ; t\right)
$$

for $\lambda \subset(2 m)^{n}$. By (4.18),

$$
f_{\lambda}^{(m)}\left(0, t ; 0,0, t_{2}, t_{3}\right)=(-1)^{|\lambda|} I_{K}^{(m)}\left(P_{\lambda^{\prime}}(t, 0) ; t, 0 ; 0,0, t_{2}, t_{3}\right) .
$$

The integral on the right can be evaluated thanks to Theorem 3.7 with $(n, q, \mu)$ replaced by $\left(m, t, \lambda^{\prime}\right)$. Hence

$$
f_{\lambda}^{(m)}\left(0, t ; 0,0, t_{2}, t_{3}\right)=(-1)^{|\lambda|} h_{\lambda}\left(-t_{2},-t_{3} ; t\right)=h_{\lambda}^{(2 m)}\left(t_{2}, t_{3} ; t\right),
$$

where the second equality follows from definition (2.8) and

$$
(-1)^{|\lambda|}=\prod_{\substack{i \geqslant 1 \\ i \text { odd }}}(-1)^{m_{i}(\lambda)} .
$$

A proof of (4.15) (the large- $m$ limit of Theorem 4.7) using virtual Koornwinder integrals is due to Venkateswaran [92. Her approach, however, is not a limiting version of ours. Crucial difference is that Venkateswaran stays within the $t$-world, whereas we have applied the virtual Koornwinder integral (3.20) over $P_{\lambda}(0, q)$.

Proof of Theorem 4.8. As in earlier proofs we replace $m$ by $2 m$. From (2.61) and (4.20) it follows that we must prove

$$
f_{\lambda}^{(m)}\left(0, t ; t_{2}, 0\right)=h_{\lambda}^{(2 m)}\left(t_{2} ; t\right)
$$

for $\lambda \subset(2 m)^{n}$ and $m$ a nonnegative integer or half-integer. For $m$ an integer, (4.36) is the $t_{3}=-1$ case of (4.34), and in the remainder we assume $m$ is a half-integer.

We will not apply Proposition 4.10 as it is not suitable for taking the $q \rightarrow 0$ limit. Instead we take that limit in (4.28). Then

$$
f_{\lambda}^{(m)}\left(0, t ; t_{2}, t_{3}\right)=(-1)^{|\lambda|} I_{K}^{(k)}\left(P_{\lambda^{\prime}}\left(x_{1}^{ \pm}, \ldots, x_{k}^{ \pm},-1 ; t, 0\right) ; t, 0 ; 0,0, t_{2}, t_{3}\right),
$$


where $k=m-1 / 2$. By the branching rule (2.28) and relation (2.29) this becomes

$$
f_{\lambda}^{(m)}\left(0, t ; t_{2}, t_{3}\right)=\sum_{\mu \subset \lambda}(-1)^{|\mu|} \psi_{\lambda / \mu}^{\prime}(0, t) I_{K}^{(k)}\left(P_{\mu^{\prime}}(t, 0) ; t, 0 ; 0,0, t_{2}, t_{3}\right) .
$$

The virtual Koornwinder integral on the right can be computed by Theorem 3.7 with $(n, q, \mu)$ replaced by $\left(k, t, \mu^{\prime}\right)$. Hence

$$
f_{\lambda}^{(m)}\left(0, t ; t_{2}, t_{3}\right)=\sum_{\substack{\mu \subset \lambda \\ \mu_{1} \leqslant 2 k}}(-1)^{|\mu|} \psi_{\lambda / \mu}^{\prime}(0, t) h_{\mu}^{(2 k)}\left(-t_{2},-t_{3} ; t\right) .
$$

We do not know how to evaluate this in closed form for arbitrary $t_{3}$, but for $t_{3}=0$ it follows from (2.8) that

$$
h_{\mu}^{(2 k)}\left(-t_{2}, 0 ; t\right)=t_{2}^{\operatorname{odd}(\mu)} .
$$

Also using that $(-1)^{|\mu|}=(-1)^{\operatorname{odd}(\mu)}$ we find

$$
f_{\lambda}^{(m)}\left(0, t ; t_{2}, 0\right)=\sum_{\substack{\mu \subset \lambda \\ \mu_{1} \leqslant 2 m-1}}\left(-t_{2}\right)^{\operatorname{odd}(\mu)} \psi_{\lambda / \mu}^{\prime}(0, t) .
$$

Since $\psi_{\lambda / \mu}^{\prime}(0, t)$ is the $e$-Pieri coefficient for ordinary Hall-Littlewood polynomials we have [64, page 215]

$$
\psi_{\lambda / \mu}^{\prime}(0, t)=\prod_{i \geqslant 1}\left[\begin{array}{c}
\lambda_{i}^{\prime}-\lambda_{i+1}^{\prime} \\
\lambda_{i}^{\prime}-\mu_{i}^{\prime}
\end{array}\right]_{t}
$$

Therefore

$$
f_{\lambda}^{(m)}\left(0, t ; t_{2}, 0\right)=\sum_{\substack{\mu \subset \lambda \\
\mu_{1} \leqslant 2 m-1}}\left(-t_{2}\right)^{\operatorname{odd}(\mu)} \prod_{i=1}^{2 m-1}\left[\begin{array}{c}
\lambda_{i}^{\prime}-\lambda_{i+1}^{\prime} \\
\lambda_{i}^{\prime}-\mu_{i}^{\prime}
\end{array}\right]_{t}
$$

Writing $\mu_{i}^{\prime}=\lambda_{i}^{\prime}-k_{i}$ and using (3.25) with $(\mu, \nu, n) \mapsto\left(\mu^{\prime}, \lambda^{\prime}, m\right)$, we finally obtain

$$
\begin{aligned}
f_{\lambda}^{(m)}\left(0, t ; t_{2}, 0\right) & =\left(-t_{2}\right)^{\operatorname{odd}(\lambda)} \prod_{i=1}^{2 m-1} \sum_{k_{i}=0}^{m_{i}(\lambda)}\left(-t_{2}\right)^{(-1)^{i} k_{i}}\left[\begin{array}{c}
m_{i}(\lambda) \\
k_{i}
\end{array}\right]_{t} \\
& =\left(-t_{2}\right)^{\text {odd }(\lambda)} \prod_{\substack{i=1 \\
i \text { odd }}}^{2 m-1} H_{m_{i}(\lambda)}\left(-1 / t_{2} ; t\right) \prod_{\substack{i=1 \\
i \text { even }}}^{2 m-1} H_{m_{i}(\lambda)}\left(-t_{2} ; t\right) \\
& =h_{\lambda}^{(2 m)}\left(t_{2},-1 ; t\right)=h_{\lambda}^{(2 m)}\left(t_{2} ; t\right)
\end{aligned}
$$



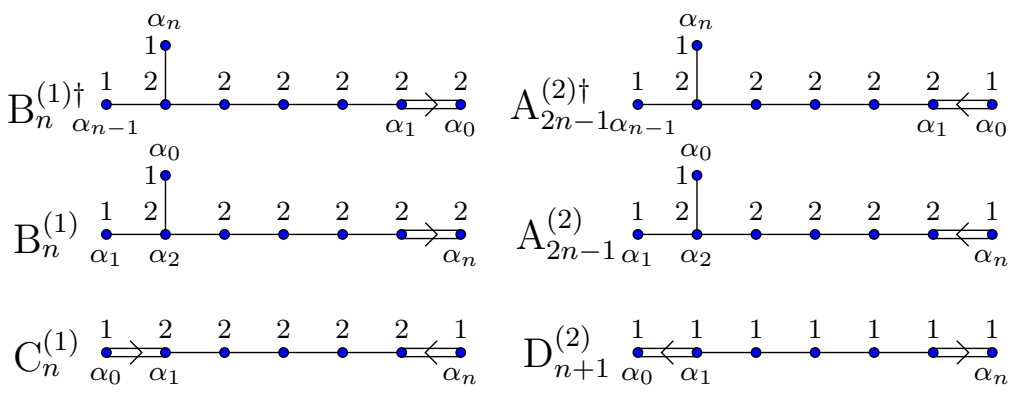
$\mathrm{A}_{2}^{(2) \stackrel{2}{\rightleftarrows} \underset{\alpha_{0}}{\rightleftarrows}}$

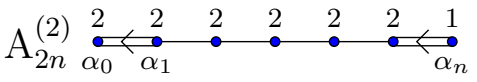

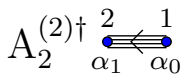

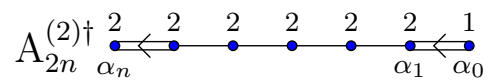

Figure 1. The Dynkin diagrams of the " $\mathrm{BC}_{n}$-type" affine Lie algebras with labelling of vertices by simple roots $\alpha_{0}, \ldots, \alpha_{n}$ and marks $a_{0}, \ldots, a_{n}$.

\section{Applications}

\subsection{Character identities for affine Lie algebras.}

We will only define the bare minimum of notation needed, and for a more comprehensive introduction to the representation theory of affine Lie algebras we refer the reader to [15,61].

We will be concerned with affine Lie algebras $\mathfrak{g}$ of " $\mathrm{BC}_{n}$ type", that is, $\mathrm{B}_{n}^{(1)}, \mathrm{C}_{n}^{(1)}, \mathrm{A}_{2 n-1}^{(2)}, \mathrm{A}_{2 n}^{(2)}$ and $\mathrm{D}_{n+1}^{(2)}$, which are the algebras whose classical part (at least in standard labelling) is one of $\mathrm{B}_{n}$ or $\mathrm{C}_{n}$. The relevant Dynkin diagrams are shown in Figure 5.1, For $\mathrm{B}_{n}^{(1)}, \mathrm{A}_{2 n-1}^{(2)}$ and $\mathrm{A}_{2 n}^{(2)}$ we also use the nonstandard labelling of simple roots (indicated by the customary $\dagger$ ) obtained by mapping $\alpha_{i} \mapsto \alpha_{n-i}$ for $0 \leqslant i \leqslant n$. Apart from the simple roots $\alpha_{0}, \ldots, \alpha_{n}$ and fundamental weights $\Lambda_{0}, \ldots, \Lambda_{n}$ we need the null root $\delta$ given by $\delta=$ $\sum_{i=0}^{n} a_{i} \alpha_{i}$, with the $a_{i}$ the marks of $\mathfrak{g}$. We are interested in representations of $\mathfrak{g}$ known as integrable highest-weight modules. If $P_{+}$is the set of dominant integral weights $P_{+}=\sum_{i=0}^{n} \mathbb{Z}_{\geqslant 0} \Lambda_{i}$ then these modules are indexed by $\Lambda \in P_{+}$, and will be denoted by $V(\Lambda)$ in the following. The character of $V(\Lambda)$ can be computed in closed form by the Weyl-Kac formula:

$$
\operatorname{ch} V(\Lambda)=\frac{\sum_{w \in W} \operatorname{sgn}(w) \mathrm{e}^{w(\Lambda+\rho)-\rho}}{\prod_{\alpha>0}\left(1-\mathrm{e}^{-\alpha}\right)^{\operatorname{mult}(\alpha)}} .
$$


Here $W$ is the Weyl group of $\mathfrak{g}, \operatorname{sgn}(w)$ the signature of $w \in W, \rho=\Lambda_{0}+\cdots+\Lambda_{n}$ the Weyl vector, and mult $(\alpha)$ the multiplicity of $\alpha$. In the denominator, the product runs over the positive roots of $\mathfrak{g}$.

Below we prove combinatorial character formulas for

$$
\chi_{m}(\mathfrak{g}):=\mathrm{e}^{-m \Lambda_{0}} \operatorname{ch} V\left(m \Lambda_{0}\right)
$$

for $\mathfrak{g}$ one of $\mathrm{B}_{n}^{(1)}, \mathrm{B}_{n}^{(1) \dagger}, \mathrm{C}_{n}^{(1)}, \mathrm{A}_{2 n-1}^{(2)}, \mathrm{A}_{2 n-1}^{(2) \dagger}, \mathrm{A}_{2 n}^{(2)}, \mathrm{A}_{2 n}^{(2) \dagger}, \mathrm{D}_{n+1}^{(2)}$. Since the diagrams of $\mathrm{C}_{n}^{(1)}$ and $\mathrm{D}_{n+1}^{(2)}$ are the same when read from left to right as from right to left, these two algebras occur only once in the above list. For $\mathrm{A}_{2 n-1}^{(2) \dagger}, \mathrm{A}_{2 n}^{(2) \dagger}$ and $\mathrm{D}_{n+1}^{(2)}$, however, we obtain two distinct formulas, making a total of eleven character formulas.

Recall that $P_{\lambda}^{(R)}$ denotes a Hall-Littlewood polynomial of type $R$. Also recall the definition of $X$ in (1.5), which may be written plethystically as

$$
X=\left(x_{1}+x_{1}^{-1}+\cdots+x_{n}+x_{n}^{-1}\right) \frac{1-t^{N}}{1-t} .
$$

We complement this with

$$
\begin{aligned}
\bar{X} & :=\left(x_{1}^{ \pm}, t x_{1}^{ \pm}, \ldots, t^{N-1} x_{1}^{ \pm}, \ldots \ldots, x_{n-1}^{ \pm}, t x_{n-1}^{ \pm}, \ldots, t^{N-1} x_{n-1}^{ \pm}, 1, t, \ldots, t^{N-1}\right) \\
& =\left(x_{1}+x_{1}^{-1}+\cdots+x_{n-1}+x_{n-1}^{-1}+1\right) \frac{1-t^{N}}{1-t}
\end{aligned}
$$

Finally, in each of the formulas below $m_{0}(\lambda):=\infty$.

Theorem 5.1. Let

$$
x_{i}:=\mathrm{e}^{-\alpha_{i}-\cdots-\alpha_{n-1}-\alpha_{n} / 2} \quad(1 \leqslant i \leqslant n), \quad t:=\mathrm{e}^{-\delta},
$$

and let $m$ and $n$ be positive integers. Then

$$
\begin{aligned}
\chi_{m}\left(\mathrm{C}_{n}^{(1)}\right) & =\lim _{\substack{N \rightarrow \infty\\
}} t^{m n N^{2}} P_{m^{2 n N}}^{\left(\mathrm{C}_{2 n N}\right)}\left(t^{1 / 2} X ; t, 0\right) \\
& =\sum_{\substack{\lambda \text { even } \\
\lambda_{1} \leqslant 2 m}} t^{|\lambda| / 2} P_{\lambda}^{\prime}\left(x_{1}^{ \pm}, \ldots, x_{n}^{ \pm} ; t\right) \\
\chi_{m}\left(\mathrm{~A}_{2 n-1}^{(2)}\right) & =\left(t ; t^{2}\right)_{\infty} \lim _{N \rightarrow \infty} t^{\frac{1}{2} m n N^{2}} P_{\left(\frac{m}{2}\right)^{2 n N}}^{\left(\mathrm{D}_{2 n N}\right)}\left(t^{1 / 2} X ; t\right) \\
& =\sum_{\substack{\lambda^{\prime} \text { even } \\
\lambda_{1} \leqslant m}} t^{|\lambda| / 2} P_{\lambda}^{\prime}\left(x_{1}^{ \pm}, \ldots, x_{n}^{ \pm} ; t\right) \prod_{i=0}^{m-1}\left(t ; t^{2}\right)_{m_{i}(\lambda) / 2}
\end{aligned}
$$


and

$$
\begin{aligned}
& \chi_{m}\left(\mathrm{~A}_{2 n}^{(2)}\right)=\lim _{N \rightarrow \infty} t^{\frac{1}{2} m n N^{2}} P_{\left(\frac{m}{2}\right)^{2 n N}}^{\left(\mathrm{B}_{2 n N}\right)}\left(t^{1 / 2} X ; t, 0\right) \\
& =\sum_{\substack{\lambda \\
\lambda_{1} \leqslant m}} t^{|\lambda| / 2} P_{\lambda}^{\prime}\left(x_{1}^{ \pm}, \ldots, x_{n}^{ \pm} ; t\right) .
\end{aligned}
$$

Theorem 5.2. Let

$$
x_{i}:=\mathrm{e}^{-\alpha_{i}-\cdots-\alpha_{n-1}+\left(\alpha_{n-1}-\alpha_{n}\right) / 2} \quad(1 \leqslant i \leqslant n), \quad t:=\mathrm{e}^{-\delta},
$$

and let $m$ and $n$ be positive integers. Then

$$
\begin{aligned}
\chi_{m}\left(\mathrm{~A}_{2 n-1}^{(2) \dagger}\right) & =\left(t ; t^{2}\right)_{\infty} \lim _{N \rightarrow \infty} t^{m n N^{2}} P_{m^{2 n N}}^{\left(\mathrm{C}_{2 n N}\right)}\left(t^{1 / 2} X ; t, t\right) \\
= & \sum_{\substack{\lambda \\
\lambda_{1} \leqslant 2 m}}^{\prime} t^{(|\lambda|+\operatorname{odd}(\lambda)) / 2} P_{\lambda}^{\prime}\left(x_{1}^{ \pm}, \ldots, x_{n}^{ \pm} ; t\right) \prod_{i=0}^{2 m-1}\left(t ; t^{2}\right)_{\left\lceil m_{i}(\lambda) / 2\right\rceil},
\end{aligned}
$$

where the prime in the sum on the right denotes the restriction " $m_{i}(\lambda)$ is even for $i=1,3, \ldots, 2 m-1$ ".

Theorem 5.3. Let

$$
x_{i}:=\mathrm{e}^{-\alpha_{i}-\cdots-\alpha_{n}} \quad(1 \leqslant i \leqslant n),
$$

and let $m$ and $n$ be positive integers. Then

$$
\begin{aligned}
& \chi_{m}\left(\mathrm{~A}_{2 n}^{(2) \dagger}\right)=\lim _{N \rightarrow \infty} t^{m n N^{2}} P_{m^{2 n N}}^{\left(\mathrm{BC}_{2 n N}\right)}\left(t^{1 / 2} X ; t, 0,-t^{1 / 2}\right) \\
& =\sum_{\substack{\lambda \\
\lambda_{1} \leqslant 2 m}} t^{(|\lambda|+\operatorname{odd}(\lambda)) / 2} P_{\lambda}^{\prime}\left(x_{1}^{ \pm}, \ldots, x_{n}^{ \pm} ; t\right),
\end{aligned}
$$

where $t:=\mathrm{e}^{-\delta}$, and

$$
\begin{aligned}
\chi_{m}\left(\mathrm{D}_{n+1}^{(2)}\right) & =\left(-t^{1 / 2} ; t^{1 / 2}\right)_{\infty} \lim _{N \rightarrow \infty} t^{\frac{1}{2} m n N^{2}} P_{\left(\frac{m}{2}\right)^{2 n N}}^{\left(\mathrm{B}_{2 n N}\right)}\left(t^{1 / 2} X ; t,-t^{1 / 2}\right) \\
& =\sum_{\substack{\lambda \\
\lambda_{1} \leqslant m}} t^{|\lambda| / 2} P_{\lambda}^{\prime}\left(x_{1}^{ \pm}, \ldots, x_{n}^{ \pm} ; t\right) \prod_{i=0}^{m-1}\left(-t^{1 / 2} ; t^{1 / 2}\right)_{m_{i}(\lambda)},
\end{aligned}
$$

where $t^{1 / 2}:=\mathrm{e}^{-\delta}$.

The identity (5.5) without the limiting expression in the middle is [5. Theorem 1.1; (1.4a)], (5.7), which is (1.4) from the introduction, extends [5, Theorem 1.1; (1.4b)] from integer to half-integer values of $m$ and (5.11) in the same way extends [5, Theorem 5.4]. The identity [5.10, again without expression on the right, is [5, Theorem 5.3].

In each of the remaining formulas $\mathrm{e}^{-\alpha_{n}}$ is specialised. 
Theorem 5.4. Let

$$
x_{i}:=-\mathrm{e}^{-\alpha_{i}-\cdots-\alpha_{n}} \quad(1 \leqslant i \leqslant n-1), \quad t:=\mathrm{e}^{-\delta},
$$

and specialise $\mathrm{e}^{-\alpha_{n}} \mapsto-1$. Then, for $m$ and $n$ positive integers,

$$
\begin{aligned}
& \chi_{m}\left(\mathrm{~A}_{2 n}^{(2) \dagger}\right)=\lim _{N \rightarrow \infty} t^{\frac{1}{2} m(2 n-1) N^{2}} P_{m^{(2 n-1) N}}^{\left(\mathrm{C}_{(2 n-1) N}\right)}\left(t^{1 / 2} \bar{X} ; t, 0\right) \\
& =\sum_{\substack{\lambda \text { even } \\
\lambda_{1} \leqslant 2 m}} t^{|\lambda| / 2} P_{\lambda}^{\prime}\left(x_{1}^{ \pm}, \ldots, x_{n-1}^{ \pm}, 1 ; t\right)
\end{aligned}
$$

and

$$
\begin{aligned}
\chi_{m}\left(\mathrm{~B}_{n}^{(1)}\right) & =\left(t ; t^{2}\right)_{\infty} \lim _{N \rightarrow \infty} t^{\frac{1}{4} m(2 n-1) N^{2}} P_{\left(\frac{m}{2}\right)(2 n-1) N}^{\left(\mathrm{D}_{(2 n-1) N}\right)}\left(t^{1 / 2} \bar{X} ; t\right) \\
& =\sum_{\substack{\lambda^{\prime} \text { even } \\
\lambda_{1} \leqslant m}} t^{|\lambda| / 2} P_{\lambda}^{\prime}\left(x_{1}^{ \pm}, \ldots, x_{n-1}^{ \pm}, 1 ; t\right) \prod_{i=0}^{m-1}\left(t ; t^{2}\right)_{m_{i}(\lambda) / 2}
\end{aligned}
$$

Theorem 5.5. Let

$$
x_{i}:=-\mathrm{e}^{-\alpha_{i}-\cdots-\alpha_{n}} \quad(1 \leqslant i \leqslant n-1), \quad t^{1 / 2}:=-\mathrm{e}^{-\delta},
$$

and specialise $\mathrm{e}^{-\alpha_{n}} \mapsto-1$. Then, for $m$ and $n$ positive integers,

$$
\begin{aligned}
& \chi_{m}\left(\mathrm{D}_{n+1}^{(2)}\right)=\lim _{N \rightarrow \infty} t^{\frac{1}{4} m(2 n-1) N^{2}} P_{\left(\frac{m}{2}\right)^{(2 n-1) N}}^{\left(\mathrm{B}_{(2 n-1) N}\right)}\left(t^{1 / 2} \bar{X} ; t, 0\right) \\
& =\sum_{\substack{\lambda \\
\lambda_{1} \leqslant m}} t^{|\lambda| / 2} P_{\lambda}^{\prime}\left(x_{1}^{ \pm}, \ldots, x_{n-1}^{ \pm}, 1 ; t\right),
\end{aligned}
$$

Theorem 5.6. Let

$$
x_{i}:=\mathrm{e}^{-\alpha_{i}-\cdots-\alpha_{n-1}+\left(\alpha_{n-1}-\alpha_{n}\right) / 2} \quad(1 \leqslant i \leqslant n-1), \quad t:=\mathrm{e}^{-\delta},
$$

and specialise $\mathrm{e}^{-\alpha_{n}} \mapsto \mathrm{e}^{-\alpha_{n-1}}$. Then, for $m$ and $n$ positive integers,

$$
\begin{aligned}
\chi_{m}\left(\mathrm{~B}_{n}^{(1) \dagger}\right) & =\left(-t^{1 / 2} ; t^{1 / 2}\right)_{\infty} \lim _{N \rightarrow \infty} t^{\frac{1}{4} m(2 n-1) N^{2}} P_{\left(\frac{m}{2}\right)^{(2 n-1) N}}^{\left(\mathrm{B}_{(2 n-1) N}\right)}\left(t^{1 / 2} \bar{X} ; t,-t^{1 / 2}\right) \\
& =\sum_{\substack{\lambda \\
\lambda_{1} \leqslant m}} t^{|\lambda| / 2} P_{\lambda}^{\prime}\left(x_{1}^{ \pm}, \ldots, x_{n-1}^{ \pm}, 1 ; t\right) \prod_{i=0}^{m-1}\left(-t^{1 / 2} ; t^{1 / 2}\right)_{m_{i}(\lambda)}
\end{aligned}
$$

and

$$
\begin{aligned}
\chi_{m}\left(\mathrm{~A}_{2 n-1}^{(2) \dagger}\right) & =\lim _{\substack{N \rightarrow \infty\\
}} t^{m(n-1 / 2) N^{2}} P_{m^{(2 n-1) N}}^{\left(\mathrm{BC}_{(2 n-1) N}\right)}\left(t^{1 / 2} \bar{X} ; t, 0,-t^{1 / 2}\right) \\
& =\sum_{\substack{\lambda \\
\lambda_{1} \leqslant 2 m}} t^{(|\lambda|+\operatorname{odd}(\lambda)) / 2} P_{\lambda}^{\prime}\left(x_{1}^{ \pm}, \ldots, x_{n-1}^{ \pm}, 1 ; t\right) .
\end{aligned}
$$


The rest of this section is devoted to a proof of these eleven formulas.

Recall the Vandermonde determinants of type $\mathrm{B}_{n}, \mathrm{C}_{n}$ and $\mathrm{D}_{n}$ given in (2.56) (2.63) and (2.65).

Proposition 5.7. For $x=\left(x_{1}, \ldots, x_{n}\right), m$ a positive integer and $N$ a nonnegative integer,

$$
\begin{aligned}
& \sum_{\substack{\lambda \\
\lambda_{1} \leqslant 2 m}} t^{|\lambda| / 2} h_{\lambda}^{(2 m)}\left(t_{2}, t_{3} ; t\right) P_{\lambda}\left(\left[x \frac{1-t^{N}}{1-t}\right] ; t\right) \\
& =\left(x_{1} \cdots x_{n}\right)^{m N} t^{m n N^{2} / 2} P_{m^{n N}}^{\left(\mathrm{BC}_{n N}\right)}\left(\left[t^{1 / 2} x \frac{1-t^{N}}{1-t}\right] ; t, t_{2}, t_{3}\right) \\
& =\frac{\prod_{i=1}^{n}\left(t^{1 / 2} t_{2} x_{i}, t^{1 / 2} t_{3} x_{i} ; t\right)_{N}}{\prod_{i, j=1}^{n}\left(t x_{i} x_{j} ; t\right)_{N}} \prod_{1 \leqslant i<j \leqslant n}\left(t x_{i} x_{j} ; t\right)_{2 N} \\
& \quad \sum_{r_{1}, \ldots, r_{n} \geqslant 0} \frac{\Delta_{\mathrm{C}}\left(x t^{r}\right)}{\Delta_{\mathrm{C}}(x)} \prod_{i=1}^{n} \frac{\left(t^{1 / 2} t_{2}^{-1} x_{i}, t^{1 / 2} t_{3}^{-1} x_{i} ; t\right)_{r_{i}}}{\left(t^{1 / 2} t_{2} x_{i}, t^{1 / 2} t_{3} x_{i} ; t\right)_{r_{i}}}\left(t_{2} t_{3}\right)^{r_{i}}\left(x_{i}^{2} t^{r_{i}}\right)^{m r_{i}} \\
& \times \prod_{i, j=1}^{n} \frac{\left(t^{-N} x_{i} / x_{j}, x_{i} x_{j} ; t\right)_{r_{i}}}{\left(t x_{i} / x_{j}, t^{N+1} x_{i} x_{j} ; t\right)_{r_{i}}}
\end{aligned}
$$

Remark 5.8. A more general hypergeometric identity than (5.20) holds, obtained by replacing

$$
x \mapsto t^{1 / 2}\left(x_{1} \frac{1-t^{N_{1}}}{1-t}+x_{2} \frac{1-t^{N_{2}}}{1-t}+\cdots+x_{n} \frac{1-t^{N_{n}}}{1-t}\right)
$$

in (4.14). From a hypergeometric point of view this more general identity, which on the right features the $\mathrm{C}_{n}$ hypergeometric series

$$
\begin{aligned}
\sum_{r_{1}, \ldots, r_{n} \geqslant 0} \frac{\Delta_{\mathrm{C}}\left(x t^{r}\right)}{\Delta_{\mathrm{C}}(x)} \prod_{i=1}^{n} \frac{\left(t^{1 / 2} t_{2}^{-1} x_{i}, t^{1 / 2} t_{3}^{-1} x_{i} ; t\right)_{r_{i}}}{\left(t^{1 / 2} t_{2} x_{i}, t^{1 / 2} t_{3} x_{i} ; t\right)_{r_{i}}}\left(t_{3}\right)^{r_{i}}\left(x_{i}^{2} t^{r_{i}}\right)^{m r_{i}} \\
\times \prod_{i, j=1}^{n} \frac{\left(t^{-N_{j}} x_{i} / x_{j}, x_{i} x_{j} ; t\right)_{r_{i}}}{\left(t x_{i} / x_{j}, t^{N_{j}+1} x_{i} x_{j} ; t\right)_{r_{i}}} t^{N_{j} r_{i}}
\end{aligned}
$$

is more natural. For our purposes, however, we do not require this greater degree of generality.

Proof. Identity (5.20) follows from (4.14) by the substitution

$$
x \mapsto t^{1 / 2}\left(x_{1}, x_{1} t, \ldots, x_{1} t^{N-1}, \ldots \ldots, x_{n}, x_{n} t, \ldots, x_{n} t^{N-1}\right)=t^{1 / 2} x \frac{1-t^{N}}{1-t}
$$


(so that, implicitly, $n \mapsto n N$ ). The two left-most expressions immediately follow from (5.20), but to show equality with the hypergeometric sum on the right some work is required.

First we use Lemma 2.4 to trade the right-hand side of (4.14) for

$$
\sum_{\varepsilon \in\{ \pm 1\}^{n}} \Phi\left(x^{\varepsilon} ; t_{2}, t_{3} ; t\right) \prod_{i=1}^{n} x_{i}^{\left(1-\varepsilon_{i}\right) m} .
$$

Next we observe that $\Phi\left(x^{\varepsilon} ; t_{2}, t_{3} ; t\right)$ contains the factor

$$
\prod_{1 \leqslant i<j \leqslant n}\left(1-t x_{i}^{\varepsilon_{i}} x_{j}^{\varepsilon_{j}}\right)
$$

which vanishes if there exists an $i(1 \leqslant i \leqslant n-1)$ such that

$$
t x_{i}^{\varepsilon_{i}} x_{i+1}^{\varepsilon_{i+1}}=1 \text {. }
$$

Therefore, by the substitution (5.21) the summand vanishes if for some $i$, $\left(\varepsilon_{i}, \varepsilon_{i+1}\right)=(1,-1)$ while $\left(x_{i}, x_{i+1}\right)$ is mapped to $\left(x_{u} t^{p}, x_{u} t^{p+1}\right)$ for some $u$ and $p$. In other words, the only sequences $\varepsilon$ that yield a non-vanishing summand are of the form

$$
\varepsilon=(\underbrace{-1, \ldots,-1}_{r_{1} \text { times }}, \underbrace{1, \ldots, 1}_{N-r_{1} \text { times }}, \underbrace{-1, \ldots,-1}_{r_{2} \text { times }}, \underbrace{1, \ldots, 1}_{N-r_{2} \text { times }}, \ldots, \underbrace{-1, \ldots,-1}_{r_{n} \text { times }}, \underbrace{1, \ldots, 1}_{N-r_{n} \text { times }}) .
$$

The $r_{i}$ are exactly the summation indices of (5.20). The rest of the proof is tedious but elementary and left to the reader.

Replacing

$$
x \mapsto\left(x_{1}, y_{1}, \ldots, x_{n}, y_{n}\right)
$$

in (5.20) (so that $n \mapsto 2 n$ ), and then using [5, Proposition 5.1] to take the limit $y_{i} \mapsto x_{i}^{-1}$ for all $1 \leqslant i \leqslant n$, we obtain the following corollary of Proposition 5.7 .

Corollary 5.9. Let $m$ a positive integer, $N$ a nonnegative integer and $X$ the alphabet (1.5). Then

$$
\begin{aligned}
\sum_{\substack{\lambda \\
\lambda_{1} \leqslant 2 m}} t^{|\lambda| / 2} h_{\lambda}^{(2 m)}\left(t_{2}, t_{3} ; t\right) P_{\lambda}(X ; t) & t^{m n N^{2}} P_{m^{2 n N}}^{\left(\mathrm{BC}_{2 n N}\right)}\left(t^{1 / 2} X ; t, t_{2}, t_{3}\right) \\
= & \prod_{i=1}^{n} \frac{\left(t^{1 / 2} t_{2} x_{i}^{ \pm}, t^{1 / 2} t_{3} x_{i}^{ \pm} ; t\right)_{N}}{\left(t x_{i}^{ \pm 2} ; t\right)_{N}}\left[\begin{array}{c}
2 N \\
N
\end{array}\right]_{t} \prod_{1 \leqslant i<j \leqslant n} \frac{\left(t x_{i}^{ \pm} x_{j}^{ \pm} ; t\right)_{2 N}}{\left(t x_{i}^{ \pm} x_{j}^{ \pm} ; t\right)_{N}^{2}} \\
& \times \sum_{r \in \mathbb{Z}^{n}} \frac{\Delta_{\mathrm{C}}\left(x t^{r}\right)}{\Delta_{\mathrm{C}}(x)} \prod_{i=1}^{n} \frac{\left(t^{1 / 2} t_{2}^{-1} x_{i}, t^{1 / 2} t_{3}^{-1} x_{i} ; t\right)_{r_{i}}\left(t_{2} t_{3}\right)^{r_{i}}\left(x_{i}^{2} t^{r_{i}}\right)^{m r_{i}}}{\left(t^{1 / 2} t_{2} x_{i}, t^{1 / 2} t_{3} x_{i} ; t\right)_{r_{i}}}
\end{aligned}
$$




$$
\times \prod_{i, j=1}^{n} \frac{\left(t^{-N} x_{i} x_{j}^{ \pm} ; t\right)_{r_{i}}}{\left(t^{N+1} x_{i} x_{j}^{ \pm} ; t\right)_{r_{i}}} t^{2 N r_{i}}
$$

Since

$$
\lim _{N \rightarrow \infty} P_{\lambda}(X ; t)=P_{\lambda}\left(\left[\frac{x_{1}^{ \pm}+\cdots+x_{n}^{ \pm}}{1-t}\right] ; t\right)=P_{\lambda}^{\prime}\left(x_{1}^{ \pm}, \cdots x_{n}^{ \pm} ; t\right),
$$

the above corollary is a bounded analogue of [5, Theorem 5.2; (5.6a)], which states (without the second line) that

$$
\begin{aligned}
& \sum_{\substack{\lambda \\
\lambda_{1} \leqslant 2 m}} t^{|\lambda| / 2} h_{\lambda}^{(2 m)}\left(t_{2}, t_{3} ; t\right) P_{\lambda}^{\prime}\left(x_{1}^{ \pm}, \ldots, x_{n}^{ \pm} ; t\right) \\
& =\lim _{N \rightarrow \infty} t^{m n N^{2}} P_{m^{2 n N}}^{\left(\mathrm{BC}_{2 n N}\right)}\left(t^{1 / 2} X ; t, t_{2}, t_{3}\right) \\
& =\frac{1}{(t ; t)_{\infty}^{n}} \prod_{i=1}^{n} \frac{\left(t^{1 / 2} t_{2} x_{i}^{ \pm}, t^{1 / 2} t_{3} x_{i}^{ \pm} ; t\right)_{\infty}}{\left(t x_{i}^{ \pm 2} ; t\right)_{\infty}} \prod_{1 \leqslant i<j \leqslant n} \frac{1}{\left(t x_{i}^{ \pm} x_{j}^{ \pm} ; t\right)_{\infty}} \\
& \quad \times \sum_{r \in \mathbb{Z}^{n}} \frac{\Delta_{\mathrm{C}}\left(x t^{r}\right)}{\Delta_{\mathrm{C}}(x)} \prod_{i=1}^{n} \frac{\left(t^{1 / 2} t_{2}^{-1} x_{i}, t^{1 / 2} t_{3}^{-1} x_{i} ; t\right)_{r_{i}}}{\left(t^{1 / 2} t_{2} x_{i}, t^{1 / 2} t_{3} x_{i} ; t\right)_{r_{i}}}\left(t^{-n}\right)^{r_{i}}\left(x_{i}^{2} t^{r_{i}}\right)^{(m+n) r_{i}}
\end{aligned}
$$

for $m$ a positive integer.

If instead of (5.22) we make the substitution

$$
x \mapsto\left(x_{1}, y_{1}, \ldots, x_{n-1}, y_{n-1}, x_{n}\right)
$$

in (5.20) (so that $n \mapsto 2 n-1$ ) and then take the limit $y_{i} \mapsto x_{i}^{-1}$ for all $1 \leqslant i \leqslant n-1$ and $x_{n} \rightarrow 1$ using [5, Proposition 5.1], we obtain a bounded version of $[5 \text {, Theorem } 5.2 ;(5.6 \mathrm{~b})]^{8}$

$$
\begin{aligned}
& \sum_{\substack{\lambda \\
\lambda_{1} \leqslant 2 m}} t^{|\lambda| / 2} h_{\lambda}^{(2 m)}\left(t_{2}, t_{3} ; t\right) P_{\lambda}^{\prime}\left(x_{1}^{ \pm}, \ldots, x_{n-1}^{ \pm}, 1 ; t\right) \\
& =\lim _{N \rightarrow \infty} t^{m(n-1 / 2) N^{2}} P_{m^{(2 n-1) N}}^{\left(\mathrm{BC}_{(2 n-1) N}\right)}\left(t^{1 / 2} \bar{X} ; t, t_{2}, t_{3}\right) \\
& =\frac{1}{(t ; t)_{\infty}^{n}\left(t^{1 / 2} t_{2}, t^{1 / 2} t_{3} ; t\right)_{\infty}} \prod_{i=1}^{n} \frac{\left(t^{1 / 2} t_{2} x_{i}^{ \pm}, t^{1 / 2} t_{3} x_{i}^{ \pm} ; t\right)_{\infty}}{\left(-t x_{i}^{ \pm} ; t\right)_{\infty}\left(t x_{i}^{ \pm 2} ; t^{2}\right)_{\infty}} \\
& \quad \times \prod_{1 \leqslant i<j \leqslant n} \frac{1}{\left(t x_{i}^{ \pm} x_{j}^{ \pm} ; t\right)_{\infty}} \sum_{r \in \mathbb{Z}^{n}}\left(\frac{\Delta_{\mathrm{B}}\left(-x t^{r}\right)}{\Delta_{\mathrm{B}}(-x)}\right.
\end{aligned}
$$

\footnotetext{
${ }^{8}$ Taking $x_{n} \rightarrow t^{1 / 2}$ instead of $x_{n} \rightarrow 1$ yields additional character identities to those of Theorems 5.1 and 5.6 .
} 


$$
\left.\times \prod_{i=1}^{n} \frac{\left(t^{1 / 2} t_{2}^{-1} x_{i}, t^{1 / 2} t_{3}^{-1} x_{i} ; t\right)_{r_{i}}}{\left(t^{1 / 2} t_{2} x_{i}, t^{1 / 2} t_{3} x_{i} ; t\right)_{r_{i}}}\left(-t_{2} t_{3} t^{1 / 2-n}\right)^{r_{i}}\left(x_{i}^{2} t^{r_{i}}\right)^{(m+n-1 / 2) r_{i}}\right),
$$

where $m$ is a positive integer and $x_{n}:=1$.

We are now ready to prove Theorems 5.15.6. Our first two character formulas follow from (5.23) and (5.25) by letting $t_{2}$ and $t_{3}$ tend to zero. The hypergeometric sums on the right can then be identified with $\chi_{m}\left(\mathrm{C}_{n}^{(1)}\right)$ and $\chi_{m}\left(\mathrm{~A}_{2 n}^{(2) \dagger}\right)$ respectively, by [5, Lemmas $2.1 \& 2.3$ ] and [5, Lemma 2.3] (which are simple rewritings of the Weyl-Kac formula for these two affine Lie algebras). In the latter case this identification requires the specialisation $\mathrm{e}^{-\alpha_{n}} \mapsto-1$, corresponding to the condition $x_{n}:=1$ in (5.25). In the $t_{2}, t_{3} \rightarrow 0$ limit the left-hand sides simplifies since $h_{\lambda}^{(2 m)}(0,0 ; t)=\chi(\lambda$ even). We thus obtain (5.5) and (5.13).

Next we specialise $t_{3}=-t^{1 / 2}$ in (5.23) and (5.25). Using

$$
\begin{gathered}
\frac{\Delta_{\mathrm{C}}\left(x t^{r}\right)}{\Delta_{\mathrm{C}}(x)} \prod_{i=1}^{n} \frac{\left(-x_{i} ; t\right)_{r_{i}}}{\left(-t x_{i} ; t\right)_{r_{i}}}=\frac{\Delta_{\mathrm{B}}\left(x t^{r}\right)}{\Delta_{\mathrm{B}}(x)} \\
\frac{\Delta_{\mathrm{B}}\left(-x t^{r}\right)}{\Delta_{\mathrm{B}}(-x)} \prod_{i=1}^{n} \frac{\left(-x_{i} ; t\right)_{r_{i}}}{\left(-t x_{i} ; t\right)_{r_{i}}}=\frac{\Delta_{\mathrm{D}}\left(x t^{r}\right)}{\Delta_{\mathrm{D}}(x)}
\end{gathered}
$$

respectively, this yields

$$
\begin{aligned}
& \sum_{\substack{\lambda \\
\lambda_{1} \leqslant 2 m}} t^{|\lambda| / 2} h_{\lambda}^{(2 m)}\left(t_{2},-t^{1 / 2} ; t\right) P_{\lambda}^{\prime}\left(x_{1}^{ \pm}, \ldots, x_{n}^{ \pm} ; t\right) \\
& =\lim _{N \rightarrow \infty} t^{m n N^{2}} P_{m^{2 n N}}^{\left(\mathrm{BC}_{2 n N}\right)}\left(t^{1 / 2} X ; t, t_{2},-t^{1 / 2}\right) \\
& =\frac{1}{(t ; t)_{\infty}^{n}} \prod_{i=1}^{n} \frac{\left(t^{1 / 2} t_{2} x_{i}^{ \pm} ; t\right)_{\infty}}{\left(t x_{i}^{ \pm} ; t\right)_{\infty}\left(t x_{i}^{ \pm 2} ; t^{2}\right)_{\infty}} \prod_{1 \leqslant i<j \leqslant n} \frac{1}{\left(t x_{i}^{ \pm} x_{j}^{ \pm} ; t\right)_{\infty}} \\
& \quad \times \sum_{r \in \mathbb{Z}^{n}} \frac{\Delta_{\mathrm{B}}\left(x t^{r}\right)}{\Delta_{\mathrm{B}}(x)} \prod_{i=1}^{n} \frac{\left(t^{1 / 2} t_{2}^{-1} x_{i} ; t\right)_{r_{i}}}{\left(t^{1 / 2} t_{2} x_{i} ; t\right)_{r_{i}}}\left(-t_{2} t^{1 / 2-n}\right)^{r_{i}}\left(x_{i}^{2} t^{r_{i}}\right)^{(m+n) r_{i}}
\end{aligned}
$$

and

$$
\begin{aligned}
& \sum_{\substack{\lambda \\
\lambda_{1} \leqslant 2 m}} t^{|\lambda| / 2} h_{\lambda}^{(2 m)}\left(t_{2},-t^{1 / 2} ; t\right) P_{\lambda}^{\prime}\left(x_{1}^{ \pm}, \ldots, x_{n-1}^{ \pm}, 1 ; t\right) \\
& \quad=\lim _{N \rightarrow \infty} t^{m(n-1 / 2) N^{2}} P_{m^{(2 n-1) N}}^{\left(\mathrm{BC}_{(2 n-1) N}\right)}\left(t^{1 / 2} \bar{X} ; t, t_{2},-t^{1 / 2}\right) \\
& =\frac{1}{(t ; t)_{\infty}^{n-1}\left(t^{2} ; t^{2}\right)_{\infty}\left(t^{1 / 2} t_{2} ; t\right)_{\infty}} \prod_{i=1}^{n} \frac{\left(t^{1 / 2} t_{2} x_{i}^{ \pm} ; t\right)_{\infty}}{\left(t x_{i}^{ \pm 2} ; t^{2}\right)_{\infty}} \prod_{1 \leqslant i<j \leqslant n} \frac{1}{\left(t x_{i}^{ \pm} x_{j}^{ \pm} ; t\right)_{\infty}}
\end{aligned}
$$




$$
\times \sum_{r \in \mathbb{Z}^{n}}\left(\frac{\Delta_{\mathrm{D}}\left(x t^{r}\right)}{\Delta_{\mathrm{D}}(x)} \prod_{i=1}^{n} \frac{\left(t^{1 / 2} t_{2}^{-1} x_{i} ; t\right)_{r_{i}}}{\left(t^{1 / 2} t_{2} x_{i} ; t\right)_{r_{i}}}\left(t_{2} t^{1-n}\right)^{r_{i}}\left(x_{i}^{2} t^{r_{i}}\right)^{(m+n-1 / 2) r_{i}}\right),
$$

where $x_{n}:=1$ in the second identity. Taking the $t_{2} \rightarrow 0$ limit using

$$
h_{\lambda}^{(m)}(0, b ; t)=(-b)^{\operatorname{odd}(\lambda)},
$$

and identifying the respective right-hand sides as $\chi_{m}\left(\mathrm{~A}_{2 n}^{(2) \dagger}\right)$ and $\chi_{m}\left(\mathrm{~A}_{2 n-1}^{(2) \dagger}\right)$ by [5, Lemma 2.3] and Lemma A.3, results in (5.10) and (5.19). In particular we note that $x_{n}$ being 1 in (5.28) implies that we must specialise $\mathrm{e}^{-\alpha_{n}} \mapsto \mathrm{e}^{-\alpha_{n-1}}$, see (A.6). In similar manner we specialise $t_{2}=t^{1 / 2}$ in (5.27) (considering (5.28) does not lead to a character identity). Using (5.26b) with $x \mapsto-x$ it follows from Lemma A.3 that the right-hand side simplifies to

$$
(-t ; t)_{\infty} \chi_{m}\left(\mathrm{~A}_{2 n-1}^{(2) \dagger}\right) \text {. }
$$

By (2.8) the Rogers-Szegö polynomial in the summand on the left becomes

$$
h_{\lambda}^{(2 m)}\left(-t^{1 / 2}, t^{1 / 2} ; t\right)=t^{\text {odd }(\lambda) / 2} \prod_{\substack{i=1 \\ i \text { odd }}}^{m-1} H_{m_{i}(\lambda)}(-1 ; t) \prod_{\substack{i=1 \\ i \text { even }}}^{m-1} H_{m_{i}(\lambda)}(-t ; t) .
$$

Using (2.11b) and (2.11c) this yields

$$
h_{\lambda}^{(2 m)}\left(-t^{1 / 2}, t^{1 / 2} ; t\right)=\prod_{i=1}^{2 m-1}\left(t ; t^{2}\right)_{\lceil m / 2\rceil}
$$

when $m_{i}(\lambda)$ even for all $i=1,3, \ldots, 2 m-1$ and zero otherwise. Finally noting that $(-t ; t)_{\infty}\left(t ; t^{2}\right)_{\infty}=1$, Theorem 5.2 follows. There is one further specialisation of (5.27) and (5.28) that leads to character identities, namely $t_{2}=-1$. We will consider this case as part of a more general treatment of (5.23) and (5.25) for $t_{3}=-1$.

Recalling that Theorem 4.8 extends the $t_{3}=-1$ case of Theorem 4.7 to half-integer values of $m$, the $t_{3}=-1$ specialisations of (5.23) and (5.25) lead to

$$
\begin{aligned}
& \sum_{\substack{\lambda \\
\lambda_{1} \leqslant m}} t^{|\lambda| / 2} h_{\lambda}^{(m)}\left(t_{2} ; t\right) P_{\lambda}^{\prime}\left(x_{1}^{ \pm}, \ldots, x_{n}^{ \pm} ; t\right) \\
& =\lim _{N \rightarrow \infty} t^{m n N^{2} / 2} P_{(m / 2)^{2 n N}}^{\left(\mathrm{B}_{2 n N}\right)}\left(t^{1 / 2} X ; t, t_{2}\right) \\
& =\frac{1}{(t ; t)_{\infty}^{n}} \prod_{i=1}^{n} \frac{\left(t^{1 / 2} t_{2} x_{i}^{ \pm} ; t\right)_{\infty}}{\left(t^{1 / 2} x_{i}^{ \pm} ; t\right)_{\infty}\left(t^{2} x_{i}^{ \pm 2} ; t^{2}\right)_{\infty}} \prod_{1 \leqslant i<j \leqslant n} \frac{1}{\left(t x_{i}^{ \pm} x_{j}^{ \pm} ; t\right)_{\infty}} \\
& \quad \times \sum_{r \in \mathbb{Z}^{n}} \frac{\Delta_{\mathrm{C}}\left(x t^{r}\right)}{\Delta_{\mathrm{C}}(x)} \prod_{i=1}^{n} \frac{\left(t^{1 / 2} t_{2}^{-1} x_{i} ; t\right)_{r_{i}}}{\left(t^{1 / 2} t_{2} x_{i} ; t\right)_{r_{i}}}\left(-t_{2} t^{-n}\right)^{r_{i}}\left(x_{i}^{2} t^{r_{i}}\right)^{(m+2 n) r_{i} / 2}
\end{aligned}
$$


and

$$
\begin{aligned}
& \sum_{\substack{\lambda \\
\lambda_{1} \leqslant m}} t^{|\lambda| / 2} h_{\lambda}^{(m)}\left(t_{2} ; t\right) P_{\lambda}^{\prime}\left(x_{1}^{ \pm}, \ldots, x_{n-1}^{ \pm}, 1 ; t\right) \\
& =\lim _{N \rightarrow \infty} t^{m(2 n-1) N^{2} / 4} P_{(m / 2)(2 n-1) N}^{\left(\mathrm{B}_{(2 n-1) N}\right)}\left(t^{1 / 2} \bar{X} ; t, t_{2}\right) \\
& =\frac{1}{(t ; t)_{\infty}^{n}\left(t^{1 / 2} t_{2},-t^{1 / 2} ; t\right)_{\infty}} \prod_{i=1}^{n} \frac{\left(t^{1 / 2} t_{2} x_{i}^{ \pm} ; t\right)_{\infty}}{\left(t^{1 / 2} x_{i}^{ \pm},-t x_{i}^{ \pm} ; t\right)_{\infty}} \prod_{1 \leqslant i<j \leqslant n} \frac{1}{\left(t x_{i}^{ \pm} x_{j}^{ \pm} ; t\right)_{\infty}} \\
& \quad \times \sum_{r \in \mathbb{Z}^{n}} \frac{\Delta_{\mathrm{B}}\left(-x t^{r}\right)}{\Delta_{\mathrm{B}}(-x)} \prod_{i=1}^{n} \frac{\left(t^{1 / 2} t_{2}^{-1} x_{i} ; t\right)_{r_{i}}}{\left(t^{1 / 2} t_{2} x_{i} ; t\right)_{r_{i}}}\left(t_{2} t^{1 / 2-n}\right)^{r_{i}}\left(x_{i}^{2} t^{r_{i}}\right)^{(m+2 n-1) r_{i} / 2},
\end{aligned}
$$

where $x_{n}:=1$. If we now let $t_{2}$ tend to zero, use that $h_{\lambda}^{(m)}(0 ; t)=1$ and further use [5, Lemmas $2.2 \&$ 2.4] (see also (5.34) for the former) to identify the right-hand sides as $\chi_{m}\left(\mathrm{~A}_{2 n}^{(2)}\right)$ and $\chi_{m}\left(\mathrm{D}_{n+1}^{(2)}\right)$, we obtain (5.7) and (5.16). We again note that the condition $x_{n}:=1$ in (5.30) implies that in the $\mathrm{D}_{n+1}^{(2)}$ case we must specialise $\mathrm{e}^{-\alpha_{n}} \mapsto-1$. Two further cases, already mentioned in relation with (5.27) and (5.28), arise from (5.29) and (5.30) by specialising $t_{2}=-t^{1 / 2}$. On the right we can once again use (5.26) as well as [5, Lemma 2.4] and Lemma A.3 to recognise the hypergeometric sums as

$$
\left(-t^{1 / 2} ; t^{1 / 2}\right)_{\infty} \chi_{m}(\mathfrak{g})
$$

for $\mathfrak{g}=\mathrm{D}_{n+1}^{(2)}$ and $\mathrm{B}_{n}^{(1) \dagger}$ respectively. In the latter case we must assume the specialisation $\mathrm{e}^{-\alpha_{n}} \mapsto \mathrm{e}^{-\alpha_{n-1}}$. On the left we use (2.10) and (2.11d) to find

$$
h_{\lambda}^{(m)}\left(t^{1 / 2} ; t\right)=\prod_{i=1}^{m-1}\left(-t^{1 / 2} ; t^{1 / 2}\right)_{m_{i}(\lambda)},
$$

completing the proofs of (5.11) and (5.18). To prove our final two results we consider (5.29) and (5.30) for $t_{2}=1$. Then, by (4.10), we can add the additional restriction " $\lambda$ ' is even" to the sum on the left and $|r| \equiv 0(\bmod 2)$ to the sum on the right. Using Lemmas A.2 and A.1 this proves (5.6) and (5.14).

\subsection{Rogers-Ramanujan identities.}

Starting with the pioneering papers [53 58], the link between affine Lie algebras and vertex operator algebras on the one hand and Rogers-Ramanujan identities on the other is by now well established, see also [2, 13, 14, 17, 18, 25, 26, 38, 71, 98]. Nonetheless, examples of $q$-series (as opposed to combinatorial) identities that lift classical Rogers-Ramanujan-type identities to affine Lie algebra at arbitrary rank and level while still permitting a product form, are rare. 
Recently Griffin et al. [31] showed how to use combinatorial character identities of the type proven in Section 5.1 to obtain doubly-infinite families of RogersRamanujan identities, including a generalisation of the Rogers-Ramanujan 82 and Andrews-Gordon [1,28, identities to the affine Lie algebra $\mathrm{A}_{2 n}^{(2)}$. Following the approach of [31] we prove several new doubly-infinite families of RogersRamanujan identities.

Theorem $5.10\left(\mathrm{~A}_{2 n}^{(2)}\right.$-Rogers-Ramanujan identities). Let $m, n$ be positive integers. Then

$$
\begin{aligned}
& \sum_{\substack{\lambda \\
\lambda_{1} \leqslant m}} q^{|\lambda| / 2} P_{\lambda}\left(1, q, q^{2}, \ldots ; q^{2 n}\right) \\
& \quad=\frac{\left(q^{\kappa} ; q^{\kappa}\right)_{\infty}^{n-1}\left(q^{\kappa / 2} ; q^{\kappa / 2}\right)_{\infty}}{(q ; q)_{\infty}^{n-1}\left(q^{1 / 2} ; q^{1 / 2}\right)_{\infty}} \prod_{i=1}^{n} \theta\left(q^{i} ; q^{\kappa / 2}\right) \prod_{1 \leqslant i<j \leqslant n} \theta\left(q^{j-i}, q^{i+j} ; q^{\kappa}\right)
\end{aligned}
$$

for $\kappa:=m+2 n+1$, and

$$
\begin{aligned}
& \sum_{\substack{\lambda \\
\lambda_{1} \leqslant 2 m}} t^{|\lambda| / 2+n \operatorname{odd}(\lambda)} P_{\lambda}\left(1, q, q^{2}, \ldots ; q^{2 n}\right) \\
& =\frac{\left(q^{\kappa} ; q^{\kappa}\right)_{\infty}^{n-1}\left(q^{\kappa / 2} ; q^{\kappa / 2}\right)_{\infty}}{(q ; q)_{\infty}^{n-1}\left(q^{1 / 2} ; q^{1 / 2}\right)_{\infty}} \prod_{i=1}^{n} \theta\left(q^{i+m} ; q^{\kappa / 2}\right) \prod_{1 \leqslant i<j \leqslant n} \theta\left(q^{j-i}, q^{i+j-1} ; q^{\kappa}\right)
\end{aligned}
$$

for $\kappa:=2 m+2 n+1$.

Proof. To prove (5.31) we apply the specialisation

$$
F: \mathbb{C}\left[\left[\mathrm{e}^{-\alpha_{0}}, \ldots, \mathrm{e}^{-\alpha_{n}}\right]\right] \rightarrow \mathbb{C}\left[\left[q^{1 / 2}\right]\right]
$$

given by

$$
F\left(\mathrm{e}^{-\alpha_{0}}\right)=q^{1 / 2} \text { and } F\left(\mathrm{e}^{-\alpha_{i}}\right)=q \quad \text { for } 1 \leqslant i \leqslant n
$$

to the $\mathrm{A}_{2 n}^{(2)}$ character identity (5.7). Since the the null root for $\mathrm{A}_{2 n}^{(2)}$ is given by $\delta=2 \alpha_{0}+\cdots+2 \alpha_{n-1}+\alpha_{n}$ it follows from (5.4) that

$$
F\left(x_{i}\right)=q^{n-i+1 / 2} \quad(1 \leqslant i \leqslant n) \quad \text { and } \quad F(t)=q^{2 n}
$$


Hence

$$
\begin{aligned}
F\left(\sum_{\substack{\lambda \\
\lambda_{1} \leqslant m}} t^{|\lambda| / 2} P_{\lambda}^{\prime}\left(x_{1}^{ \pm}, \ldots, x_{n}^{ \pm} ; t\right)\right) \\
=\sum_{\substack{\lambda \\
\lambda_{1} \leqslant m}} q^{n|\lambda|} P_{\lambda}^{\prime}\left(q^{-n+1 / 2}, q^{-n+3 / 2}, \ldots, q^{n-1 / 2} ; q^{2 n}\right) \\
=\sum_{\substack{\lambda \\
\lambda_{1} \leqslant m}} q^{|\lambda| / 2} P_{\lambda}^{\prime}\left(1, q, \ldots, q^{2 n-1} ; q^{2 n}\right) \\
=\sum_{\substack{\lambda \\
\lambda_{1} \leqslant m}} q^{|\lambda| / 2} P_{\lambda}\left(1, q, q^{2}, \ldots ; q^{2 n}\right) .
\end{aligned}
$$

Here the second equality uses the homogeneity of the modified Hall-Littlewood polynomial and the the third equality follows from (2.66) and

$$
f\left[\frac{1+q+\cdots+q^{2 n-1}}{1-q^{2 n}}\right]=f\left[1+q+q^{2}+\cdots\right], \quad f \in \Lambda .
$$

To apply $F$ to the left-hand side of (5.7) we use that for arbitrary $\Lambda \in P_{+}$ parametrised as (compare with (2.44b)

$$
\Lambda=c_{0} \Lambda_{0}+\left(\lambda_{1}-\lambda_{2}\right) \Lambda_{1}+\cdots+\left(\lambda_{n-1}-\lambda_{n}\right) \Lambda_{n-1}+\lambda_{n} \Lambda_{n}
$$

with $\lambda=\left(\lambda_{1}, \ldots, \lambda_{n}\right)$ a partition and $c_{0}$ a nonnegative integer we can rewrite the Weyl-Kac formula for $\mathrm{A}_{2 n}^{(2)}$ as [5, Lemma 2.2],

$$
\begin{aligned}
& \mathrm{e}^{-\Lambda} \operatorname{ch} V(\Lambda)=\frac{1}{(t ; t)_{\infty}^{n} \prod_{i=1}^{n} \theta\left(t^{1 / 2} x_{i} ; t\right) \theta\left(x_{i}^{2} ; t^{2}\right) \prod_{1 \leqslant i<j \leqslant n} x_{j} \theta\left(x_{i} / x_{j}, x_{i} x_{j} ; t\right)} \\
& \times \sum_{r \in \mathbb{Z}^{n}}{\widetilde{\mathrm{sp}_{2 n, \lambda}}}_{2}\left(x t^{r}\right) \prod_{i=1}^{n} x_{i}^{\kappa r_{i}+\lambda_{i}} t^{\kappa r_{i}^{2} / 2-n r_{i}} .
\end{aligned}
$$

Here $\kappa:=2 n+c_{0}+2 \lambda_{1}+1, \widetilde{\mathrm{sp}}_{2 n, \lambda}$ is the normalised symplectic Schur function (A.1) and $x_{1}, \ldots, x_{n}$ and $t$ are defined by (5.4). Using

$$
\begin{array}{r}
F\left((t ; t)_{\infty}^{n} \prod_{i=1}^{n} \theta\left(t^{1 / 2} x_{i} ; t\right) \theta\left(x_{i}^{2} ; t^{2}\right) \prod_{1 \leqslant i<j \leqslant n} x_{j} \theta\left(x_{i} / x_{j}, x_{i} x_{j} ; t\right)\right) \\
=(q ; q)_{\infty}^{n-1}\left(q^{1 / 2} ; q^{1 / 2}\right)_{\infty} q^{\sum_{i<j}(n-j+1 / 2)}
\end{array}
$$


as well as the equations (2.60), (A.1), and appealing to multilinearity, yields

$$
\begin{aligned}
F\left(\mathrm{e}^{-\Lambda} \operatorname{ch} V(\Lambda)\right) & =\frac{q^{-\sum_{i<j}(n-j+1 / 2)}}{(q ; q)_{\infty}^{n-1}\left(q^{1 / 2} ; q^{1 / 2}\right)_{\infty}} \\
\times \operatorname{det}_{1 \leqslant i, j \leqslant n}\left(\sum_{r \in \mathbb{Z}} q^{\left(\kappa r+\lambda_{i}-\lambda_{j}+j-1\right)(n-i+1 / 2)+n \kappa r^{2}-2 n r\left(\lambda_{j}+n-j+1\right)}\right. & \\
& \left.-\sum_{r \in \mathbb{Z}} q^{\left(\kappa r+\lambda_{i}+\lambda_{j}+2 n-j+1\right)(n-i+1 / 2)+n \kappa r^{2}+2 n r\left(\lambda_{j}+n-j+1\right)}\right) .
\end{aligned}
$$

Replacing $(i, j) \mapsto(n-j+1, n-i+1)$ in the determinant and then changing $r \mapsto-r-1$ in the second sum we get

$$
\begin{aligned}
& F\left(\mathrm{e}^{-\Lambda} \operatorname{ch} V(\Lambda)\right)=\frac{1}{(q ; q)_{\infty}^{n-1}\left(q^{1 / 2} ; q^{1 / 2}\right)_{\infty}} \\
& \quad \times \operatorname{det}_{1 \leqslant i, j \leqslant n}\left(\sum_{r \in \mathbb{Z}} x_{i}^{2 n r-i+1} q^{2 n \kappa\left(\begin{array}{c}
r \\
2
\end{array}\right)+\kappa r / 2}\left(\left(x_{i} q^{\kappa r}\right)^{j-1}-\left(x_{i} q^{\kappa r}\right)^{2 n-j}\right)\right),
\end{aligned}
$$

where $x_{i}:=q^{\kappa / 2-i-\lambda_{n-i+1}}$. Again using multilinearity and recalling the $\mathrm{B}_{n}$ Vandermonde determinant (2.63), this may be rewritten as

$$
\begin{aligned}
F\left(\mathrm{e}^{-\Lambda} \operatorname{ch} V(\Lambda)\right)=\frac{1}{(q ; q)_{\infty}^{n-1}\left(q^{1 / 2} ; q^{1 / 2}\right)_{\infty}} & \\
& \times \sum_{r \in \mathbb{Z}^{n}} \Delta_{\mathrm{B}}\left(x q^{\kappa r}\right) \prod_{i=1}^{n} x_{i}^{2 n r_{i}-i+1} q^{2 n \kappa\left(\begin{array}{c}
r_{i} \\
2
\end{array}\right)+\kappa r_{i} / 2} .
\end{aligned}
$$

By the $\mathrm{D}_{n+1}^{(2)}$ Macdonald identity [62] in the form given by [84, Corollary 6.2], i.e.,

$$
\begin{aligned}
\sum_{r \in \mathbb{Z}^{n}} \Delta_{\mathrm{B}}\left(x q^{r}\right) & \prod_{i=1}^{n} x_{i}^{2 n r_{i}-i+1} q^{2 n\left(\begin{array}{c}
r_{i} \\
2
\end{array}\right)+r_{i} / 2} \\
& =(q ; q)_{\infty}^{n-1}\left(q^{1 / 2} ; q^{1 / 2}\right)_{\infty} \prod_{i=1}^{n} \theta\left(x_{i} ; q^{1 / 2}\right) \prod_{1 \leqslant i<j \leqslant n} \theta\left(x_{i} / x_{j}, x_{i} x_{j} ; q\right),
\end{aligned}
$$

we obtain the product formula

$$
\begin{aligned}
F\left(\mathrm{e}^{-\Lambda} \operatorname{ch} V(\Lambda)\right)= & \frac{\left(q^{\kappa} ; q^{\kappa}\right)_{\infty}^{n-1}\left(q^{\kappa / 2} ; q^{\kappa / 2}\right)_{\infty}}{(q ; q)_{\infty}^{n-1}\left(q^{1 / 2} ; q^{1 / 2}\right)_{\infty}} \prod_{i=1}^{n} \theta\left(q^{\kappa / 2-i-\lambda_{n-i+1}} ; q^{\kappa / 2}\right) \\
& \times \prod_{1 \leqslant i<j \leqslant n} \theta\left(q^{\lambda_{n-j+1}-\lambda_{n-i+1}+j-i}, q^{\kappa-i-j-\lambda_{n-i+1}-\lambda_{n-j+1}} ; q^{\kappa}\right) .
\end{aligned}
$$


By $\theta(x ; q)=\theta(q / x ; q)$ and a reversal of the products, this simplifies to

$$
\begin{gathered}
F\left(\mathrm{e}^{-\Lambda} \operatorname{ch} V(\Lambda)\right)=\frac{\left(q^{\kappa} ; q^{\kappa}\right)_{\infty}^{n-1}\left(q^{\kappa / 2} ; q^{\kappa / 2}\right)_{\infty}}{(q ; q)_{\infty}^{n-1}\left(q^{1 / 2} ; q^{1 / 2}\right)_{\infty}} \prod_{i=1}^{n} \theta\left(q^{\lambda_{i}+n-i+1} ; q^{\kappa / 2}\right) \\
\times \prod_{1 \leqslant i<j \leqslant n} \theta\left(q^{\lambda_{i}-\lambda_{j}-i+j}, q^{\lambda_{i}+\lambda_{j}+2 n-i-j+2} ; q^{\kappa}\right) .
\end{gathered}
$$

For $\lambda=0$ and $c_{0}=m$ this gives the claimed right-hand side of (5.31).

To prove (5.32) we this time apply the specialisation

$$
F^{\dagger}: \mathbb{C}\left[\left[\mathrm{e}^{-\alpha_{0}}, \ldots, \mathrm{e}^{-\alpha_{n}}\right]\right] \rightarrow \mathbb{C}\left[\left[q^{1 / 2}\right]\right]
$$

given by

$$
F^{\dagger}\left(\mathrm{e}^{-\alpha_{n}}\right)=q^{1 / 2} \quad \text { and } \quad F^{\dagger}\left(\mathrm{e}^{-\alpha_{i}}\right)=q \quad \text { for } 0 \leqslant i \leqslant n-1
$$

in the $\mathrm{A}_{2 n}^{(2) \dagger}$ character identity (5.10). This implies the same specialisation of $x_{1}, \ldots, x_{n}$ and $q$ as before, i.e.,

$$
F^{\dagger}\left(x_{i}\right)=q^{n-i+1 / 2} \quad(1 \leqslant i \leqslant n) \quad \text { and } \quad F^{\dagger}(t)=q^{2 n}
$$

so that

$$
\begin{aligned}
F^{\dagger}\left(\sum_{\substack{\lambda \\
\lambda_{1} \leqslant 2 m}} t^{(|\lambda|+\operatorname{odd}(\lambda)) / 2} P_{\lambda}^{\prime}\left(x_{1}^{ \pm}, \ldots, x_{n}^{ \pm} ; t\right)\right) \\
=\sum_{\substack{\lambda \\
\lambda_{1} \leqslant 2 m}} q^{|\lambda| / 2+n \operatorname{odd}(\lambda)} P_{\lambda}\left(1, q, q^{2}, \ldots ; q^{2 n}\right) .
\end{aligned}
$$

Moreover, since (5.33) and (5.37) are compatible with the map from $\mathrm{A}_{2 n}^{(2)}$ to $\mathrm{A}_{2 n}^{(2) \dagger}$ (corresponding to a reversal of the labelling of simple roots) we can again use (5.36):

$$
\begin{aligned}
& \left.F^{\dagger}\left(\mathrm{e}^{-m \Lambda_{0}} \operatorname{ch} V\left(m \Lambda_{0}\right)\right)\right|_{\mathfrak{g}=\mathrm{A}_{2 n}^{(2) \dagger}} \\
& \quad=\left.F\left(\mathrm{e}^{-m \Lambda_{n}} \operatorname{ch} V\left(m \Lambda_{n}\right)\right)\right|_{\mathfrak{g}=\mathrm{A}_{2 n}^{(2)}} \\
& \quad=\frac{\left(q^{\kappa} ; q^{\kappa}\right)_{\infty}^{n-1}\left(q^{\kappa / 2} ; q^{\kappa / 2}\right)_{\infty}}{(q ; q)_{\infty}^{n-1}\left(q^{1 / 2} ; q^{1 / 2}\right)_{\infty}} \prod_{i=1}^{n} \theta\left(q^{i+m} ; q^{\kappa / 2}\right) \prod_{1 \leqslant i<j \leqslant n} \theta\left(q^{j-i}, q^{i+j-1} ; q^{\kappa}\right),
\end{aligned}
$$

where $\kappa=2 m+2 n+1$. 
Theorem $5.11\left(\mathrm{D}_{n+1}^{(2)}\right.$-Rogers-Ramanujan identities). For $m, n$ positive integers and $\kappa:=m+2 n$,

$$
\begin{aligned}
& \text { 5.38) } \sum_{\substack{\lambda \\
\lambda_{1} \leqslant m}} q^{|\lambda| / 2} P_{\lambda}\left(1, q, q^{2}, \ldots ; q^{2 n-1}\right) \\
& =\frac{\left(q^{\kappa} ; q^{\kappa}\right)_{\infty}^{n}}{(q ; q)_{\infty}^{n-1}\left(q^{1 / 2} ; q\right)_{\infty}\left(q^{2} ; q^{2}\right)_{\infty}} \prod_{i=1}^{n} \theta\left(q^{i+(m-1) / 2} ; q^{\kappa}\right) \prod_{1 \leqslant i<j \leqslant n} \theta\left(q^{j-i}, q^{i+j-1} ; q^{\kappa}\right),
\end{aligned}
$$

and

$$
\begin{aligned}
& \sum_{\substack{\lambda \\
\lambda_{1} \leqslant m}} q^{|\lambda| / 2}\left(\prod_{i=1}^{m-1}\left(-q^{n} ; q^{n}\right)_{m_{i}(\lambda)}\right) P_{\lambda}\left(1, q, q^{2}, \ldots ; q^{2 n}\right) \\
= & \frac{\left(q^{\kappa} ; q^{\kappa}\right)_{\infty}^{n-1}\left(q^{\kappa / 2} ; q^{\kappa / 2}\right)_{\infty}}{(q ; q)_{\infty}^{n-1}\left(q^{1 / 2} ; q\right)_{\infty}^{2}\left(q^{2} ; q^{2}\right)_{\infty}} \prod_{i=1}^{n} \theta\left(q^{i-1 / 2} ; q^{\kappa / 2}\right) \prod_{1 \leqslant i<j \leqslant n} \theta\left(q^{j-i}, q^{i+j-1} ; q^{\kappa}\right) .
\end{aligned}
$$

For $n=1$ the first of these identities is the second equation on page 235 of [94].

Sketch of proof. In the character identity (5.16) we carry out the specialisation

$$
F: \mathbb{C}\left[\left[\mathrm{e}^{-\alpha_{0}}, \ldots, \mathrm{e}^{-\alpha_{n}}\right]\right] \rightarrow \mathbb{C}\left[\left[q^{1 / 2}\right]\right]
$$

given by

$$
F\left(\mathrm{e}^{-\alpha_{0}}\right)=q^{1 / 2}, \quad F\left(\mathrm{e}^{-\alpha_{n}}\right)=-1 \quad \text { and } \quad F\left(\mathrm{e}^{-\alpha_{i}}\right)=q \quad \text { for } 1 \leqslant i \leqslant n-1 .
$$

Noting that $F$ applied to (5.15) yields

$$
F\left(x_{i}\right)=q^{n-i} \quad(1 \leqslant i \leqslant n-1), \quad F\left(t^{1 / 2}\right)=q^{n-1 / 2},
$$

and following the proof of (5.31), it follows that the right-hand side of (5.16) maps to the left-hand side of (5.38). If we parametrise $\Lambda \in P_{+}$as (compare with (2.44a $)$

$$
\Lambda=c_{0} \Lambda_{0}+\left(\lambda_{1}-\lambda_{2}\right) \Lambda_{1}+\cdots+\left(\lambda_{n-1}-\lambda_{n}\right) \Lambda_{n-1}+2 \lambda_{n} \Lambda_{n},
$$

with $\lambda=\left(\lambda_{1}, \ldots, \lambda_{n}\right)$ a partition or half-partition and $c_{0}$ a nonnegative, and again follow the previous proof, we find

$$
\begin{gathered}
F\left(\mathrm{e}^{-\Lambda} \operatorname{ch} V(\Lambda)\right)=\frac{\left(q^{\kappa} ; q^{\kappa}\right)_{\infty}^{n}}{(q ; q)_{\infty}^{n-1}\left(q^{1 / 2} ; q\right)_{\infty}\left(q^{2} ; q^{2}\right)_{\infty}} \prod_{i=1}^{n} \theta\left(q^{\lambda_{i}+n-i+(\kappa+1) / 2} ; q^{\kappa}\right) \\
\times \prod_{1 \leqslant i<j \leqslant n} \theta\left(q^{\lambda_{i}-\lambda_{j}-i+j}, q^{\lambda_{i}+\lambda_{j}+2 n-i-j+1} ; q^{\kappa}\right)
\end{gathered}
$$

where $\kappa:=2 n+c_{0}+2 \lambda_{1}$. The only change compared to the proof of (5.31) is that we have used the $\mathrm{B}_{n}^{(1)}$ instead of $\mathrm{D}_{n+1}^{(2)}$ Macdonald identity. For $\Lambda=m \Lambda_{0}$, 
i.e., $\lambda=0$ and $c_{0}=m$ the above product gives the right-hand side of (5.38), completing the proof.

Similarly, to prove (5.39) we apply the specialisation $F$ to (5.11), where this time

$$
\begin{gathered}
F: \mathbb{C}\left[\left[\mathrm{e}^{-\alpha_{0}}, \ldots, \mathrm{e}^{-\alpha_{n}}\right]\right] \rightarrow \mathbb{C}\left[\left[q^{1 / 2}\right]\right] \\
F\left(\mathrm{e}^{-\alpha_{0}}\right)=F\left(\mathrm{e}^{-\alpha_{n}}\right)=q^{1 / 2} \text { and } F\left(\mathrm{e}^{-\alpha_{i}}\right)=q \text { for } 1 \leqslant i \leqslant n-1 .
\end{gathered}
$$

Applied to (5.9) this gives

$$
F\left(x_{i}\right)=q^{n-i+1 / 2} \quad(1 \leqslant i \leqslant n), \quad F\left(t^{1 / 2}\right)=q^{n},
$$

so that the left side of (5.11) specialises to the the right side of (5.39). With the same parametrisation of $\Lambda$ as in (5.40) and once more using the $\mathrm{D}_{n+1}^{(2)}$ Macdonald identity, it follows that

$$
\begin{array}{r}
F\left(\mathrm{e}^{-\Lambda} \operatorname{ch} V(\Lambda)\right)=\frac{\left(q^{\kappa} ; q^{\kappa}\right)_{\infty}^{n-1}\left(q^{\kappa / 2} ; q^{\kappa / 2}\right)_{\infty}\left(-q^{n} ; q^{n}\right)_{\infty}}{(q ; q)_{\infty}^{n-1}\left(q^{1 / 2} ; q\right)_{\infty}^{2}\left(q^{2} ; q^{2}\right)_{\infty}} \prod_{i=1}^{n} \theta\left(q^{\lambda_{i}+n-i+1 / 2} ; q^{\kappa / 2}\right) \\
\times \prod_{1 \leqslant i<j \leqslant n} \theta\left(q^{\lambda_{i}-\lambda_{j}-i+j}, q^{\lambda_{i}+\lambda_{j}+2 n-i-j+1} ; q^{\kappa}\right)
\end{array}
$$

with $\kappa$ as before. For $\Lambda=m \Lambda_{0}$, i.e., $\lambda=0$ and $c_{0}=m$ this gives the right-hand side of (5.39).

Theorem 5.12 ( $\mathrm{B}_{n}^{(1)}$-Rogers-Ramanujan identity). Let $m, n$ be a positive integers and $\kappa:=m+2 n-1$. Then

$$
\begin{aligned}
& \sum_{\substack{\lambda \\
\lambda_{1} \leqslant m}} q^{|\lambda| / 2}\left(\prod_{i=1}^{m-1}\left(-q^{n-1 / 2} ; q^{n-1 / 2}\right)_{m_{i}(\lambda)}\right) P_{\lambda}\left(1, q, q^{2}, \ldots ; q^{2 n-1}\right) \\
= & \frac{\left(q^{\kappa} ; q^{\kappa}\right)_{\infty}^{n}}{(q ; q)_{\infty}^{n-1}\left(q^{1 / 2} ; q^{1 / 2}\right)_{\infty}} \prod_{i=1}^{n} \theta\left(q^{i+m / 2-1 / 2} ; q^{\kappa}\right) \prod_{1 \leqslant i<j \leqslant n} \theta\left(q^{j-i}, q^{i+j-2} ; q^{\kappa}\right) .
\end{aligned}
$$

It follows from

$$
P_{\lambda}\left(1, q, q^{2}, \ldots ; q\right)=\frac{q^{n(\lambda)}}{b_{\lambda}(q)} \stackrel{\lambda_{1} \leqq m}{=} \prod_{i=1}^{m} \frac{q^{\left(\begin{array}{c}
\lambda_{i}^{\prime} \\
2
\end{array}\right)}}{(q ; q)_{m_{i}(\lambda)}}
$$

that for $n=1$ (and after replacing $q \mapsto q^{2}, \lambda_{i}^{\prime} \mapsto N_{i}$ and $m_{i}(\lambda) \mapsto n_{i}$ ) the theorem simplifies to Bressoud's even modulus identity [9, 10]

$$
\sum_{n_{1} \geqslant \cdots \geqslant n_{m} \geqslant 0} \frac{q^{N_{1}^{2}+\cdots+N_{m}^{2}}}{(q ; q)_{n_{1}} \cdots(q ; q)_{n_{m-1}}\left(q^{2} ; q^{2}\right)_{n_{m}}}=\frac{\left(q^{m+1}, q^{m+1}, q^{2 m+2} ; q^{2 m+2}\right)_{\infty}}{(q ; q)_{\infty}},
$$


where $N_{i}=n_{i}+\cdots+n_{m}$. From this point of view it is perhaps more natural to express the sum-side of (5.41) in terms of the Hall-Littlewood polynomials $Q_{\lambda}(x ; t)=b_{\lambda}(t) P_{\lambda}(x ; t)$ :

$$
\sum_{\substack{\lambda \\ \lambda_{1} \leqslant m}} \frac{q^{|\lambda| / 2} Q_{\lambda}\left(1, q, q^{2}, \ldots ; p^{2}\right)}{b_{\lambda}(p)(-p ; p)_{\lambda_{m}^{\prime}}}, \quad p=q^{n-1 / 2} .
$$

Remark 5.13. If after the substitution (5.24) we let $x_{n}$ tend to $t^{1 / 2}$ instead of 1 , and then specialise $(a, b)=\left(-t^{1 / 2},-1\right)$, we obtain an identity for $\chi_{m}\left(\mathrm{~B}_{n}^{(1)}\right)$ not included in Section 5.1. Upon specialisation this yields a companion to (5.41) as follows:

$$
\begin{aligned}
\sum_{\substack{\lambda \\
\lambda_{1} \leqslant m}} q^{|\lambda|}\left(\prod_{i=1}^{m-1}\left(-q^{n-1 / 2} ; q^{n-1 / 2}\right)_{m_{i}(\lambda)}\right) P_{\lambda}\left(1, q, q^{2}, \ldots ; q^{2 n-1}\right) \\
\quad=\frac{\left(q^{\kappa} ; q^{\kappa}\right)_{\infty}^{n}}{(q ; q)_{\infty}^{n-1}\left(q^{1 / 2} ; q^{1 / 2}\right)_{\infty}} \prod_{i=1}^{n} \theta\left(q^{i-1 / 2} ; q^{\kappa}\right) \prod_{1 \leqslant i<j \leqslant n} \theta\left(q^{j-i}, q^{i+j-1} ; q^{\kappa}\right) .
\end{aligned}
$$

For $n=1$ this corresponds to [9, 10]

$$
\sum_{n_{1} \geqslant \cdots \geqslant n_{m} \geqslant 0} \frac{q^{N_{1}^{2}+\cdots+N_{m}^{2}+N_{1}+\cdots+N_{m}}}{(q ; q)_{n_{1}} \cdots(q ; q)_{n_{m-1}}\left(q^{2} ; q^{2}\right)_{n_{m}}}=\frac{\left(q, q^{2 m+1}, q^{2 m+2} ; q^{2 m+2}\right)_{\infty}}{(q ; q)_{\infty}} .
$$

Sketch of proof of Theorem 5.12. We start with the $\mathrm{B}_{n}^{(1) \dagger}$ formula (5.18) and apply

$$
\begin{gathered}
F^{\dagger}: \mathbb{C}\left[\left[\mathrm{e}^{-\alpha_{0}}, \ldots, \mathrm{e}^{-\alpha_{n}}\right]\right] \rightarrow \mathbb{C}\left[\left[q^{1 / 2}\right]\right] \\
F^{\dagger}\left(\mathrm{e}^{-\alpha_{0}}\right)=q^{1 / 2} \text { and } F^{\dagger}\left(\mathrm{e}^{-\alpha_{i}}\right)=q \quad \text { for } 1 \leqslant i \leqslant n .
\end{gathered}
$$

Applied to (5.17) this yields

$$
F^{\dagger}\left(x_{i}\right)=q^{n-i} \quad(1 \leqslant i \leqslant n-1), \quad F^{\dagger}(t)=q^{2 n-1},
$$

so that, up to a factor $\left(-q^{n-1 / 2} ; q^{n-1 / 2}\right)$, the left-hand side of (5.41) follows by application of $F^{\dagger}$.

To obtain the product-form on the right we first consider the more general $\mathrm{B}_{n}^{(1)}$ specialisation formula

$$
\begin{gathered}
F\left(\mathrm{e}^{-\Lambda} \operatorname{ch} V(\Lambda)\right)=\frac{\left(q^{\kappa} ; q^{\kappa}\right)_{\infty}^{n}\left(-q^{n-1 / 2} ; q^{n-1 / 2}\right)_{\infty}}{(q ; q)_{\infty}^{n-1}\left(q^{1 / 2} ; q^{1 / 2}\right)_{\infty}} \prod_{i=1}^{n} \theta\left(q^{\lambda_{i}+n-i+1 / 2} ; q^{\kappa}\right) \\
\times \prod_{1 \leqslant i<j \leqslant n} \theta\left(q^{\lambda_{i}-\lambda_{j}-i+j}, q^{\lambda_{i}+\lambda_{j}+2 n-i-j+1} ; q^{\kappa}\right)
\end{gathered}
$$


where $\Lambda$ and $\kappa$ are as in Lemma A.1, and where $F$ is the specialisation

$$
F\left(\mathrm{e}^{-\alpha_{n}}\right)=q^{1 / 2} \text { and } F\left(\mathrm{e}^{-\alpha_{i}}\right)=q \quad \text { for } 0 \leqslant i \leqslant n-1
$$

of $\mathrm{B}_{n}^{(1)}$. Proof of this result follows from the $\mathrm{B}_{n}^{(1)}$ Macdonald identity. Again $F^{\dagger}$ and $F$ are compatible so that

$$
\begin{aligned}
& \left.F^{\dagger}\left(\mathrm{e}^{-m \Lambda_{0}} \operatorname{ch} V\left(m \Lambda_{0}\right)\right)\right|_{\mathfrak{g}=\mathrm{B}_{n}^{(1) \dagger}} \\
& =\left.F\left(\mathrm{e}^{-m \Lambda_{n}} \operatorname{ch} V\left(m \Lambda_{n}\right)\right)\right|_{\mathfrak{g}=\mathrm{B}_{n}^{(1)}} \\
& =\frac{\left(q^{\kappa} ; q^{\kappa}\right)_{\infty}^{n}\left(-q^{n-1 / 2} ; q^{n-1 / 2}\right)_{\infty}}{(q ; q)_{\infty}^{n}\left(q^{1 / 2} ; q\right)_{\infty}} \theta\left(q^{i+m / 2-1 / 2} ; q^{\kappa}\right) \\
& \times \prod_{i=1} \theta\left(q^{j-i}, q^{i+j-2} ; q^{\kappa}\right),
\end{aligned}
$$

where $\kappa=m+2 n-1$. Up to the factor $\left(-q^{n-1 / 2} ; q^{n-1 / 2}\right)_{\infty}$ this is the right side of (5.41).

Theorem $5.14\left(\mathrm{~A}_{2 n-1}^{(2)}\right.$-Rogers-Ramanujan identities). Let $m, n$ be a positive integers and $\kappa:=2 m+2 n$. Then

$$
\begin{aligned}
& \sum_{\substack{\lambda \\
\lambda_{1} \leqslant 2 m}} q^{|\lambda| / 2+(n-1 / 2) \operatorname{odd}(\lambda)} P_{\lambda}\left(1, q, q^{2}, \ldots ; q^{2 n-1}\right) \\
& \quad=\frac{\left(q^{\kappa} ; q^{\kappa}\right)_{\infty}^{n}}{(q ; q)_{\infty}^{n}\left(q ; q^{2}\right)_{\infty}} \prod_{i=1}^{n} \theta\left(q^{i+\kappa / 2-1} ; q^{\kappa}\right) \prod_{1 \leqslant i<j \leqslant n} \theta\left(q^{j-i}, q^{i+j-2} ; q^{\kappa}\right)
\end{aligned}
$$

and

$$
\begin{aligned}
\sum_{\substack{\lambda \\
\lambda_{1} \leqslant 2 m}}^{\prime} q^{|\lambda| / 2+n \operatorname{odd}(\lambda)}\left(\prod_{i=1}^{2 m-1}\left(q^{2 n} ; q^{4 n}\right)_{\left\lceil m_{i}(\lambda) / 2\right\rceil}\right) P_{\lambda}\left(1, q, q^{2}, \ldots ; q^{2 n}\right) \\
=\frac{\left(q^{\kappa} ; q^{\kappa}\right)_{\infty}^{n}\left(-q^{\kappa / 2} ; q^{\kappa}\right)_{\infty}}{2(q ; q)_{\infty}^{n}} \\
\quad \times \prod_{i=1}^{n} \theta\left(-q^{i-1}, q^{i+\kappa / 2-1} ; q^{\kappa}\right) \prod_{1 \leqslant i<j \leqslant n} \theta\left(q^{j-i}, q^{i+j-2} ; q^{\kappa}\right),
\end{aligned}
$$

where the prime denotes the restriction $m_{i}(\lambda) \equiv 0(\bmod 2)$ for $i=1,3, \ldots, 2 m-$ 1.

In the rank-1 case (5.43) can also be written as

$$
\sum_{n_{1} \geqslant \cdots \geqslant n_{2 m} \geqslant 0} \frac{q^{\frac{1}{2}\left(N_{1}^{2}+\cdots+N_{2 m}^{2}+n_{1}+n_{3}+\cdots+n_{2 m-1}\right)}}{(q ; q)_{n_{1}} \cdots(q ; q)_{n_{2 m}}}=\frac{\left(q^{m+1}, q^{m+1}, q^{2 m+2} ; q^{2 m+2}\right)_{\infty}}{(q ; q)_{\infty}\left(q ; q^{2}\right)_{\infty}},
$$


where $N_{i}=n_{i}+\cdots+n_{2 m}$. Since [96, Lemma A.1]

$$
\begin{aligned}
\sum_{n_{1}, \ldots, n_{2 m} \geqslant 0} \frac{a_{1}^{n_{1}} a_{2}^{n_{2}} \cdots a_{2 m}^{n_{2 m}} q^{\frac{1}{2}\left(N_{1}^{2}+\cdots+N_{2 m}^{2}\right)}}{(q ; q)_{n_{1}} \cdots(q ; q)_{n_{2 m}}} & \\
& =\sum_{n_{1}, \ldots, n_{m} \geqslant 0} \frac{a_{2}^{n_{1}} a_{4}^{n_{2}} \cdots a_{2 m}^{n_{m}} q^{N_{1}^{2}+\cdots+N_{m}^{2}}\left(-q^{1 / 2-N_{1}} a_{1} / a_{2} ; q\right)_{N_{1}}}{(q ; q)_{n_{1}} \cdots(q ; q)_{n_{m}}}
\end{aligned}
$$

provided that $a_{2 i} / a_{2 i-1}=a_{2} / a_{1}$ for all $2 \leqslant i \leqslant m$, this may also be written as

$$
\sum_{n_{1}, \ldots, n_{m} \geqslant 0} \frac{q^{N_{1}^{2}+\cdots+N_{m}^{2}}\left(-q^{1-N_{1}} ; q\right)_{N_{1}}}{(q ; q)_{n_{1}} \cdots(q ; q)_{n_{m}}}=\frac{\left(q^{m+1}, q^{m+1}, q^{2 m+2} ; q^{2 m+2}\right)_{\infty}}{(q ; q)_{\infty}\left(q ; q^{2}\right)_{\infty}} .
$$

For $m=1$ this is identity (12) in Slater's list of Rogers-Ramanujan-type identities [87.

Sketch of proof. To first result follows from the principal specialisation [51,52, 60

$$
\begin{gathered}
F: \quad \mathbb{C}\left[\left[\mathrm{e}^{-\alpha_{0}}, \ldots, \mathrm{e}^{-\alpha_{n}}\right]\right] \rightarrow \mathbb{C}[[q]] \\
F\left(\mathrm{e}^{-\alpha_{i}}\right)=q \text { for } 0 \leqslant i \leqslant n
\end{gathered}
$$

applied to the $\mathrm{A}_{2 n-1}^{(2) \dagger}$ formula (5.19). Since the principal specialisation does not distinguish between $\mathrm{A}_{2 n-1}^{(2) \dagger}$ and $\mathrm{A}_{2 n-1}^{(2)}$, we can use the general $\mathrm{A}_{2 n-1}^{(2)}$ principal specialisation formula [52,60]

$$
\begin{aligned}
& F\left(\mathrm{e}^{-\Lambda} \operatorname{ch} V(\Lambda)\right)=\frac{\left(q^{\kappa} ; q^{\kappa}\right)_{\infty}^{n}}{(q ; q)_{\infty}^{n}\left(q ; q^{2}\right)_{\infty}} \prod_{i=1}^{n} \theta\left(q^{\lambda_{i}+n-i+1} ; q^{\kappa}\right) \\
& \times \prod_{1 \leqslant i<j \leqslant n} \theta\left(q^{\lambda_{i}-\lambda_{j}-i+j}, q^{\lambda_{i}+\lambda_{j}+2 n-i-j+2} ; q^{\kappa}\right)
\end{aligned}
$$

where $\kappa=2 n+c_{0}+\lambda_{1}+\lambda_{2}$ and

$$
\Lambda=c_{0} \Lambda_{0}+\left(\lambda_{1}-\lambda_{2}\right) \Lambda_{1}+\cdots+\left(\lambda_{n-1}-\lambda_{n}\right) \Lambda_{n-1}+\lambda_{n} \Lambda_{n}
$$

for $c_{0}$ is a nonnegative integer and $\lambda=\left(\lambda_{1}, \ldots, \lambda_{n}\right)$ a partition. Taking $c_{0}=0$ and $\lambda=m^{n}$ gives the right-hand side of (5.43). The left-hand side follows in the usual way, noting that

$$
F\left(x_{i}\right)=q^{n-i+1} \quad(1 \leqslant i \leqslant n-1) \text { and } F(t)=q^{2 n-1} .
$$

The identity (5.44) follows from the specialisation $F$ applied to the $\mathrm{A}_{2 n-1}^{(2) \dagger}$ formula (5.8), where now $F$ stands for

$$
\begin{gathered}
F: \mathbb{C}\left[\left[\mathrm{e}^{-\alpha_{0}}, \ldots, \mathrm{e}^{-\alpha_{n}}\right]\right] \rightarrow \mathbb{C}[[q]] \\
F\left(\mathrm{e}^{-\alpha_{i}}\right)=q \text { for } 0 \leqslant i \leqslant n-1 \text { and } F\left(\mathrm{e}^{-\alpha_{n}}\right)=q^{2} .
\end{gathered}
$$


According to (A.6) this yields

$$
F\left(x_{i}\right)=q^{n-i+1 / 2} \quad(1 \leqslant i \leqslant n) \quad \text { and } \quad F(t)=q^{2 n},
$$

which we should apply to the $\mathrm{A}_{2 n-1}^{(2) \dagger}$ character given in (A.7). Unlike the previous cases, the steps required to obtain the product form are slightly different to those in the proof of (5.31), and below we outline the main differences.

From (5.46), the $\mathrm{D}_{n}$ Vandermonde determinant and multilinearity, it follows that

$$
\begin{aligned}
F\left(2 \sum_{r \in \mathbb{Z}^{n}} \Delta_{\mathrm{D}}\left(x t^{r}\right) \prod_{i=1}^{n}(-1)^{r_{i}} x_{i}^{\kappa r_{i}-i+1} t^{\frac{1}{2} \kappa r_{i}^{2}-(n-1) r_{i}}\right) \\
=\operatorname{det}_{1 \leqslant i, j \leqslant n}\left(\sum_{r \in \mathbb{Z}}(-1)^{r} q^{(\kappa r-i+j)(n-i+1 / 2)+n \kappa r^{2}-2 n r(n-j)}\right. \\
\left.+\sum_{r \in \mathbb{Z}}(-1)^{r} q^{(\kappa r-i-j+2 n)(n-i+1 / 2)+n \kappa r^{2}+2 n r(n-j)}\right) .
\end{aligned}
$$

After interchanging $i$ and $j$ and negating $r$ in the first sum, the right-hand side becomes

$$
\begin{aligned}
& \operatorname{det}_{1 \leqslant i, j \leqslant n}\left(\sum_{r \in \mathbb{Z}}(-1)^{r} q^{2 n \kappa\left(\begin{array}{c}
r \\
2
\end{array}\right)+\kappa r / 2+(2 n r-i+1)(n-i)}\right. \\
& \times\left.\left(q^{(\kappa r+n-i)(j-1)}+q^{(\kappa r+n-i)(2 n-j)}\right)\right) \\
&=\operatorname{det}_{1 \leqslant i, j \leqslant n}\left(\sum_{r \in \mathbb{Z}}(-1)^{r} x_{i}^{2 n r-i+1} q^{2 n \kappa\left(\begin{array}{c}
r \\
2
\end{array}\right)+\kappa r / 2}\left(\left(x_{i} q^{\kappa r}\right)^{j-1}-\left(x_{i} q^{\kappa r}\right)^{2 n-j}\right)\right),
\end{aligned}
$$

where $x_{i}:=-q^{n-i}$. Up to the change $q^{\kappa / 2} \mapsto q^{-\kappa / 2}$, the above determinant is the same as the one on the right of (5.35). From here on we can thus follow the previous computations to find

$$
\begin{aligned}
F\left(2 \sum_{r \in \mathbb{Z}^{n}} \Delta_{\mathrm{D}}\left(x t^{r}\right) \prod_{i=1}^{n}(-1)^{r_{i}} x_{i}^{\kappa r_{i}-i+1} t^{\frac{1}{2} \kappa r_{i}^{2}-(n-1) r_{i}}\right) \\
=\left(q^{\kappa} ; q^{\kappa}\right)_{\infty}^{n}\left(-q^{\kappa / 2} ; q^{\kappa}\right)_{\infty} \prod_{i=1}^{n} \theta\left(-q^{i-1}, q^{i+\kappa / 2-1} ; q^{\kappa}\right) \\
\quad \times \prod_{1 \leqslant i<j \leqslant n} \theta\left(q^{j-i}, q^{i+j-2} ; q^{\kappa}\right) .
\end{aligned}
$$

We conclude this section with two remarks. First of all, we have not considered the specialisations of $(\underline{5.5})$ and $(\underline{5.13})$ as the resulting $\mathrm{C}_{n}^{(1)}$ and $\mathrm{A}_{2 n}^{(2)}$ 
identities were already obtained in [31], the $\mathrm{A}_{2 n}^{(2)}$ case corresponding to a generalisation of the Rogers-Ramanujan and Andrews-Gordon identities for odd moduli. We have also omitted the specialisation of (5.6) and (5.14), but for different reasons. The "right" substitutions on the combinatorial sides would be $x_{i} \mapsto q^{n-i+1 / 2},(1 \leqslant i \leqslant n), t \mapsto t^{2 n}$ and $x_{i} \mapsto q^{n-i+1}(1 \leqslant i \leqslant n-1)$, $t \mapsto t^{2 n-1}$ respectively. This corresponds to the specialisations

$$
F\left(\mathrm{e}^{-\alpha_{0}}\right)=q^{2} \quad \text { and } \quad F\left(\mathrm{e}^{-\alpha_{i}}\right)=q \quad \text { for } 1 \leqslant i \leqslant n
$$

for $\mathrm{A}_{2 n-1}^{(2)}$, and

$$
F\left(\mathrm{e}^{-\alpha_{i}}\right)=q \quad \text { for } 1 \leqslant i \leqslant n-1 \quad \text { and } \quad F\left(\mathrm{e}^{-\alpha_{0}}\right)=q^{2}, F\left(\mathrm{e}^{-\alpha_{n}}\right)=-1
$$

for $\mathrm{B}_{n}^{(2)}$. However, $F\left(\mathrm{e}^{-m \Lambda_{0}} \operatorname{ch} V\left(m \Lambda_{0}\right)\right)$ does not factor for such $F$.

\subsection{Kaneko-Macdonald hypergeometric series.}

Kaneko-Macdonald-type basic hypergeometric series are an important generalisation of ordinary basic hypergeometric series to multiple series with Macdonald polynomial argument. They have been extensively studied in the literature, see e.g., [4, 39, 40, 50, 67, 77, 78, 83, 95, 97, and are particularly useful for computing Selberg integrals and constant term identities.

For $x=\left(x_{1}, \ldots, x_{n}\right)$ the Kaneko-Macdonald basic hypergeometric series ${ }_{r+1} \Phi_{r}$ is defined as 39,67 ]

$$
{ }_{r+1} \Phi_{r}\left[\begin{array}{c}
a_{1}, \ldots, a_{r+1} \\
b_{1}, \ldots, b_{r}
\end{array} ; q, t ; x\right]:=\sum_{\lambda} \frac{\left(a_{1}, \ldots, a_{r+1} ; q, t\right)_{\lambda}}{\left(b_{1}, \ldots, b_{r} ; q, t\right)_{\lambda}} \cdot \frac{t^{n(\lambda)} P_{\lambda}(x ; q, t)}{C_{\lambda}^{-}(q ; q, t)} .
$$

For our purposes it suffices to consider the principal specialisation

$$
\begin{aligned}
{ }_{r+1} \Phi_{r}^{(n)}\left[\begin{array}{c}
a_{1}, \ldots, a_{r+1} \\
b_{1}, \ldots, b_{r}
\end{array} ;, t ; z\right] & :={ }_{r+1} \Phi_{r}\left[\begin{array}{c}
a_{1}, \ldots, a_{r+1} \\
b_{1}, \ldots, b_{r}
\end{array} q, t ; z\left(1, t, \ldots, t^{n-1}\right)\right] \\
& =\sum_{\substack{\lambda \\
l(\lambda) \leqslant n}} \frac{\left(t^{n}, a_{1}, \ldots, a_{r+1} ; q, t\right)_{\lambda}}{\left(b_{1}, \ldots, b_{r} ; q, t\right)_{\lambda}} \cdot \frac{z^{|\lambda|} t^{2 n(\lambda)}}{C_{\lambda}^{-}(q, t ; q, t)} .
\end{aligned}
$$

Since $C_{r}^{-}(q, t ; q, t)=(q, t ; q)_{r}$ we have ${ }_{r+1} \Phi_{r}^{(1)}={ }_{r+1} \phi_{r}$ with on the right an ordinary basic hypergeometric series, see [27]. Due to the factor $\left(t^{n} ; q, t\right)_{\lambda}$ the summand vanishes unless $l(\lambda) \leqslant n$. For generic $b_{1}, \ldots, b_{r}$ the restriction in the sum over $\lambda$ may thus be dropped. If $a_{r+1}=q^{-m}$ the series terminates with support given by $\lambda \subset m^{n}$. If

$$
t^{n-1} a_{1} \cdots a_{r+1}=b_{1} \cdots b_{r} \quad \text { and } \quad z=q
$$

we say that $\mathrm{a}_{r+1} \Phi_{r}^{(n)}$ is balanced. 
Replacing $\lambda$ by its complement with respect to $m^{n}$ and using (2.6) gives

$$
\begin{aligned}
{ }_{r+1} & \Phi_{r}^{(n)}\left[\begin{array}{c}
a_{1}, \ldots, a_{r}, q^{-m} \\
b_{1}, \ldots, b_{r}
\end{array} ; q, t ;\right] \\
= & \left(-\frac{z}{q}\right)^{m n} q^{-n\left(\begin{array}{c}
m \\
2
\end{array}\right)} t^{m\left(\begin{array}{c}
n \\
2
\end{array}\right)} \frac{\left(a_{1}, \ldots, a_{r} ; q, t\right)_{m^{n}}}{\left(b_{1}, \ldots, b_{r} ; q, t\right)_{m^{n}}} \\
& \times{ }_{r+1} \Phi_{r}^{(n)}\left[\begin{array}{c}
q^{1-m} t^{n-1} / b_{1}, \ldots, q^{1-m} t^{n-1} / b_{r}, q^{-m} \\
q^{1-m} t^{n-1} / a_{1}, \ldots, q^{1-m} t^{n-1} / a_{r}
\end{array} ; q, t ; \frac{b_{1} \cdots b_{r} q^{m+1}}{a_{1} \cdots a_{r} z t^{n-1}}\right] .
\end{aligned}
$$

Similarly, replacing $\lambda$ by its conjugate and applying (2.4) yields the duality

$$
\begin{aligned}
{ }_{r+1} \Phi_{r}^{(n)}\left[\begin{array}{r}
a_{1}, \ldots, a_{r}, q^{-m} \\
b_{1}, \ldots, b_{r}
\end{array} ; q, t ; z\right] \\
\quad={ }_{r+1} \Phi_{r}^{(m)}\left[\begin{array}{r}
1 / a_{1}, \ldots, 1 / a_{r}, t^{-n} \\
1 / b_{1}, \ldots, 1 / b_{r}
\end{array} ; t, q ; \frac{a_{1} \cdots a_{r} z t^{n}}{b_{1} \cdots b_{r} q^{m}}\right]
\end{aligned}
$$

The expression of the (monic) Askey-Wilson polynomials as a balanced ${ }_{4} \phi_{3}$ series [3] has a perfect analogue for the Koornwinder polynomials indexed by $m^{n}$.

Lemma 5.15. For $m$ a nonnegative integer

$$
\begin{aligned}
& K_{m^{n}}\left(z\left(1, t, \ldots, t^{n-1}\right) ; q, t ; t_{0}, t_{1}, t_{2}, t_{3}\right) \\
&=t_{0}^{-m n} t^{-m\left(\begin{array}{c}
n \\
2
\end{array}\right)} \frac{\left(t_{0} t_{1} t^{n-1}, t_{0} t_{2} t^{n-1}, t_{0} t_{3} t^{n-1} ; q, t\right)_{m^{n}}}{\left(t_{0} t_{1} t_{2} t_{3} q^{m-1} t^{n-1} ; q, t\right)_{m^{n}}} \\
& \quad \times{ }_{4} \Phi_{3}^{(n)}\left[\begin{array}{c}
z t_{0} t^{n-1}, t_{0} / z, t_{0} t_{1} t_{2} t_{3} q^{m-1} t^{n-1}, q^{-m} \\
t_{0} t_{1} t^{n-1}, t_{0} t_{2} t^{n-1}, t_{0} t_{3} t^{n-1}
\end{array} ;, t ; q\right] .
\end{aligned}
$$

For later use we note that by (5.47) and

$$
(a ; q, t)_{m^{n}}=(-a)^{m n} q^{n\left(\begin{array}{c}
m \\
2
\end{array}\right)} t^{-m\left(\begin{array}{c}
n \\
2
\end{array}\right)}\left(q^{1-m} t^{n-1} / a ; q, t\right)_{m^{n}}
$$

we may rewrite (5.49) as

$$
\begin{aligned}
& K_{m^{n}}\left(z\left(1, t, \ldots, t^{n-1}\right) ; q, t ; t_{0}, t_{1}, t_{2}, t_{3}\right) \\
&=z^{-m n} t^{-m\left(\begin{array}{c}
n \\
2
\end{array}\right)}\left(z t_{0} t^{n-1}, z q^{1-m} t^{n-1} / t_{0} ; q, t\right)_{m^{n}} \\
& \quad \times{ }_{4} \Phi_{3}^{(n)}\left[\begin{array}{c}
q^{1-m} / t_{0} t_{1}, q^{1-m} / t_{0} t_{2}, q^{1-m} / t_{0} t_{3}, q^{-m} \\
z q^{1-m} t^{n-1} / t_{0}, q^{1-m} / z t_{0}, q^{2-2 m} / t_{0} t_{1} t_{2} t_{3}
\end{array} ; q, t ; q\right] .
\end{aligned}
$$

Proof of Lemma 5.15. Okounkov's binomial formula [73, Theorem 7.10] gives an expansion of the Koornwinder polynomials in terms of $\mathrm{BC}_{n}$ interpolation polynomials $\bar{P}_{\mu}^{*}(x ; q, t, s)$ [73,77]. The coefficients in this expansion are given by $\mathrm{BC}_{n} q$-binomial coefficients $\left[\begin{array}{l}\lambda \\ \mu\end{array}\right]_{q, t, s}$ (see e.g., [77, page 64$]$ ) times a ratio of 
principally specialised Koornwinder polynomials:

$$
\begin{aligned}
K_{\lambda}(x ; q, t ; & \left.t_{0}, t_{1}, t_{2}, t_{3}\right) \\
& =\sum_{\mu \subset \lambda}\left[\begin{array}{l}
\lambda \\
\mu
\end{array}\right]_{q, t, s} \frac{K_{\lambda}\left(t_{0}\left(1, t, \ldots, t^{n-1}\right) ; q, t ; t_{0}, t_{1}, t_{2}, t_{3}\right)}{K_{\mu}\left(t_{0}\left(1, t, \ldots, t^{n-1}\right) ; q, t ; t_{0}, t_{1}, t_{2}, t_{3}\right)} \bar{P}_{\mu}^{*}\left(x ; q, t, t_{0}\right),
\end{aligned}
$$

where $s=t^{n-1} \sqrt{t_{0} t_{1} t_{2} t_{3} / q}$.

Now let 77 .

$$
C_{\lambda}^{+}(z ; q, t):=\prod_{(i, j) \in \lambda}\left(1-z q^{\lambda_{i}+j-1} t^{2-\lambda_{j}^{\prime}-i}\right) .
$$

By [77, Proposition 4.1]

$$
\left[\begin{array}{c}
m^{n} \\
\mu
\end{array}\right]_{q, t, s}=(-q)^{|\mu|} t^{n(\mu)} q^{n\left(\mu^{\prime}\right)} \frac{\left(t^{n}, q^{-m}, s^{2} q^{m} t^{1-n} ; q, t\right)_{\mu}}{C_{\mu}^{-}(q, t ; q, t) C_{\mu}^{+}\left(s^{2} ; q, t\right)}
$$

and the specialisation formulas $[23,85]$

$$
\begin{aligned}
K_{\lambda}\left(t_{0}\left(1, t, \ldots, t^{n-1}\right) ; q, t\right. & \left.; t_{0}, t_{1}, t_{2}, t_{3}\right) \\
= & \frac{t^{n(\lambda)}}{\left(t_{0} t^{n-1}\right)^{|\lambda|}} \cdot \frac{\left(t^{n}, t_{0} t_{1} t^{n-1}, t_{0} t_{2} t^{n-1}, t_{0} t_{3} t^{n-1} ; q, t\right)_{\lambda}}{C_{\lambda}^{-}(t ; q, t) C_{\lambda}^{+}\left(t_{0} t_{1} t_{2} t_{3} t^{2 n-2} / q ; q, t\right)}
\end{aligned}
$$

and [77, Corollary 3.11]

$$
\bar{P}_{\mu}^{*}\left(z\left(1, t, \ldots, t^{n-1}\right) ; q, t, s\right)=\frac{t^{2 n(\mu)} q^{-n\left(\mu^{\prime}\right)}}{\left(-s t^{n-1}\right)^{|\mu|}} \cdot \frac{\left(t^{n}, s / z, s z t^{n-1} ; q, t\right)_{\mu}}{C_{\mu}^{-}(t ; q, t)}
$$

we obtain (5.51).

Lemma 2.3 implies an analogue of (5.51) for the Macdonald-Koornwinder polynomial $K_{m^{n}}\left(q, t ; t_{2}, t_{3}\right)$.

Lemma 5.16. For $m$ an integer or half-integer

$$
\begin{aligned}
& K_{m^{n}}\left(z\left(1, t, \ldots, t^{n-1}\right) ; q, t ; t_{2}, t_{3}\right) \\
& =z^{-m n} t^{-m\left(\begin{array}{c}
n \\
2
\end{array}\right)\left(-z q^{1 / 2-m} t^{n-1} ; q, t\right)_{(2 m)^{n}}} \\
& \quad \times{ }_{4} \Phi_{3}^{(n)}\left[\begin{array}{c}
-q^{1 / 2-m} / t_{2},-q^{1 / 2-m} / t_{3}, q^{1 / 2-m}, q^{-m} \\
-z q^{1 / 2-m} t^{n-1},-q^{1 / 2-m} / z, q^{3 / 2-2 m} / t_{2} t_{3}
\end{array} ; q, t ; q\right] .
\end{aligned}
$$

Proof. In the integer case (5.52) follows immediately from (5.51) by specialising $\left\{t_{0}, t_{1}\right\}=\left\{-q^{1 / 2},-1\right\}$ and applying

$$
\left(a, a q^{-m} ; q, t\right)_{m^{n}}=\left(a q^{-m} ; q, t\right)_{(2 m)^{n}}
$$


By Lemma 2.3, for $m=k+1 / 2$ a half-integer we need to show that

$$
\begin{aligned}
& K_{k^{n}}\left(z\left(1, t, \ldots, t^{n-1}\right) ; q, t ;-q,-q^{1 / 2}, t_{2}, t_{3}\right) \\
& =z^{-k n} t^{-k\left(\begin{array}{c}
n \\
2
\end{array}\right)}\left(-z q^{-k} t^{n-1},-z q t^{n-1} ; q, t\right)_{k^{n}} \\
& \quad \times{ }_{4} \Phi_{3}^{(n)}\left[\begin{array}{c}
-q^{-k} / t_{2}, q^{-k} / t_{3}, q^{-k-1 / 2}, q^{-k} \\
-z q^{-k} t^{n-1},-q^{-k} / z, q^{1 / 2-2 k} / t_{2} t_{3}
\end{array} ; q, t ; q\right],
\end{aligned}
$$

where we have also used

$$
\left.\prod_{i=1}^{n}\left(x_{i}^{1 / 2}+x_{i}^{-1 / 2}\right)\right|_{x_{i}=z t^{i-1}}=z^{-n / 2} t^{-\left(\begin{array}{c}
n \\
2
\end{array}\right) / 2}(-z ; t)_{n}
$$

and

$$
\frac{\left(-z q^{-k} t^{n-1} ; q, t\right)_{(2 k+1)^{n}}}{(-z ; t)_{n}}=\left(-z q^{-k} t^{n-1}, z q t^{n-1} ; q, t\right)_{k^{n}} .
$$

Since (5.54) is (5.51) with $\left(t_{0}, t_{1}, m\right) \mapsto\left(-q,-q^{1 / 2}, k\right)$ we are done.

Equipped with the above lemmas we can specialise Theorems 4.1 and 4.6 to obtain new transformation formulas for Kaneko-Macdonald-type hypergeometric series.

Proposition 5.17. For $m$ a nonnegative integer

$$
\begin{aligned}
& { }_{4} \Phi_{3}^{(n)}\left[\begin{array}{c}
a,-a,-t^{n}, q^{-m} \\
a q^{1 / 2} t^{n},-a q^{1 / 2} t^{n},-q^{-m} / t
\end{array} ; q, t ; q\right] \\
& =\frac{\left(a^{2} t\right)^{m n} q^{m^{2} n}\left(q t^{2 n} ; q, t\right)_{m^{n}}}{\left(a^{2} q t^{2 n} ; q^{2}, t^{2}\right)_{m^{n}}\left(-q t^{n} ; q, t\right)_{m^{n}}} \Phi_{1}^{(n)}\left[\begin{array}{r}
q^{-m} / t, q^{-m} \\
q t^{2 n-1}
\end{array} ; q, t^{2} ; \frac{q}{a^{2}}\right] .
\end{aligned}
$$

Interestingly, even the $n=1$ case of this transformation, given by

$$
\begin{aligned}
& { }_{4} \phi_{3}\left[\begin{array}{c}
a,-a, b, q^{-m} \\
a b q^{1 / 2},-a b q^{1 / 2}, q^{-m} / b
\end{array} ; q, q\right] \\
& =\frac{\left(-a^{2} b\right)^{m} q^{m^{2}}\left(b^{2} q ; q\right)_{m}}{\left(a^{2} b^{2} q ; q^{2}\right)_{m}(b q ; q)_{m}}{ }_{2} \phi_{1}\left[\begin{array}{c}
-q^{-m} / b, q^{-m} \\
-b q
\end{array} ; q, \frac{q}{a^{2}}\right],
\end{aligned}
$$

appears to be new.

Proof. In (4.5) we specialise $x \mapsto z\left(1, t, \ldots, t^{n-1}\right)$ and replace the summation index $\lambda$ by $2 \lambda$. On the left side we then use

$$
b_{2 \lambda ; m}^{\mathrm{oa}}(q, t)=\left(\frac{q}{t}\right)^{|\lambda|} \frac{\left(q^{-2 m} ; q^{2}, t\right)_{\lambda}}{\left(q^{1-2 m} / t ; q^{2}, t\right)_{\lambda}} \cdot \frac{C_{\lambda}^{-}\left(q t ; q^{2}, t\right)}{C_{\lambda}^{-}\left(q^{2} ; q^{2}, t\right)}
$$

(see the proof of Theorem 4.1 on page 45) and

$$
P_{2 \lambda}\left(z\left(1, t, \ldots, t^{n-1}\right) ; q, t\right) \stackrel{(2.30)}{=} z^{2|\lambda|} t^{2 n(\lambda)} \frac{\left(t^{n} ; q, t\right)_{2 \lambda}}{C_{2 \lambda}^{-}(t ; q, t)}=z^{2|\lambda|} t^{2 n(\lambda)} \frac{\left(t^{n}, q t^{n} ; q^{2}, t\right)_{\lambda}}{C_{\lambda}^{-}\left(t, q t ; q^{2}, t\right)} .
$$


This yields the ${ }_{2} \Phi_{1}^{(n)}$ series

$$
{ }_{2} \Phi_{1}^{(n)}\left[\begin{array}{c}
q t^{n}, q^{-2 m} \\
q^{1-2 m} / t
\end{array} ; q^{2}, t ; \frac{z^{2} q}{t}\right] .
$$

On the right we use (5.51) with

$$
\left(t_{0}, t_{1}, t_{2}, t_{3}\right)=\left(q^{1 / 2},-q^{1 / 2},(q t)^{1 / 2},-(q t)^{1 / 2}\right)
$$

and (5.53) to find

$$
\left(z q^{1 / 2-m} t^{n-1} ; q, t\right)_{(2 m)^{n}{ }_{4} \Phi_{3}^{(n)}}\left[\begin{array}{c}
q^{-m} t^{-1 / 2},-q^{-m} t^{-1 / 2},-q^{-m}, q^{-m} \\
z q^{1 / 2-m} t^{n-1}, q^{1 / 2-m} / z, q^{-2 m} t^{-1}
\end{array} ; q, t ; q\right] .
$$

We now apply the duality (5.48) to both sides and interchange $m$ and $n$ as well as $q$ and $t$. By

$$
(a ; t, q)_{n^{m}}=\left(a q^{1-m} t^{n-1} ; q, t\right)_{m^{n}}
$$

(which follows from (2.4a) and (2.6a) for $\lambda=m^{n}$ ) this yields

$$
\begin{aligned}
{ }_{2} \Phi_{1}^{(n)}\left[\begin{array}{r}
q^{-m} / t, q^{-m} \\
q t^{2 n-1}
\end{array}\right. & \left.; q, t^{2} ; z^{2} q^{2 m} t\right] \\
& =\left(z t^{n-1 / 2} ; q, t\right)_{m^{2 n}{ }_{4}} \Phi_{3}^{(n)}\left[\begin{array}{c}
q^{1 / 2} t^{n},-q^{1 / 2} t^{n},-t^{n}, q^{-m} \\
z t^{n-1 / 2}, q^{1-m} t^{n-1 / 2} / z, q t^{2 n}
\end{array} ;, t ; q\right] .
\end{aligned}
$$

Next we rewrite the right-hand side using the multiple Sears transformation 4 , Eq. (5.10)]

$$
\begin{aligned}
& { }_{4} \Phi_{3}^{(n)}\left[\begin{array}{c}
a, b, c, q^{-m} \\
d, e, q^{1-m} t^{n-1} a b c / d e
\end{array} ; q, t ; q\right]
\end{aligned}
$$

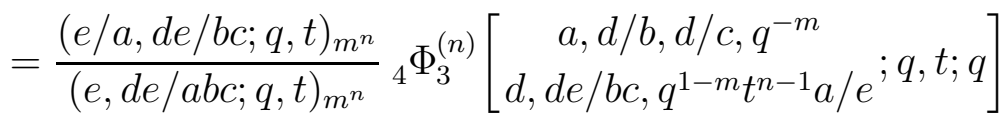

with

$$
(a, b, c, d, e) \mapsto\left(-t^{n}, q^{1 / 2} t^{n},-q^{1 / 2} t^{n}, z t^{n-1 / 2}, q^{1-m} t^{n-1 / 2} / z\right),
$$

and use

$$
\begin{aligned}
& \left(z t^{n-1 / 2} ; q, t\right)_{m^{2 n}} \frac{\left(-z t^{-1 / 2},-q^{-m} / t ; q, t\right)_{m^{n}}}{\left(z t^{n-1 / 2}, q^{-m} t^{-1-n} ; q, t\right)_{m^{n}}} \\
& =\frac{\left(z^{2} t^{-1} ; q^{2}, t^{2}\right)_{m^{n}}\left(-q^{-m} t^{-1} ; q, t\right)_{m^{n}}}{\left(q^{-m} t^{-1-n} ; q, t\right)_{m^{n}}}
\end{aligned}
$$

to clean up the prefactor. By the substitution $z \mapsto q^{1 / 2-m} t^{-1 / 2} / a$ and application of (5.50) the claim follows. 
Proposition 5.18. For $m$ a nonnegative integer

$$
\begin{aligned}
& { }_{4} \Phi_{3}^{(n)}\left[\begin{array}{c}
a, a q, q^{-m}, q^{1-m} \\
a q^{1-m} / t, a q^{2-m} / t, q t^{2 n}
\end{array} ; q^{2}, t^{2} ; q^{2}\right] \\
& =\frac{\left(t^{2 n} ; q^{2}, t^{2}\right)_{m^{n}}}{\left(t^{2 n}, t^{2 n-1} / a ; q, t^{2}\right)_{m^{n}}}{ }_{2} \Phi_{1}^{(n)}\left[\begin{array}{l}
-t^{n}, q^{-m} \\
-q^{1-m} / t
\end{array} ; q, t ; \frac{q}{a}\right] .
\end{aligned}
$$

For $n=1$, and up to the change $t \mapsto b$, this is a transformation stated on page 2310 of [6]. By iterating the Sears transformation (5.55) we obtain

$$
\begin{aligned}
& { }_{4} \Phi_{3}^{(n)}\left[\begin{array}{c}
a, b, c, q^{-m} \\
d, e, q^{1-m} t^{n-1} a b c / d e
\end{array} ; q, t ; q\right] \\
& =\frac{(a, e f / a b, e f / a c ; q, t)_{m^{n}}}{(e, f, e f / a b c ; q, t)_{m^{n}}} \Phi_{3}^{(n)}\left[\begin{array}{c}
e / a, f / a, e f / a b c, q^{-m} \\
e f / a b, e f / a c, q^{1-m} t^{n-1} / a
\end{array} ; q, t ; q\right],
\end{aligned}
$$

where $f:=q^{1-m} t^{n-1} a b c / d e$. If we let $b$ tend to zero and $c, d \rightarrow \infty$ such that $c / d \rightarrow z / q$, we find

$$
\begin{aligned}
& { }_{2} \Phi_{1}^{(n)}\left[\begin{array}{r}
a, q^{-m} \\
e^{-m}
\end{array}\right. \\
& \quad=\frac{(a, t ; z}{\left(a, z q^{1-m} t^{n-1} ; q, t\right)_{m^{n}}}{ }_{3} \Phi_{2}^{(n)}\left[\begin{array}{c}
0, e / a, q^{-m} \\
z q^{-m} t^{n-1}, q^{1-m} t^{n-1} / a
\end{array} ; q, t ; q\right] .
\end{aligned}
$$

This can be used to transform the right-hand side of (5.56) so that an equivalent form of that identity is given by

$$
\begin{aligned}
& { }_{4} \Phi_{3}^{(n)}\left[\begin{array}{c}
a, a q, q^{-m}, q^{1-m} \\
a q^{1-m} / t, a q^{2-m} / t, q t^{2 n}
\end{array} ; q^{2}, t^{2} ; q^{2}\right] \\
& =q^{-n\left(\begin{array}{c}
m \\
2
\end{array}\right)} \frac{(a ; q, t)_{m^{n}}\left(t^{2 n} ; q^{2}, t^{2}\right)_{m^{n}}{ }_{3} \Phi_{2}^{(n)}}{\left(a q^{1-m} / t, t^{2 n} ; q, t^{2}\right)_{m^{n}}}\left[\begin{array}{c}
0, q^{1-m} / t^{n+1}, q^{-m} \\
-q^{1-m} / t, q^{1-m} t^{n-1} / a
\end{array} ; q, t ; q\right] .
\end{aligned}
$$

This generalises the $c=a q^{1-m} / t$ case of Jain's quadratic transformation [36, Equation (3.6)]:

$$
\begin{aligned}
{ }_{4} \phi_{3}\left[\begin{array}{r}
a, a q, q^{-m}, q^{1-m} \\
c, c q, q t^{2}
\end{array} q^{2}, q^{2}\right] \\
\quad=q^{-\left(\begin{array}{c}
m \\
2
\end{array}\right)} \frac{(a ; q)_{m}\left(t^{2} ; q^{2}\right)_{m}}{\left(c, t^{2} ; q\right)_{m}}{ }_{4} \phi_{3}\left[\begin{array}{c}
0, c / a, q^{1-m} / t^{2}, q^{-m} \\
q^{1-m} / t,-q^{1-m} / t, q^{1-m} / a
\end{array} ; q, q\right] .
\end{aligned}
$$

Proof of Proposition 5.18. This time we specialise $x=z\left(1, t^{2}, \ldots, t^{2 n-2}\right)$ in (4.12). On the left we use (2.30) and

$$
b_{\lambda ; m}^{-}(q, t)=\left(-\frac{q}{t}\right)^{|\lambda|} \frac{\left(q^{-m} ; q, t\right)_{\lambda}}{\left(-q^{1-m} / t ; q, t\right)_{\lambda}} \cdot \frac{C_{\lambda}^{-}(-t ; q, t)}{C_{\lambda}^{-}(q ; q, t)}
$$


(see the proof of Theorem 4.6 on page 48), as well as

$$
\left(a^{2} ; q^{2}, t^{2}\right)_{\lambda}=(a,-a ; q, t)_{\lambda} \quad \text { and } \quad C_{\lambda}\left(a^{2} ; q^{2}, t^{2}\right)=C_{\lambda}(a,-a ; q, t) .
$$

On the right we first write

$$
P_{\left(\frac{m}{2}\right)^{n}}^{\left(\mathrm{B}_{n}, \mathrm{C}_{n}\right)}\left(x ; q^{2}, t^{2},-t\right)=K_{\left(\frac{m}{2}\right)^{n}}\left(x ; q^{2}, t^{2} ;-t,-q t\right)
$$

using (2.50b), then specialise $x$ and finally apply (5.52). As a result,

$$
\begin{aligned}
{ }_{2} \Phi_{1}^{(n)}\left[\begin{array}{l}
-t^{n}, q^{-m} \\
-q^{1-m} / t
\end{array} ; q, t ;-\frac{z q}{t}\right] & =\left(-z q^{1-m} t^{2 n-2} ; q^{2}, t^{2}\right)_{m^{n}} \\
& \times{ }_{4} \Phi_{3}^{(n)}\left[\begin{array}{c}
q^{-m} / t, q^{1-m} / t, q^{-m}, q^{1-m} \\
-z q^{1-m} t^{2 n-2},-q^{1-m} / z, q^{2-m} / t^{2}
\end{array} ; q^{2}, t^{2} ; q^{2}\right] .
\end{aligned}
$$

Again we apply the Sears transformation (5.55), this time with

$$
\begin{aligned}
& (a, b, c, d, e, m, q, t) \\
& \quad \mapsto\left(q^{1-2\lceil m / 2\rceil}, q^{-m} / t, q^{1-m} / t,-q^{1-m} / z,-z q^{1-m} t^{2 n-2},\lfloor m / 2\rfloor, q^{2}, t^{2}\right) .
\end{aligned}
$$

Noting that

$$
\begin{aligned}
\left(-z q^{1-m} t^{2 n-2} ; q^{2}, t^{2}\right)_{m^{n}} & =\frac{\left(-z q^{-m+2\lceil m / 2\rceil} t^{2 n-2}, q t^{2 n} ; q^{2}, t^{2}\right)_{\lfloor m / 2\rfloor^{n}}}{\left(-z q^{1-m} t^{2 n-2}, q^{2\lceil m / 2\rceil} t^{2 n} ; q^{2}, t^{2}\right)_{\lfloor m / 2\rfloor^{n}}} \\
& =\frac{\left(t^{2 n},-z t^{2 n-2} ; q, t^{2}\right)_{m^{n}}}{\left(t^{2 n} ; q^{2}, t^{2}\right)_{m^{n}}}
\end{aligned}
$$

and replacing $z \mapsto-t / a$ completes the proof.

\section{Open PROBLEMS}

We conclude this paper with a list of open problems.

If we specialise $\left\{t_{2}, t_{3}\right\}=\left\{ \pm t^{1 / 2}\right\}$ or $\left\{t_{2}, t_{3}\right\}=\left\{ \pm \mathrm{i} t^{1 / 4}\right\}$ in Theorem 4.7then the Rogers-Szegö polynomials in the summand factorise by (2.11).

Open problem 1. Find q-analogues of

$$
\sum t^{\operatorname{odd}(\lambda) / 2}\left(\prod_{i=1}^{2 m-1}\left(t ; t^{2}\right)_{\left\lceil m_{i}(\lambda) / 2\right\rceil}\right) P_{\lambda}(x ; t)=\left(x_{1} \cdots x_{n}\right)^{m} P_{m^{n}}^{\left(\mathrm{C}_{n}\right)}(x ; t, t)
$$


and

$$
\begin{aligned}
\sum t^{\operatorname{odd}(\lambda) / 4} & \left(\prod_{\substack{i=1 \\
i \text { even }}}^{2 m-1}\left(t^{1 / 2} ; t\right)_{\left\lceil m_{i}(\lambda) / 2\right\rceil}(-t ; t)_{\left\lfloor m_{i}(\lambda) / 2\right\rfloor}\right) \\
& \times\left(\prod_{i=1}^{2 m-1}\left(t ; t^{2}\right)_{m_{i}(\lambda) / 2}\right) P_{\lambda}(x ; t)=\left(x_{1} \cdots x_{n}\right)^{m} P_{m^{n}}^{\left(\mathrm{C}_{n}\right)}\left(x ; t, t^{1 / 2}\right) .
\end{aligned}
$$

In both cases the sum is over partitions $\lambda$ such that $\lambda_{1} \leqslant 2 m$ and $m_{i}(\lambda)$ is even for $i=1,3, \ldots, 2 m-1$.

Similarly, if we specialise $t_{2}=0$ in Theorem 4.8 then the Rogers-Szegö polynomial in the summand trivialises to 1.

Open problem 2. Find a q-analogue of

$$
\sum_{\substack{\lambda \\ \lambda_{1} \leqslant m}} P_{\lambda}(x ; t)=\left(x_{1} \cdots x_{n}\right)^{\frac{m}{2}} P_{\left(\frac{m}{2}\right)^{n}}^{\left(\mathrm{B}_{n}\right)}(x ; t, 0) .
$$

The bounded Littlewood identities proven in this paper correspond to decompositions of $(R, S)$ Macdonald polynomials (or $R$ Hall-Littlewood polynomials) indexed by rectangular partitions or half-partitions of maximal length. In the Schur case more general shapes have been considered in the literature. For example, Goulden and Krattenthaler [30,44,45] proved that the following formula for the character of the irreducible $\operatorname{Sp}(2 n, \mathbb{C})$-module of highest weight $\Lambda_{r}+(m-1) \Lambda_{n}$ :

$$
\sum_{\substack{\lambda \\ \lambda \leqslant 2 m \\ \operatorname{odd}(\lambda)=r}} s_{\lambda}(x)=\left(x_{1} \cdots x_{n}\right)^{m} \operatorname{sp}_{2 n,\left(m^{n-r}(m-1)^{r}\right)}(x),
$$

where $0 \leqslant r \leqslant n$ (and $\Lambda_{0}:=0$ ). For $r=0$ this is the Désarménien-ProctorStembridge formula (4.6).

Open problem 3. ( $i$ ) For positive integers $m, n$ and $r$ an integer such that $0 \leqslant r \leqslant n$, prove that

$$
\sum_{\substack{\lambda \\ \operatorname{odd}(\lambda)=r}} b_{\lambda ; m, r}^{\text {oa }}(q, t) P_{\lambda}(x ; q, t)=\left(x_{1} \cdots x_{n}\right)^{m} P_{m^{n-r}(m-1)^{r}}^{\left(\mathrm{C}_{n}, \mathrm{~B}_{n}\right)}(x ; q, t, q t),
$$

where

$$
b_{\lambda ; m, r}^{\mathrm{oa}}(q, t)=b_{\lambda}^{\mathrm{oa}}(q, t) \prod_{\substack{s \in \lambda / 1^{r} \\ a_{\lambda}^{\prime}(s) \text { even }}} \frac{1-q^{2 m-a_{\lambda}^{\prime}(s)} t_{\lambda}^{l_{\lambda}^{\prime}(s)}}{1-q^{2 m-a_{\lambda}^{\prime}(s)-1} t_{\lambda}^{\prime}(s)+1} .
$$

(ii) Prove similar such "non-rectangular" identities for other $(R, S)$. 
The map $\mathrm{e}^{-\alpha_{1}}, \ldots, \mathrm{e}^{-\alpha_{n}} \mapsto 1$ is known as the basic specialisation [61]. Applied to character formulas for affine Lie algebras leads to (generalised) Dyson-Macdonald type expansions for powers of the Dedekind eta-function, see e.g., [5, 24, 61, 69, 90, 98]. For example, taking the basic specialisation of $\mathrm{B}_{n}^{(1)}$ identity (5.18) (and replacing $t$ by $q$ ) yields the following generalisation of $[62$, p. $135,(6 \mathrm{c})]$ :

$$
\begin{aligned}
\frac{1}{\eta(\tau / 2)^{2 n} \eta(\tau)^{2 n^{2}-3 n}} \sum & (-1)^{\frac{|v|-|\rho|}{m+2 n-1}} \chi_{\mathrm{D}}(v / \rho) q^{\frac{\|v\|^{2}-\|\rho\|^{2}}{2(m+2 n-1)}+\frac{\|\rho\|^{2}}{2(2 n-1)}} \\
= & \sum_{\substack{\lambda \\
\lambda_{1} \leqslant m}} q^{|\lambda| / 2} P_{\lambda}^{\prime}(\underbrace{1, \ldots, 1}_{2 n-1 \text { times }} ; q) \prod_{i=0}^{m-1}\left(-q^{1 / 2} ; q^{1 / 2}\right)_{m_{i}(\lambda)} .
\end{aligned}
$$

Here $q=\exp (2 \pi \mathrm{i} \tau), \rho=(n-1, \ldots, 1,0)$,

$$
\chi_{\mathrm{D}}(v / w):=\prod_{i<j}\left(v_{i}^{2}-v_{j}^{2}\right) /\left(w_{i}^{2}-w_{j}^{2}\right),
$$

and the sum is over $v \in \mathbb{Z}^{n}$ such that $v_{i} \equiv \rho_{i}(\bmod m+2 n-1)$.

Let $C=C_{n}$ be the Cartan matrix of $\mathrm{A}_{n}$, i.e., $\left(C^{-1}\right)_{a b}=\min \{a, b\}-a b /(n+$ $1)$, and for $\left\{r_{i}^{(a)}\right\}_{1 \leqslant a \leqslant n ; 1 \leqslant i \leqslant k}$ a set of nonnegative integers, let

$$
R_{i}^{(a)}:=r_{i}^{(a)}+\cdots+r_{k}^{(a)} .
$$

Following [5, 98] we define

$$
F_{k, n}(q):= \begin{cases}\left(-q^{1 / 2} ; q\right)_{\infty}^{2 n-1} & \text { for } k=0 \\ \left(-q^{1 / 2} ; q^{1 / 2}\right)_{\infty}^{2 n-1} \sum_{\left\{r_{i}^{(a)}\right\}} \frac{q^{\frac{1}{2} \sum_{a, b=1}^{n} \sum_{i=1}^{k} C_{a b} R_{i}^{(a)} R_{i}^{(b)}}}{\prod_{a=1}^{n} \prod_{i=1}^{k-1}\left((q ; q)_{r_{i}^{(a)}}\right)\left(q^{2} ; q^{2}\right)_{r_{k}(a)}} & \text { for } k \geqslant 1\end{cases}
$$

and

$$
G_{k, n}(q):=(-q ; q)_{\infty}^{n} \sum_{\left\{r_{i}^{(a)}\right\}} \frac{q^{\frac{1}{2} \sum_{a, b=1}^{n} \sum_{i=1}^{k} C_{a b} R_{i}^{(a)} R_{i}^{(b)}\left(-q^{1 / 2-R_{1}^{(1)}} ; q\right)_{R_{1}^{(1)}}}}{\prod_{a=1}^{n} \prod_{i=1}^{k-1}\left((q ; q)_{r_{i}^{(a)}}\right)\left(q^{2} ; q^{2}\right)_{r_{k}^{(a)}}}
$$

for $k \geqslant 1$.

Open problem 4. For $m, n$ positive integers, prove that

$$
\begin{aligned}
\sum_{\substack{\lambda \\
\lambda_{1} \leqslant m}} q^{|\lambda| / 2} P_{\lambda}^{\prime}(\underbrace{1, \ldots, 1}_{2 n-1 \text { times }} ; q) \prod_{i=1}^{m-1}\left(-q^{1 / 2} ; q^{1 / 2}\right)_{m_{i}(\lambda)} & \\
& \stackrel{?}{=} \begin{cases}F_{k, 2 n-1}(q) & \text { if } m=2 k+1 \\
G_{k, 2 n-1}(q) & \text { if } m=2 k .\end{cases}
\end{aligned}
$$


For $n=1$ this follows from a minor modification of [96, Lemma A.1], for $m=1$ it follows from $P_{\left(1^{r}\right)}^{\prime}(x ; t)=e_{r}(x)$ and

$$
\sum_{r=0}^{\infty} z^{r} e_{r}\left[\frac{n}{1-q}\right]=(-z ; q)_{\infty}^{n},
$$

and for $m=2$ a proof is given in [5, Theorem 3.7]. By (6.1) the above problem for even $m$ is equivalent to [98, Conjecture 2.4; (2.6a)].

Most of the virtual Koornwinder integrals of Section 3 have elliptic analogues, see [79, 80].

Open problem 5. Prove elliptic analogues of the bounded Littlewood identities of Theorems 4.14 .6 .

For elliptic analogues of (4.1)-(4.3) we refer to [79].

\section{Appendix A. The Weyl-KaC Formula}

In this appendix we state some simple consequences of the Weyl-Kac formula, needed in the proofs of our combinatorial character formulas in Section 5.1 .

Recall the symplectic and odd-orthogonal Schur functions (2.60) and (2.62). It will be convenient to also define the normalised functions

(A.1) $\widetilde{\mathrm{sO}}_{2 n+1, \lambda}(x)=\Delta_{\mathrm{B}}(x) \mathrm{SO}_{2 n+1, \lambda}(x)$ and $\widetilde{\mathrm{sp}}_{2 n, \lambda}(x)=\Delta_{\mathrm{C}}(x) \mathrm{sO}_{2 n+1, \lambda}(x)$, so that $\widetilde{\operatorname{so}}_{2 n+1,0}(x)=\Delta_{\mathrm{B}}(x)$ and $\widetilde{\mathrm{sp}}_{2 n, 0}(x)=\Delta_{\mathrm{C}}(x)$.

Mimicking the proofs of [5. Lemmas 2.1-2.4] yields an expression for the characters of $\mathrm{B}_{n}^{(1)}$ and $\mathrm{A}_{2 n-1}^{(2)}$ in terms of the symplectic and odd orthogonal Schur functions as follows.

Lemma A.1 ( $\mathrm{B}_{n}^{(1)}$ character formula). Let

$$
x_{i}:=\mathrm{e}^{-\alpha_{i}-\cdots-\alpha_{n}} \quad(1 \leqslant i \leqslant n), \quad t:=\mathrm{e}^{-\delta},
$$

and parametrise $\Lambda \in P_{+}$, as

$$
\Lambda=c_{0} \Lambda_{0}+\left(\lambda_{1}-\lambda_{2}\right) \Lambda_{1}+\cdots+\left(\lambda_{n-1}-\lambda_{n}\right) \Lambda_{n-1}+2 \lambda_{n} \Lambda_{n},
$$

where $c_{0}$ is a nonnegative integer and $\lambda=\left(\lambda_{1}, \ldots, \lambda_{n}\right)$ a partition or halfpartition. Then

$$
\begin{gathered}
\mathrm{e}^{-\Lambda} \operatorname{ch} V(\Lambda)=\frac{1}{(t ; t)_{\infty}^{n} \prod_{i=1}^{n} \theta\left(x_{i} ; t\right) \prod_{1 \leqslant i<j \leqslant n} x_{j} \theta\left(x_{i} / x_{j}, x_{i} x_{j} ; t\right)} \\
\times \sum_{\substack{r \in \mathbb{Z}^{n} \\
|r| \equiv 0(2)}}{\widetilde{\mathrm{SO}_{2 n+1, \lambda}}}_{2\left(x t^{r}\right) \prod_{i=1}^{n} x_{i}^{\kappa r_{i}+\lambda_{i}} t^{\frac{1}{2} \kappa r_{i}^{2}-\left(n-\frac{1}{2}\right) r_{i}}}
\end{gathered}
$$


where $\kappa=2 n-1+c_{0}+\lambda_{1}+\lambda_{2}$.

Lemma A.2 $\left(\mathrm{A}_{2 n-1}^{(2)}\right.$ character formula). Let

$$
x_{i}:=\mathrm{e}^{-\alpha_{i}-\cdots-\alpha_{n-1}-\alpha_{n} / 2} \quad(1 \leqslant i \leqslant n), \quad t:=\mathrm{e}^{-\delta},
$$

and parametrise $\Lambda \in P_{+}$, as

$$
\Lambda=c_{0} \Lambda_{0}+\left(\lambda_{1}-\lambda_{2}\right) \Lambda_{1}+\cdots+\left(\lambda_{n-1}-\lambda_{n}\right) \Lambda_{n-1}+\lambda_{n} \Lambda_{n},
$$

where $c_{0}$ is a nonnegative integer and $\lambda=\left(\lambda_{1}, \ldots, \lambda_{n}\right)$ a partition. Then

$$
\begin{array}{r}
\mathrm{e}^{-\Lambda} \operatorname{ch} V(\Lambda)=\frac{1}{(t ; t)_{\infty}^{n-1}\left(t^{2} ; t^{2}\right)_{\infty} \prod_{i=1}^{n} \theta\left(x_{i}^{2} ; t^{2}\right) \prod_{1 \leqslant i<j \leqslant n} x_{j} \theta\left(x_{i} / x_{j}, x_{i} x_{j} ; t\right)} \\
\times \sum_{\substack{r \in \mathbb{Z}^{n} \\
|r| \equiv 0(2)}} \widetilde{\mathrm{sp}}_{2 n, \lambda}\left(x t^{r}\right) \prod_{i=1}^{n} x_{i}^{\kappa r_{i}+\lambda_{i}} t^{\frac{1}{2} \kappa r_{i}^{2}-n r_{i}},
\end{array}
$$

where $\kappa=2 n+c_{0}+\lambda_{1}+\lambda_{2}$.

Taking $\left(c_{0} ; \lambda\right)=\left(0 ;(m / 2)^{n}\right)$ in Lemma A.1 and $\left(0, m^{n}\right)$ in Lemma A.2 the above two formulas may be rewritten as a $D_{n}$-type sum over the full $\mathbb{Z}^{n}$ lattice.

Lemma A.3 $\left(\mathrm{B}_{n}^{(1) \dagger}\right.$ and $\mathrm{A}_{2 n-1}^{(2) \dagger}$ character formulas). Let

$$
x_{i}:=\mathrm{e}^{-\alpha_{i}-\cdots-\alpha_{n-1}+\left(\alpha_{n-1}-\alpha_{n}\right) / 2} \quad(1 \leqslant i \leqslant n), \quad t:=\mathrm{e}^{-\delta} .
$$

Then

$$
\begin{aligned}
& \mathrm{e}^{-m \Lambda_{0}} \operatorname{ch} V\left(m \Lambda_{0}\right)=\frac{1}{(t ; t)_{\infty}^{n} \prod_{i=1}^{n} \theta\left(t^{1 / 2} x_{i} ; t\right) \prod_{1 \leqslant i<j \leqslant n} x_{j} \theta\left(x_{i} / x_{j}, x_{i} x_{j} ; t\right)} \\
& \quad \times \sum_{r \in \mathbb{Z}^{n}} \Delta_{\mathrm{D}}\left(x t^{r}\right) \prod_{i=1}^{n}(-1)^{r_{i}} x_{i}^{\kappa r_{i}} t^{\frac{1}{2} \kappa r_{i}^{2}-(n-1) r_{i}}, \quad \kappa=m+2 n-1, \mathfrak{g}=\mathrm{B}_{n}^{(1) \dagger}
\end{aligned}
$$

and

$$
\begin{aligned}
& \mathrm{e}^{-m \Lambda_{0}} \operatorname{ch} V\left(m \Lambda_{0}\right) \\
& \quad=\frac{1}{(t ; t)_{\infty}^{n-1}\left(t^{2} ; t^{2}\right)_{\infty} \prod_{i=1}^{n} \theta\left(t x_{i}^{2} ; t^{2}\right) \prod_{1 \leqslant i<j \leqslant n} x_{j} \theta\left(x_{i} / x_{j}, x_{i} x_{j} ; t\right)} \\
& \times \sum_{r \in \mathbb{Z}^{n}} \Delta_{\mathrm{D}}\left(x t^{r}\right) \prod_{i=1}^{n}(-1)^{r_{i}} x_{i}^{\kappa r_{i}} t^{\frac{1}{2} \kappa r_{i}^{2}-(n-1) r_{i}}, \quad \kappa=2 m+2 n, \mathfrak{g}=\mathrm{A}_{2 n-1}^{(2) \dagger}
\end{aligned}
$$


For $m=0$ this gives what may be regarded as $\mathrm{B}_{n}^{(1) \dagger}$ and $\mathrm{A}_{2 n-1}^{(2) \dagger}$ Macdonald identities:

$$
\begin{aligned}
& \sum_{r \in \mathbb{Z}^{n}} \Delta_{\mathrm{D}}\left(x t^{r}\right) \prod_{i=1}^{n}(-1)^{r_{i}} x_{i}^{(2 n-1) r_{i}} t^{(2 n-1)}\left(\begin{array}{c}
r_{i} \\
2
\end{array}\right)+r_{i} / 2 \\
&=(t ; t)_{\infty}^{n} \prod_{i=1}^{n} \theta\left(t^{1 / 2} x_{i} ; t\right)_{\infty} \prod_{1 \leqslant i<j \leqslant n} x_{j} \theta\left(x_{i} / x_{j}, x_{i} x_{j} ; t\right)
\end{aligned}
$$

and

$$
\begin{aligned}
\sum_{r \in \mathbb{Z}^{n}} \Delta_{\mathrm{D}}\left(x t^{r}\right) \prod_{i=1}^{n}( & -1)^{r_{i}} x_{i}^{2 n r_{i}} t^{2 n\left(\begin{array}{c}
r_{i} \\
2
\end{array}\right)+r_{i}} \\
& =(t ; t)_{\infty}^{n-1}\left(t^{2} ; t^{2}\right)_{\infty} \prod_{i=1}^{n} \theta\left(t x_{i}^{2} ; t^{2}\right) \prod_{1 \leqslant i<j \leqslant n} x_{j} \theta\left(x_{i} / x_{j}, x_{i} x_{j} ; t\right) .
\end{aligned}
$$

Proof. We first consider the $\mathrm{B}_{n}^{(1) \dagger}$ case.

Setting $\left(c_{0} ; \lambda\right)=\left(0 ;(m / 2)^{n}\right)$ in (A.3) and using (2.62) and (A.1) yields the $\mathrm{B}_{n}^{(1)}$ character formula

$$
\begin{aligned}
& \mathrm{e}^{-m \Lambda_{n}} \operatorname{ch} V\left(m \Lambda_{n}\right)=\frac{1}{(t ; t)_{\infty}^{n} \prod_{i=1}^{n} \theta\left(x_{i} ; t\right) \prod_{1 \leqslant i<j \leqslant n} x_{j} \theta\left(x_{i} x_{j}, x_{i} / x_{j} ; t\right)} \\
& \times \sum_{\substack{r \in \mathbb{Z}^{n} \\
|r| \equiv 0(2)}} \operatorname{det}_{\substack{1 \leqslant i, j \leqslant n \\
\text { (n) }}}\left(x_{i}^{\kappa r_{i}+j-1} q^{\kappa\left(\begin{array}{c}
r_{i} \\
2
\end{array}\right)+(j-1) r_{i}}-x_{i}^{\kappa\left(r_{i}+1\right)-j+1} q^{\kappa\left(\begin{array}{c}
r_{i}+1 \\
2
\end{array}\right)-(j-1) r_{i}}\right),
\end{aligned}
$$

with $\kappa=m+2 n-1$ and $x_{1}, \ldots, x_{n}$ given by A.2. Replacing $\alpha_{i} \mapsto \alpha_{n-i}$ for $0 \leqslant i \leqslant n$ changes $\Lambda_{n}$ to $\Lambda_{0}$ on the left. On the right it has the effect of replacing (A.2) by

$$
x_{n-i+1}=\mathrm{e}^{-\alpha_{0}-\cdots-\alpha_{i-1}} \quad(1 \leqslant i \leqslant n) .
$$

Moreover, $t$ is now given by $t=\mathrm{e}^{-2 \alpha_{0}-\cdots-2 \alpha_{n-2}-\alpha_{n-1}-\alpha_{n}}$ in accordance with the interpretation of $\delta$ as the null root of $\mathrm{B}_{n}^{(1) \dagger}$ instead of $\mathrm{B}_{n}^{(1)}$.

Next we replace $x_{i} \mapsto t^{1 / 2} / x_{n+1-i}$ - So that $x_{i}$ is now given by (A.6) - and $r_{i} \mapsto r_{n+i-1}$ for $1 \leqslant i \leqslant n$. Also reversing the order of the rows and columns in the determinant and using $\theta(x ; t)=\theta(q / x ; t)$, we obtain

$$
\mathrm{e}^{-m \Lambda_{0}} \operatorname{ch} V\left(m \Lambda_{0}\right)=\frac{f_{0}}{(t ; t)_{\infty}^{n} \prod_{i=1}^{n} \theta\left(t^{1 / 2} x ; t\right) \prod_{1 \leqslant i<j \leqslant n} x_{j}\left(x_{i} x_{j}, x_{i} / x_{j} ; t\right)}
$$


where for $\sigma=0,1$

$$
\begin{aligned}
& f_{\sigma}:=\sum_{\substack{r \in \mathbb{Z}^{n} \\
|r| \equiv \sigma(2)}} \operatorname{det}_{1 \leqslant i, j \leqslant n}\left(x_{i}^{-\kappa r_{i}+j-1} t^{\frac{1}{2} \kappa r_{i}^{2}+(n-j) r_{i}}\right. \\
&\left.-x_{i}^{-\kappa\left(r_{i}+1\right)+2 n-j-1} t^{\frac{1}{2} \kappa\left(r_{i}+1\right)^{2}-(n-j)\left(r_{i}+1\right)}\right) .
\end{aligned}
$$

The claim now follows from the identity

$$
f_{\sigma}=(-1)^{\sigma} \sum_{r \in \mathbb{Z}^{n}} \Delta_{\mathrm{D}}\left(x q^{r}\right) \prod_{i=1}^{n}(-1)^{r_{i}} x_{i}^{\kappa r_{i}} q^{\frac{1}{2} \kappa r_{i}^{2}-(n-1) r_{i}} .
$$

To finally prove $(\underline{A .9}$ ) we proceed as follows:

$$
\begin{aligned}
& f_{0} \pm f_{1}= \sum_{r \in \mathbb{Z}^{n}} \operatorname{det}_{1 \leqslant i, j \leqslant n}\left((-1)^{\frac{1}{2}(1 \mp 1) r_{i}} t^{\frac{1}{2} \kappa r_{i}^{2}+(n-j) r_{i}} x_{i}^{-\kappa r_{i}+j-1}\right. \\
&\left.-(-1)^{\frac{1}{2}(1 \mp 1) r_{i}} t^{\frac{1}{2} \kappa\left(r_{i}+1\right)^{2}-(n-j)\left(r_{i}+1\right)} x_{i}^{-\kappa\left(r_{i}+1\right)+2 n-j-1}\right) \\
&= \operatorname{det}_{1 \leqslant i, j \leqslant n}\left(\sum_{r \in \mathbb{Z}}(-1)^{\frac{1}{2}(1 \mp 1) r} t^{\frac{1}{2} \kappa r^{2}+(n-j) r} x_{i}^{-\kappa r+j-1}\right. \\
&\left.-\sum_{r \in \mathbb{Z}}(-1)^{\frac{1}{2}(1 \mp 1) r} t^{\frac{1}{2} \kappa(r+1)^{2}-(n-j)(r+1)} x_{i}^{-\kappa(r+1)+2 n-j-1}\right) \\
&= \operatorname{det}_{1 \leqslant i, j \leqslant n}\left(\sum_{r \in \mathbb{Z}}(-1)^{\frac{1}{2}(1 \mp 1) r} t^{\frac{1}{2} \kappa r^{2}-(n-j) r} x_{i}^{\kappa r+j-1}\right) \\
&\left.\mp \sum_{r \in \mathbb{Z}}(-1)^{\frac{1}{2}(1 \mp 1) r} t^{\frac{1}{2} \kappa r^{2}+(n-j) r} x_{i}^{\kappa r+2 n-j-1}\right) \\
&=\sum_{r \in \mathbb{Z}^{n}} \operatorname{det}_{1 \leqslant i, j \leqslant n}\left((-1)^{\frac{1}{2}(1 \mp 1) r_{i}} t^{\frac{1}{2} \kappa r_{i}^{2}-(n-1) r_{i}} x_{i}^{\kappa r_{i}}\right. \\
&\left.\times\left(\left(x_{i} t^{r_{i}}\right)^{j-1} \mp\left(x_{i} t^{r_{i}}\right)^{2 n-j-1}\right)\right) .
\end{aligned}
$$

Here the second and last equality use multilinearity and the third equality follows from a shift of $r \mapsto r-1$ in the second sum over $r$. When $j=n$ the final line reads $(1 \mp 1)\left(x_{i} t^{r_{i}}\right)^{n-1}$, so that $f_{0}+f_{1}=0$. Moreover, by the $\mathrm{D}_{n}$ Vandermonde determinant (2.65),

$$
f_{0}-f_{1}=\frac{1}{2} \sum_{r \in \mathbb{Z}^{n}} \Delta_{\mathrm{D}}\left(x t^{r}\right) \prod_{i=1}^{n}(-1)^{r_{i}} t^{\frac{1}{2} \kappa r_{i}^{2}-(n-1) r_{i}} x_{i}^{\kappa r_{i}} .
$$

This completes the first part of our proof. 
The $\mathrm{A}_{2 n-1}^{(2)}$ case proceeds in almost identical fashion. This time we set take $\left(c_{0} ; \lambda\right)=\left(0 ; m^{n}\right)$ in (A.5). Using (2.60) and (A.1) yields the $\mathrm{A}_{2 n-1}^{(2)}$ character formula

$$
\begin{aligned}
\mathrm{e}^{-m \Lambda_{n}} \operatorname{ch} V\left(m \Lambda_{n}\right) & \frac{1}{(t ; t)_{\infty}^{n-1}\left(t^{2} ; t^{2}\right)_{\infty} \prod_{i=1}^{n} \theta\left(x_{i}^{2} ; t^{2}\right) \prod_{1 \leqslant i<j \leqslant n} x_{j} \theta\left(x_{i} / x_{j}, x_{i} x_{j} ; t\right)} \\
& \times \sum_{\substack{r \in \mathbb{Z}^{n} \\
|r| \equiv 0(2)}} \operatorname{det}_{1 \leqslant i, j \leqslant n}\left(x_{i}^{\kappa r_{i}+j-1} t^{\kappa\left(\begin{array}{c}
r_{i} \\
2
\end{array}\right)+(j-1) r_{i}}-x_{i}^{\kappa\left(r_{i}+1\right)-j+1} t^{\kappa\left(\begin{array}{c}
r_{i}+1 \\
2
\end{array}\right)-(j-1) r_{i}}\right)
\end{aligned}
$$

with $\kappa=2 m+2 n$ and $x_{1}, \ldots, x_{n}$ given by A.4. Noting that the summand is exactly the same as in (A.8) the rest of the proof follows mutatis mutandis.

\section{REFERENCES}

[1] G. E. Andrews, An analytic generalization of the Rogers-Ramanujan identities for odd moduli, Proc. Nat. Acad. Sci. USA 71 (1974), 4082-4085.

[2] G. E. Andrews, A. Schilling and S. O. Warnaar, An $A_{2}$ Bailey lemma and RogersRamanujan-type identities, J. Amer. Math. Soc. 12 (1999), 677-702.

[3] R. Askey and J. A. Wilson, Some basic hypergeometric polynomials that generalize Jacobi polynomials, Mem. Amer. Math. Soc. Vol. 319, AMS, Providence, RI, 1985.

[4] T. H. Baker and P. J. Forrester, Transformation formulas for multivariable basic hypergeometric series, Methods Appl. Anal. 6 (1999), 147-164.

[5] N. Bartlett and S. O. Warnaar, Hall-Littlewood polynomials and characters of affine Lie algebras, Adv. in Math., to appear; arXiv:1304.1602.

[6] A. Berkovich and S. O. Warnaar, Positivity preserving transformations for q-binomial coefficients, Trans. Amer. Math. Soc. 357 (2005), 2291-2351.

[7] D. Betea and M. Wheeler Refined Cauchy and Littlewood identities, plane partitions and symmetry classes of alternating sign matrices, arXiv:1402.0229.

[8] D. Betea, M. Wheeler and P. Zinn-Justin, Refined Cauchy/Littlewood identities and sixvertex model partition functions: II. Proofs and new conjectures, J. Algebraic Combin. to appear; arXiv:1405.7035.

[9] D. M. Bressoud, An analytic generalization of the Rogers-Ramanujan identities with interpretation, Quart. J. Math. Oxford Ser. (2) 31 (1980), 385-399.

[10] D. M. Bressoud, Analytic and combinatorial generalizations of the Rogers-Ramanujan identities. Mem. Amer. Math. Soc. Vol. 24, AMS, Providence, RI, 1980.

[11] D. M. Bressoud, Proofs and confirmations. The story of the alternating sign matrix conjecture, MAA Spectrum, Mathematical Association of America, Washington, DC, Cambridge University Press, Cambridge, 1999.

[12] D. M. Bressoud, Elementary proofs of identities for Schur functions and plane partitions, Ramanujan J. 4 (2000), 69-80.

[13] K. Bringmann, C. Calinescu, A. Folsom and S. Kimport, Graded dimensions of principal subspaces and modular Andrews-Gordon-type series, Commun. Contemp. Math. 16 (2014), 1350050, 20 pp. 
[14] S. A. Capparelli, A construction of the level 3 modules for the affine Lie algebra $A_{2}^{(2)}$ and a new combinatorial identity of the Rogers-Ramanujan type, Trans. Amer. Math. Soc. 348 (1996), 481-501.

[15] R. W. Carter, Lie Algebras of Finite and Affine Type, Cambridge Studies in Advanced Mathematics, 96, Cambridge University Press, Cambridge, 2005.

[16] I. Cherednik, Double Affine Hecke algebras, London Math. Soc. Lecture Note Series, 319, Cambridge University Press, Cambridge, 2005.

[17] I. Cherednik and B. Feigin, Rogers-Ramanujan type identities and Nil-DAHA, Adv. in Math. 248 (2013), 1050-1088.

[18] B. Coulson, S. Kanade, J. Lepowsky, R. McRae, F. Qi, M. C. Russell and C. Sadowski, A motivated proof of the Göllnitz-Gordon-Andrews identities, arXiv:1411.2044

[19] J. Désarménien, La démonstration des identités de Gordon et MacMahon et de deux identités nouvelles, Sém Lothar. Combin. B15a (1986), 11pp.

[20] J. Désarménien, Une généralisation des formules de Gordon et de MacMahon, C. R. Acad. Sci. Paris Sér. I Math. 309 (1989), 269-272.

[21] J. Désarménien, B. Leclerc and J.-Y. Thibon, Hall-Littlewood functions and KostkaFoulkes polynomials in representation theory, Sém. Lothar. Combin. 32 (1994), Art. B32c.

[22] J. F. van Diejen, Commuting difference operators with polynomial eigenfunctions, Compositio Math. 95 (1995), 183-233.

[23] J. F. van Diejen, Self-dual Koornwinder-Macdonald polynomials, Invent. Math. 126 (1996), 319-339.

[24] F. Dyson, Missed opportunities, Bull. Amer. Math. Soc. 78 (1972), 635-652.

[25] B. Feigin and E. Frenkel, Coinvariants of nilpotent subalgebras of the Virasoro algebra and partition identities, in I. M. Gel'fand Seminar, pp. 139-148, Adv. Soviet Math., 16, Part 1, AMS, Providence, RI, 1993.

[26] A. Feingold and A. Milas, The 3-state Potts model and Rogers-Ramanujan series, Cent. Eur. J. Math. 11 (2013), 1-16.

[27] G. Gasper and M. Rahman, Basic Hypergeometric Series, second edition, Encyclopedia of Mathematics and its Applications, Vol. 96, Cambridge University Press, Cambridge, 2004.

[28] B. Gordon, A combinatorial generalization of the Rogers-Ramanujan identities, Amer. J. Math. 83 (1961), 393-399.

[29] B. Gordon, Notes on plane partitions. V, J. Combin. Theory B 11 (1971), 157-168.

[30] I. P. Goulden, A linear operator for symmetric functions and tableaux in a strip with given trace, Discrete Math. 99 (1992), 69-77.

[31] M. J. Griffin, K. Ono and S.O. Warnaar, A framework of Rogers-Ramanujan identities and their arithmetic properties, Duke Math. J., to appear; arXiv:1401.7718.

[32] R. A. Gustafson, A generalization of Selberg's beta integral, Bull. Amer. Math. Soc. (N.S.) 22 (1990), 97-105.

[33] J. Haglund, The q,t-Catalan Numbers and the Space of Diagonal Harmonics, University lecture series, Vol. 41, AMS, Providence, RI, 2008.

[34] M. Ishikawa, F. Jouhet, J. Zeng, A generalization of Kawanaka's identity for HallLittlewood polynomials and applications, J. Algebraic Combin. 23 (2006), 395-412.

[35] M. Ishikawa and M. Wakayama, Applications of minor-summation formula. II. Pfaffians and Schur polynomials, J. Combin. Theory Ser. A 88 (1999), 136-157.

[36] V. K. Jain, Some transformations of basic hypergeometric functions. II. SIAM J. Math. Anal. 12 (1981), 957-961. 
[37] F. Jouhet and J. Zeng, New identities for Hall-Littlewood polynomials and applications, Ramanujan J. 10 (2005), 89-112.

[38] S. Kanade, J. Lepowsky, M. C. Russell and Andrew V. Sills, Ghost series and a motivated proof of the Andrews-Bressoud identities, arXiv:1411.2048

[39] J. Kaneko, q-Selberg integrals and Macdonald polynomials, Ann. Sci. École Norm. Sup. (4) 29 (1996), 583-637.

[40] J. Kaneko, Constant term identities of Forrester-Zeilberger-Cooper, Discrete Math. 173 (1997), 79-90.

[41] N. Kawanaka, On subfield symmetric spaces over a finite field, Osaka J. Math. 28 (1991), 759-791.

[42] N. Kawanaka, A q-series identity involving Schur functions and related topics, Osaka J. Math. 36 (1999), 157-176.

[43] T. H. Koornwinder, Askey-Wilson polynomials for root systems of type BC in Hypergeometric Functions on Domains of Positivity, Jack Polynomials, and Applications, pp. 189-204, Contemp. Math. 138, Amer. Math. Soc., Providence, 1992.

[44] C. Krattenthaler, Non-crossing two-rowed arrays and summations for Schur functions, in Proceedings of the 5th Conference on Formal Power Series and Algebraic Combinatorics, Florence, 1993, A. Barlotti, M. Delest, R. Pinzani, Eds., D.S.I., Università di Firenze, pp. 301-314.

[45] C. Krattenthaler, Identities for classical group characters of nearly rectangular shape, J. Algebra 209 (1998), 1-64.

[46] C. Krattenthaler, Plane partitions in the work of Richard Stanley and his school, arXiv:1503.05934

[47] R. Langer, M. J. Schlosser and S. O. Warnaar, Theta functions, elliptic hypergeometric series, and Kawanaka's Macdonald polynomial conjecture, SIGMA Symmetry Integrability Geom. Methods Appl. 5 (2009), paper 055, 20 pp.

[48] A. Lascoux, Symmetric Functions and Combinatorial Operators on Polynomials, CBMS Regional Conference Series in Mathematics Vol. 99, AMS, Providence, RI, 2003.

[49] A. Lascoux and M.-P. Schützenberger, Sur une conjecture de H. O. Foulkes, C. R. Acad. Sci. Paris Sér. A-B 286 (1978), A323-A324.

[50] A. Lascoux and S. O. Warnaar, Branching rules for symmetric functions and $\mathfrak{s l}_{n}$ basic hypergeometric series, Adv. in Appl. Math. 46 (2011), 424-456.

[51] J. Lepowsky, Generalized Verma modules, loop space cohomology and Macdonald-type identities, Ann. Sci. Ecole Norm. Sup. 12 (1979), 169-234.

[52] J. Lepowsky, Affine Lie algebras and combinatorial identities in Lie algebras and related topics, pp. 130-156, Lecture Notes in Mathematics No. 933, Springer, Berlin-New York, 1982.

[53] J. Lepowsky and S. Milne, Lie algebras and classical partition identities, Proc. Nat. Acad. Sci. U.S.A. 75 (1978), 578-579.

[54] J. Lepowsky and S. Milne, Lie algebraic approaches to classical partition identities, Adv. in Math. 29 (1978), 15-59.

[55] J. Lepowsky and R. L. Wilson, The Rogers-Ramanujan identities: Lie theoretic interpretation and proof, Proc. Nat. Acad. Sci. U.S.A. 78 (1981), 699-701.

[56] J. Lepowsky and R. L. Wilson, A new family of algebras underlying the RogersRamanujan identities and generalizations, Proc. Nat. Acad. Sci. U.S.A. 78 (1981), $7254-7258$.

[57] J. Lepowsky and R. L. Wilson, A Lie theoretic interpretation and proof of the RogersRamanujan identities, Adv. in Math. 45 (1982), 21-72. 
[58] J. Lepowsky and R. L. Wilson, The structure of standard modules. I. Universal algebras and the Rogers-Ramanujan identities, Inv. Math. 77 (1984), 199-290.

[59] D. E. Littlewood, The Theory of Group Characters and Matrix Representations of Groups, Oxford University Press, 1950.

[60] V. G. Kac, Infinite-dimensional algebras, Dedekind's $\eta$-function, classical Möbius function and the very strange formula, Adv. in Math. 30 (1978), 85-136.

[61] V. G. Kac, Infinite-dimensional Lie Algebras, 3rd Edition, Cambridge University Press, Cambridge, 1990.

[62] I. G. Macdonald, Affine root systems and Dedekind's $\eta$-function, Invent. Math. 15 (1972), 91-143.

[63] I. G. Macdonald, Symmetric functions and Hall polynomials, The Clarendon Press, Oxford University Press, New York, 1979.

[64] I. G. Macdonald, Symmetric functions and Hall polynomials, second edition, The Clarendon Press, Oxford University Press, New York, 1995.

[65] I. G. Macdonald, Orthogonal polynomials associated with root systems, Sém. Lothar. Combin. 45 (2000/01), Art. B45a, 40 pp.

[66] I. G. Macdonald, Affine Hecke Algebras and Orthogonal Polynomials, Cambridge Tracts in Mathematics, 157, Cambridge University Press, Cambridge, 2003.

[67] I. G. Macdonald, Hypergeometric Functions II (q-analogues), arXiv:1309.5208.

[68] Partitions of numbers whose graphs possess symmetry, Trans. Cambridge Phil. Soc. 17 (1898-99), 149-170.

[69] A. Milas, Virasoro algebra, Dedekind $\eta$-function and specialized Macdonald's identities, Transform. Groups 9 (2004), 273-288.

[70] K. Mimachi, A duality of Macdonald-Koornwinder polynomials and its application to integral representations, Duke Math. J. 107 (2001), 265-281.

[71] A. Meurman and M. Primc, Annihilating ideals of standard modules of $\operatorname{sl}(2, \mathbb{C}) \sim$ and combinatorial identities, Adv. in Math. 64 (1987), 177-240.

[72] S. Okada, Applications of minor summation formulas to rectangular-shaped representations of classical groups, J. Algebra 205 (1998), 337-367.

[73] A. Okounkov, BC-type interpolation Macdonald polynomials and binomial formula for Koornwinder polynomials, Transform. Groups 3 (1998), 181-207.

[74] A. Okounkov and N. Reshetikhin, Correlation function of Schur process with application to local geometry of a random 3-dimensional Young diagram, J. Amer. Math. Soc. 16 (2003), 581-603.

[75] G. Panova, Lozenge tilings with free boundaries, arXiv:1408.0417.

[76] R. A. Proctor, New symmetric plane partition identities from invariant theory work of De Concini and Procesi, European J. Combin. 11 (1990), 289-300.

[77] E. M. Rains, $B C_{n}$-symmetric polynomials, Transform. Groups 10 (2005), 63-132.

[78] E. M. Rains, $B C_{n}$-symmetric abelian functions, Duke Math. J. 135 (2006), 99-180.

[79] E. M. Rains, Elliptic Littlewood identities, J. Combin. Theory Ser. A 119 (2012), 15581609.

[80] E. M. Rains, Multivariate quadratic transformations and the interpolation kernel, arXiv:1408.0305.

[81] E. M. Rains and M. Vazirani, Vanishing integrals of Macdonald and Koornwinder polynomials, Transform. Groups 12 (2007), 725-759.

[82] L. J. Rogers, Second memoir on the expansion of certain infinite products, Proc. London Math. Soc. 25 (1894), 318-343. 
[83] H. Rosengren, Selberg integrals, Askey-Wilson polynomials and lozenge tilings of a hexagon with a triangular hole, arXiv:1503.00971.

[84] H. Rosengren and M. Schlosser, Elliptic determinant evaluations and the Macdonald identities for affine root systems, Compos. Math. 142 (2006), 937-961.

[85] S. Sahi, Nonsymmetric Koornwinder polynomials and duality, Ann. of Math. (2) 150 (1999), 267-282.

[86] I. Schur, Aufgabe 569, Arch. Math. Phys. 27 (3) (1918), 163; Ges. Abhandlungen, Vol. 3, p. 456.

[87] L. J. Slater, Further identities of the Rogers-Ramanujan type, Proc. London Math. Soc. (2) 54 (1952), 147-16.

[88] J. R. Stembridge, Hall-Littlewood functions, plane partitions, and the RogersRamanujan identities, Trans. Amer. Math. Soc. 319 (1990), 469-498.

[89] J. V. Stokman, Macdonald-Koornwinder polynomials, arXiv:1111.6112

[90] A. V. Stoyanovsky, Lie algebra deformations and character formulas, Funct. Anal. Appl. 32 (1998), 66-68.

[91] G. Szegö, Beitrag zur theorie der thetafunktionen, Sitz Preuss. Akad. Wiss. Phys. Math. Ki. XIX, (1926), 242-252.

[92] V. Venkateswaran, Vanishing integrals for Hall-Littlewood polynomials, Transform. Groups 17 (2012), 259-302.

[93] V. Venkateswaran, Symmetric and nonsymmetric Hall-Littlewood polynomials of type $B C$, arXiv:1209.2933.

[94] S. O. Warnaar, The generalized Borwein conjecture. II. Refined q-trinomial coefficients, Discrete Math. 272 (2003), 215-258.

[95] S. O. Warnaar, $q$-Selberg integrals and Macdonald polynomials, Ramanujan J. 10 (2005), 237-268.

[96] S. O. Warnaar, Rogers-Szegö polynomials and Hall-Littlewood symmetric functions, J. Algebra 303 (2006), 810-830.

[97] S. O. Warnaar, Bisymmetric functions, Macdonald polynomials and $\mathfrak{s l}_{3}$ basic hypergeometric series, Compositio Math. 114 (2008), 271-303.

[98] S. O. Warnaar and W. Zudilin, Dedekind's eta-function and Rogers-Ramanujan identities, Bull. London Math. Soc. 44 (2012), 1-11.

Department of Mathematics, California Institute of Technology, Pasadena, CA 91125, USA

E-mail address: rains@caltech.edu

School of Mathematics and Physics, The University of Queensland, BrisBAne, QLD 4072, Australia

E-mail address: o.warnaar@maths.uq.edu.au 